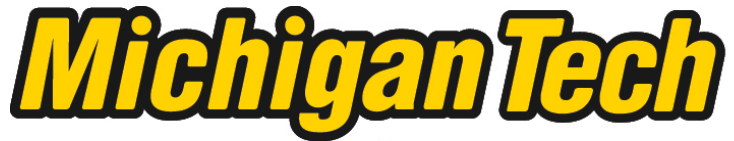 \\ Michigan Technological University Create the Future Digital Commons @ Michigan Tech
}

Dissertations, Master's Theses and Master's Reports - Open

Dissertations, Master's Theses and Master's

Reports

2010

\section{Vibro-acoustical analysis and design of a multiple-layer constrained viscoelastic damping structure}

Fei Lin

Michigan Technological University

Follow this and additional works at: https://digitalcommons.mtu.edu/etds

Part of the Mechanical Engineering Commons

Copyright 2010 Fei Lin

\section{Recommended Citation}

Lin, Fei, "Vibro-acoustical analysis and design of a multiple-layer constrained viscoelastic damping structure", Dissertation, Michigan Technological University, 2010.

https://doi.org/10.37099/mtu.dc.etds/383

Follow this and additional works at: https://digitalcommons.mtu.edu/etds

Part of the Mechanical Engineering Commons 


\title{
VIBRO-ACOUSTICAL ANALYSIS AND DESIGN OF A MULTIPLE-LAYER CONSTRAINED VISCOELASTIC DAMPING STRUCTURE
}

By

Fei Lin

\author{
A DISSERTATION \\ Submitted in partial fulfillment \\ of the requirements for the degree of \\ DOCTOR OF PHILOSOPHY \\ in
}

Mechanical Engineering-Engineering Mechanics

MICHIGAN TECHNOLOGICAL UNIVERSITY

2010

Copyright (C) Fei Lin 2010 
This dissertation, "Vibro-acoustical Analysis and Design of a Multiple-Layer Constrained Viscoelastic Damping Structure", is hereby approved in partial fulfillment of the requirements for the Degree of DOCTOR OF PHILOSOPHY in Mechanical Engineering-Engineering Mechanics.

\section{DEPARTMENT:}

Mechanical Engineering-Engineering Mechanics

\begin{tabular}{|c|c|}
\hline \multicolumn{2}{|l|}{ Dissertation Advisor } \\
\hline \multirow{3}{*}{ Department Chair } & Dr. Mohan D. Rao \\
\hline & \\
\hline & Dr. William W. Predebon \\
\hline Date & \\
\hline
\end{tabular}




\section{Abstract}

The goal of this research is to provide a framework for vibro-acoustical analysis and design of a multiple-layer constrained damping structure. The existing research on damping and viscoelastic damping mechanism is limited to the following four mainstream approaches: modeling techniques of damping treatments/materials; control through the electrical-mechanical effect using the piezoelectric layer; optimization by adjusting the parameters of the structure to meet the design requirements; and identification of the damping material's properties through the response of the structure. This research proposes a systematic design methodology for the multiple-layer constrained damping beam giving consideration to vibro-acoustics.

A modeling technique to study the vibro-acoustics of multiple-layered viscoelastic laminated beams using the Biot damping model is presented using a hybrid numerical model. The boundary element method (BEM) is used to model the acoustical cavity whereas the Finite Element Method (FEM) is the basis for vibration analysis of the multiple-layered beam structure. Through the proposed 
procedure, the analysis can easily be extended to other complex geometry with arbitrary boundary conditions. The nonlinear behavior of viscoelastic damping materials is represented by the Biot damping model taking into account the effects of frequency, temperature and different damping materials for individual layers. A curve-fitting procedure used to obtain the Biot constants for different damping materials for each temperature is explained. The results from structural vibration analysis for selected beams agree with published closed-form results and results for the radiated noise for a sample beam structure obtained using a commercial BEM software is compared with the acoustical results of the same beam with using the Biot damping model. The extension of the Biot damping model is demonstrated to study MDOF (Multiple Degrees of Freedom) dynamics equations of a discrete system in order to introduce different types of viscoelastic damping materials.

The mechanical properties of viscoelastic damping materials such as shear modulus and loss factor change with respect to different ambient temperatures and frequencies. The application of multiple-layer treatment increases the damping characteristic of the structure significantly and thus helps to attenuate the vibration and noise for a broad range of frequency and temperature. 
The main contributions of this dissertation include the following three major tasks:

1) Study of the viscoelastic damping mechanism and the dynamics equation of a multilayer damped system incorporating the Biot damping model.

2) Building the Finite Element Method (FEM) model of the multiple-layer constrained viscoelastic damping beam and conducting the vibration analysis.

3) Extending the vibration problem to the Boundary Element Method (BEM) based acoustical problem and comparing the results with commercial simulation software. 


\section{Acknowledgement}

I would like to extend my thanks to Dr. Mohan Rao of Michigan Tech for his guidance, support, and encouragement through the years of my $\mathrm{PhD}$ study, research and help with the writing of my dissertation. Many thanks to the committee members: Dr. Spandan Maiti, Dr. Bo Chen and Dr. Zhanping You for their time and participation in both the research proposal and the final defense.

I also would like to express gratitude to Dr. Kaiyu Xu, Professor of Shanghai University for helpful discussion on the FE modeling of multiple-layered sandwich beam and his encouragement on this research. Thanks to the colleagues and friends at Michigan Tech for friendship and help in many aspects. Finally, I want to appreciate my wife, Ye Wang whose love and support are immeasurable, and my parents, Bing Peng and Shuangdong Lin, to whom I owe the most, for their continuous love and support through the years. 


\section{Index of Contents}

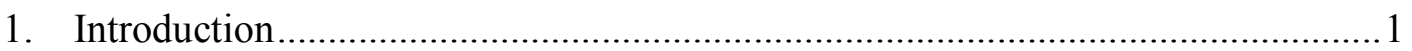

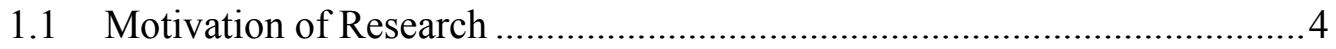

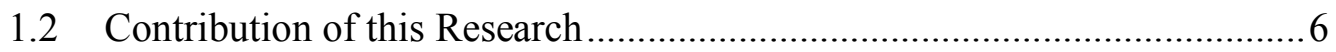

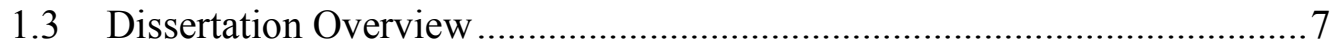

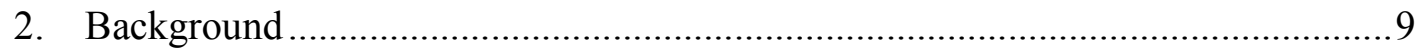

2.1 Early Research of Damping Treatments....................................................

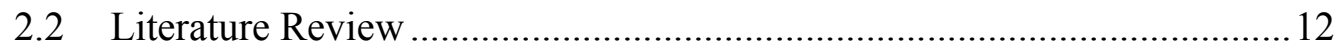

2.1.1 Analytical Modeling of Constrained-Layer Treatment.....................12

2.1.2 Numerical Implementation of Constrained-Layer Treatment..........15

2.1.3 Modern Damping Models ................................................................. 18

2.1.4 Control Strategies.....................................................................23

2.1.5 Damping Identification and Optimization ………............................26

2.3 Concepts and Properties of Viscoelastic Material........................................30

2.3.1 Temperature-Frequency Dependent Properties ………………….....30

2.3.2 Damping Mechanism .........................................................................

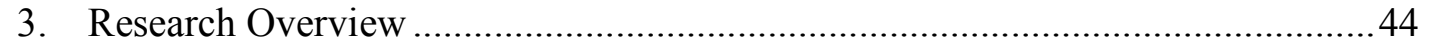

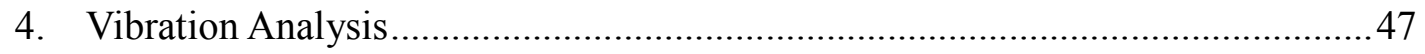


4.1 FE Modeling of the Multiple-Layer Beam...........................................4 47

4.1.1 Two Fundamental Components in a Multiple-Layer Beam............47

4.1.2 FE Modeling of a Seven-Layer Constrained Damping Beam .........54

4.2 Derivation of the Biot Dynamic Equation..............................................58

4.3 Decoupling Transformation and Dynamic Solution................................66

4.4 Dimension Reduction Technique ................................................. 72

4.5 Parametric Determination of the Biot Damping Model ............................76

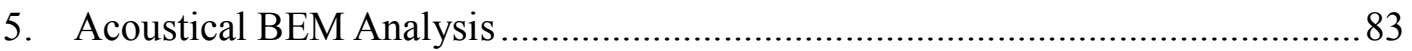

5.1 Introduction of Acoustical BEM Theory ............................................ 83

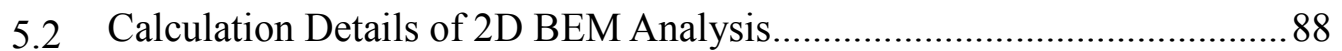

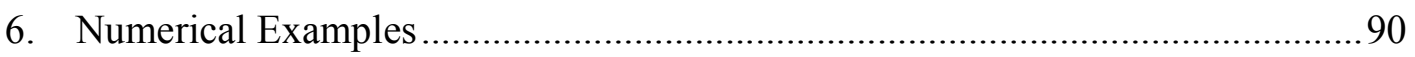

6.1 Example of a 2 DOF Lumped-Mass Model .........................................90

6.2 Example of a Three-Layer Sandwich Beam...........................................95

6.3 Vibro-Acoustic Response Example of a Seven-Layer Beam .....................99

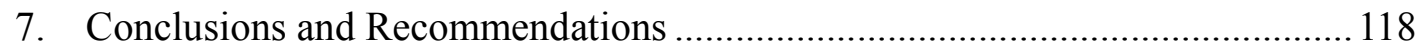

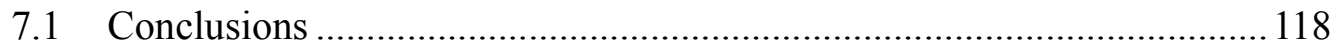

7.2 Recommendation for Future Research ................................................ 120

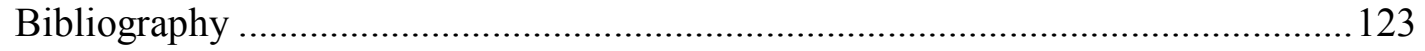

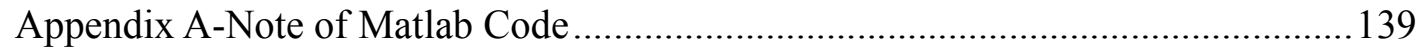


Appendix B-Curve fitting Datasheet for 3M-ISD110/112 .................................... 140

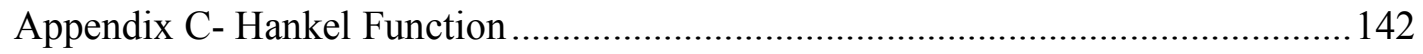




\section{List of Figures}

Figure 2.1 Typical Free-Layer Damping Treatment........................................ 10

Figure 2.2 Typical Constrained-Layer Damping Treatment ..............................11

Figure 2.3 Variation of shear modulus of a viscoelastic material with varying

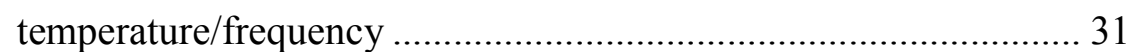

Figure 2.4 Relaxation plot in different regions ............................................ 32

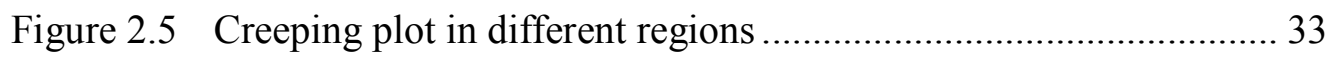

Figure 2.6 Mechanical analogue on series of Maxwell elements...................... 38

Figure 2.7 Relationships of different damping models .................................... 43

Figure 3.1 Schematic diagram of the semi-coupled problem............................ 44

Figure 3.2 Flow chart of the key steps in this research ................................... 46

Figure 4.1 Configuration of the elastic layer showing the DOF …………...... 47

Figure 4.2 Configuration of the constrained damping layer showing the DOF49

Figure 4.3 Deformation relationship of the viscoelastic core ………….......... 52

Figure 4.4 Configuration of a seven-layer damping structure showing the DOF 55

Figure 4.5 Comparison of the matrix dimension with/without the dimension 


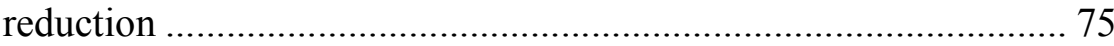

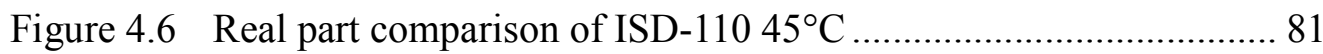

Figure 4.7 Imaginary part comparison of ISD-110 $45^{\circ} \mathrm{C} \ldots \ldots \ldots \ldots \ldots \ldots \ldots \ldots \ldots . . . \ldots 1$

Figure 4.8 Relative error percentage comparison of ISD- $11045^{\circ} \mathrm{C}$.............. 82

Figure 5.1 Notations of 2D BEM interior problem in fluid domain V ........... 84

Figure 5.2 Layout of BEM acoustical cavity problem ................................ 89

Figure 6.1 Schematic diagram of a lumped mass system- case1 .................. 90

Figure 6.2 System displacements under the unit step excitation on mass\#1 .... 91

Figure 6.3 Schematic diagram of a lumped-mass system- case 2 .................. 92

Figure 6.4 System displacements on the unit step excitation on mass \#1 1........ 93

Figure 6.5 Schematic diagram of 3-layer damping structure ......................... 96

Figure 6.6 Seven-layer sandwich structure with viscoelastic cores ................ 99

Figure $6.7 \mathrm{a}-\mathrm{b} 1^{\text {st }}$ order system damped frequency/loss factor

(simply-supported B.C.) .................................................. 103

Figure 6.8a-b First damped frequency and loss factor ............................. 105

Figure 6.9 System displacements under the unit step excitation.................. 106

Figure 6.10 Transverse velocity under the impulse excitation (at middle node \#7) 107

Figure 6.11 Contour plot of SPL when the impulse force applied $(10 \mathrm{~Hz}) \ldots \ldots .108$ 
Figure 6.12 Element result of sound pressure level $(10 \mathrm{~Hz})$ 109

Figure 6.13 Nodal frequency spectrum on $(0.5,0.4 \mathrm{~m})$ .110

Figure 6.14 Element layout of 3D seven-layer damping beam 111

Figure 6.15 Acoustical BEM analysis in LMS Virtual.Lab .114

Figure 6.16 SPL comparision between VL and 2D BEM calculation at field point $(0.5,0.4)$ 115 


\section{List of Tables}

Table 2.1 Explanation of Maxwell and Kelvin damping model ......................35

Table 2.2 Proposed frequency-domain damping models ..............................42

Table 4.1 Arrhenius coefficients from ref [94] …......................................79

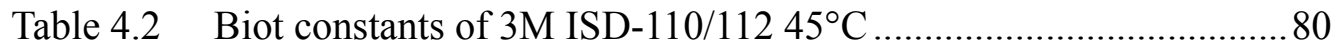

Table 6.1 Parameters comparison of lumped-mass cases .............................94

Table 6.2 Biot parameters of $3 \mathrm{M}$ ISD- $11045^{\circ} \mathrm{C}$..........................................95

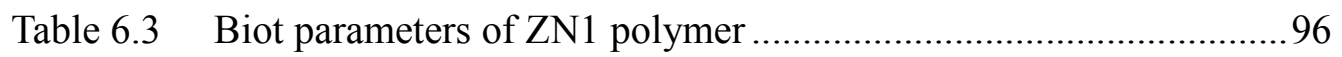

Table 6.4 Design parameters of three-layer structure ..................................96

Table 6.5 Comparison between the results from simulation and references

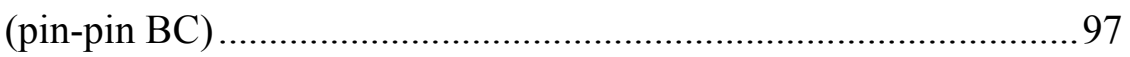

Table 6.6 Comparison between the results from simulation and references

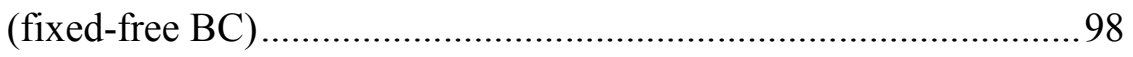

Table 6.7 Design parameters of seven-layer structure ................................99

Table 6.8 Comparison of results for simply supported boundary condition.. 101

Table 6.9 Comparison of results for fixed-free boundary condition.............. 101

Table 6.10 Comparison of modal results with ANSYS simulations................ 112

Table 6.11 1st system damped frequency/loss factor/SPL (simply-supported

B.C.) 


\section{Nomenclature}

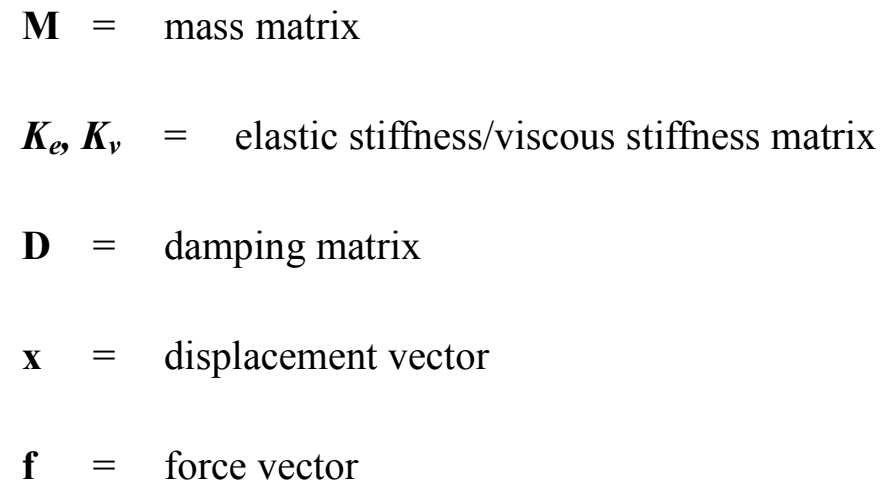

$\mathbf{A}, \mathbf{B}=$ coefficient matrix of state equation

$\mathbf{z}=$ dissipation coordinate vector

$\mathbf{m}, \mathbf{n}=$ number of mini-oscillators for $1^{\text {st }} / 2^{\text {nd }}$ type of viscoelastic material

$\mathbf{s}=$ Laplace variable

$\mathbf{t}=$ time

$\mathbf{E}=$ Young's Modulus

$\mathbf{G}=$ Shear Modulus

$\mathbf{N}_{\mathbf{e}}, \mathbf{N}_{\mathbf{f}}=$ FE Shape function of longitudinal/transverse deflection

$\mathbf{N}=$ number of DOFs

$\boldsymbol{\rho}=$ density of material

$\mathbf{h}=$ thickness of layer 
$1=$ length of beam

$\boldsymbol{\Phi}=$ eigenvector matrix

$\lambda=$ eigenvalue matrix

$G^{\infty},\left\{\mathrm{a}_{\mathrm{k}}, \mathrm{b}_{\mathrm{k}}\right\}=$ Biot constants

$\mathbf{p}=$ sound pressure

$\mathbf{v}=$ velocity vector

$\mathbf{v}_{\mathbf{n}}=$ nodal normal component of boundary velocity 


\section{Introduction}

Nowadays, addressing vibration and noise issues is essential to the improvement of performance and operational perception in advanced engineering structures and systems. Passive and active structural damping can attenuate a system's vibration and noise through the proper use of materials that posses enhanced damping properties.

In recent research, the most popular method to make this attenuation more predictable has been the use of material damping; this typically involves the application of high-damping materials like viscoelastic materials. For almost half of a century, researchers have conducted studies on topics including: analytical or numerical modeling techniques of different damping treatments; mathematical representations of damping properties; control strategies by the piezoelectric material and optimization or identification of the damping structure. Among the different viscoelastic damping treatments, constrained-layer damping structures are the most efficient approach when introducing damping to a system.

Many studies focus on the constrained-layered viscoelastic structure. The majority 
of these studies are based on the three-layer constrained sandwich beam due to its ability to include all of the factors that influence the system damping properties. It turns out that relatively few works have focused on the multiple-layer constrained sandwich beam and its ability to further reduce noise and vibration. Further, the acoustical performance of the damping structure is increasingly focused on the arbitrary type of excitation. Hence, it is of great importance to study the vibration and acoustical performance of the multiple-layer sandwich beam as it relates to the changes of influencing factors. The objective of my research is to propose a systematic vibro-acoustical design for the multiple-layer constrained damping beam and to establish a quantitative relationship between vibro-acoustical responses and external factors, including ambient temperature, frequency, combinations of different materials, excitation type, etc.

This research began with an in depth investigation into the damping mechanism using the frequency-domain Biot model. In order to study the vibration characteristics of the damping system, using this Biot damping model, several numerical examples were studied including the lumped-mass system and the multiple-layer sandwich beam modeled by the Finite Element (FE) technique. The semi-coupled acoustical problem was solved by the Boundary Element (BE) 
technique. These investigations and the resulting calculations are the major contributions of this research in damping. The background is the result of a detailed literature review and provides a concise introduction to recent damping mechanism theory. 


\subsection{Motivation of Research}

In order to stay competitive in the contemporary marketplace, manufacturers of advanced engineering products need to place a strong emphasis on their consumers' level of comfort when using their product. The presence of excessive noise can greatly reduce a consumer's perception of comfort. Thus, there is an increasing demand for systematic research on the application of damping structures that can ensure a perception of comfort in the consumers. Any analysis of multiple-layer damping structures must take into consideration to both the vibration as well as the noise aspects of the problem.

Because many of the contributions in vibro-acoustical research have been made in the area of the single-layer damping structure, it can be concluded that there is a gap in the literature surrounding the areas of:

1. Numerical representation for a multiple-layer constrained damping beam using the Finite Element(FE) model, 
2. Developing the dynamic equation with the Biot damping model for different viscoelastic materials, calculating the vibration response analysis in the time domain as well frequency domain,

3. Finding a robust and efficient curve-fitting technique for the complex shear modulus at different ambient temperatures,

4. Extending the vibration problem to a semi-coupled vibro-acoustical analysis using acoustical BE technique for the multiple-layer constrained damping beam, and

5. Investigating the quantitative relationship between internal factors (such as vibro-acoustical responses and viscoelastic properties) and external factors. 


\subsection{Contribution of this Research}

The work presented in this dissertation is very useful in the design and analysis of viscoelastic damping structures. With the growing demand for vibration and acoustical attenuation techniques and tools, especially for structures incorporating different viscoelastic damping materials into the numerical geometry, the analyis technique presented in this dissertation is practical. Filling the research gap identified from literature review, this research contributes in the application of the Biot Dynamic Equation with different viscoelastic damping materials with the multiple-layered FE structure in addition to studies of the vibro-acoustical problem of the multiple-layered damping beam.

It is anticipated that this research will support the design or research activities for NVH (Noise, Vibration and Harshness) engineers and scientists as well as the researchers in related fields. In particular, the completion and results of this research can be considered as the starting point of an extensive study into the formulation of a more complicated damping structure associated with the vibro-acoustical analysis and the effect of many related factors, such as the ambient temperature, type of excitation, etc. 


\subsection{Dissertation Overview}

The following is a brief outline of the dissertation:

In Chapter 1, the research introduction, objectives, motivations and outlines are discussed. Chapter 2 details the important concepts which form the basis for viscoelastic damping, the different types of damping treatments, and the theory of damping mechanism. Chapter 3 will provide an illustrative explanation of this research.

Chapter 4 will present the vibration analysis of multiple-layer structure. It will also discuss the finite element model, the Biot damping dynamic model, the model-order reduction theory, the complex shear-modulus curve-fitting technique and decoupling transformation.

Chapter 5 introduces the direct boundary element method (DBEM) theory and extends the vibration problem in Chapter 4 to an acoustical cavity problem 
Chapter 6 will further illustrate the concepts through numerical examples. This chapter discusses the lumped mass model, and provides an example of the vibration of a three layer beam. It also discusses the vibro-acoustical responses of a seven layer beam to external factors such as ambient temperature, frequency, combination of different materials, etc.

Finally, Chapter 7 presents a summary of the proposed research and its role in the broader context of vibro-acoustical design. It also mentions the opportunities for further research for other irregular shaped structures containing viscoelastic damping materials. 


\section{Background}

\subsection{Early Research of Damping Treatments}

The early application of viscoelastic damping to facilitate the energy dissipation can be traced back to 1919 when Lord Corporation was looking for a rubber material bonded to metal sheet for noise and vibration reduction. The first publications are by Lienard [1] and Oberst [2] in early 1950s. In Lienard's publication, the mathematical model of "loss mechanism" is not studied. The application of this damping mechanism is applied to the thin automobile panels for the noise emission reduction. Oberst dealt with the different configuration of experiment on the single-homogenous layers of damping materials in his follow-up publications[2-4].

In the same era, researchers have studied two types of viscoelastic damping treatments: free-layer treatment proposed by Plass (1957) [5] and the constrained-layer treatment proposed by Kerwin (1959) [6] .

Free layer treatments (shown in Figure 2.1) studied by Plass [5] are widely used in 
the automotive industry as additional layers on large sheet metal panels. The elongation between the supporting metallic and the viscoelastic due to the bending of supporting plates in the low frequency range is the mechanism introducing the material damping. The disadvantage of this structure is: when carrying a significant load, the viscoelastic must thus be quite stiff. However, the high damping material which is very energy-dissipative cannot be utilized in this case.

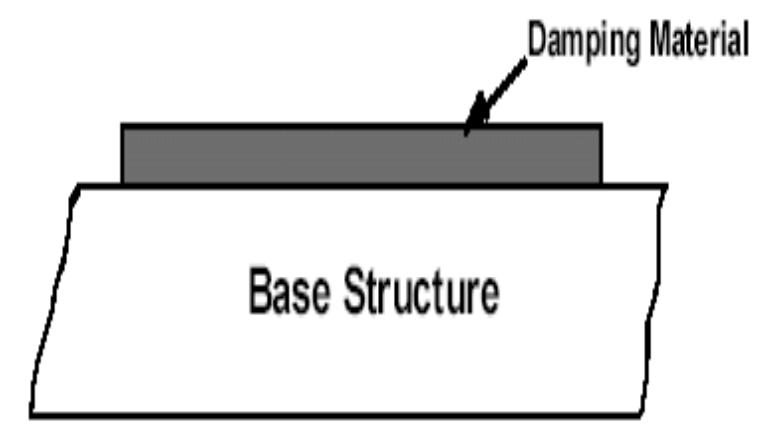

Figure 2.1 Typical Free-Layer Damping Treatment

Constrained layer treatments (shown in Figure 2.2) investigated by Kerwin [6] are an excellent option allowing high dissipative performance with soft viscoelastic materials. The mechanism that having a thin and soft viscoelastic layer transmitting shear loads between two stiff metal layers ensures the high dissipative performance of structure. Some follow-up publications $[7,8]$ by Kerwin et al. also made many efforts on different configurations of experiment comparing the free-layer and 
constrained-layer damping treatments. They concluded that the constrained-layer treatment is more weight-efficient in different testing configurations.

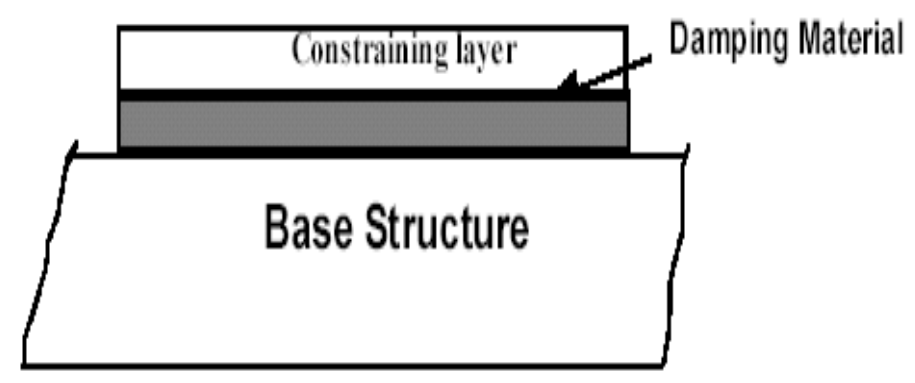

Figure 2.2 Typical Constrained-Layer Damping Treatment

Comparing these two types, the mechanism of constrained damping ensures higher damping (usually measured by loss factor) because of the energy dissipated during the shear deformation of the viscoelastic material due to the mismatch of base and constrained layers. 


\section{$2.2 \quad$ Literature Review}

Researches on the viscoelastic damping structure can be categorized on the basis of the following four mainstream topics:

1. Damping treatment and its numerical implementation

2. Damping model to represent the viscoelastic material's behavior

3. Control strategies

4. Damping Identification/ Optimization on the number of layers, sizing, placement, etc.

\subsubsection{Analytical Modeling of Constrained-Layer Treatment}

To mathematically model the constrained-layer damping beam, DiTaranto (1965) [9] developed six-order partial differential equation (PDE) in terms of axial displacements. In 1968, Mead and Markus [10] published the derivation of a six-order PDE for constrained-layer damping beam. Their derivation can be applied to mathematically express the transverse displacement for various boundary conditions. Although Kerwin published the initial assumption of modeling 
constrained-layer structure, this paper is considered as the classical closed-form solution. A paper by Rao [11] presented the equations of motion and boundary conditions using the energy method. The equations were solved numerically and a practical design guideline was presented. Similar to Rao's theory, Cottle [12] used Hamilton's principle to derive equations of motion.

There were hundreds of papers on the theory and application of constrained-layer damping treatment from 1950 s to early 1980 s, the era when numerical analysis (mostly Finite Element) are not economical comparing to the closed-form approximation. These publications are mostly utilizing Kerwin's model to make minor changes on individual's application, or making some changes to investigate Kerwin's assumption. Torvik (1980) [13], Mead (1982) [14] and Nakra [15-17] published the excellent reviews on the development of constrained layer damping treatment in this era. Among these reviews, Mead [14] concluded the basic assumption made by most researchers and his Mead and Markus [10] model was termed by Douglas and Yang (1978) [18].

In recent decades, researchers studied different methods to reduce the noise and vibration for the sandwich beam by increasing the number of layers. Ma [19] 
proposed an analytical equation for transverse vibration using Hamilton's principle and calculated the loss factor and natural frequency for a five-layer simply-supported sandwich beam with the complex shear modulus. The comparison with the computational result of three-layer and four-layer sandwich beam from the former research by other researcher, both experimental and analytical, was presented by Ma

Following with the Hamilton's principle, Hao and Rao [20] developed the closed-form solution for both symmetrical and asymmetrical simply-supported multiple layer sandwich beam, extending the number of layers from limited to unlimited. This paper also correlated the experimental results with the numerical example of a three-layer sandwich beam with two different viscoelastic layers adjacent to each other.

Teng and $\mathrm{Hu}$ [21] proposed the alternative analytical method of building the constrained layer damping structure by employing the RKU (Ross-Kervin-Ungar) [8] model. The possible factors such as frequency, temperature, sizing of damped structure that might affect vibration and noise attenuating performance are also discussed in this paper. 
The damping could also be increased by adding passive stand-off layer (PSOL) and slotted standoff layer (SSOL) to the layered systems. Falugi [22] and Parin [23] did theoretical and experimental work on a four-layered panel and a five-layered beam with PSOL treatment. Rogers and Parin [24], and Yellin, Shen and Reinhall [25] have performed experimental investigations and demonstrated that PSOL treatment increased the damping significantly in aeronautical structures and beams. Yellin and colleagues $[26,27]$ also developed normalized equations of motion for beam, fully treated with PSOL using non-ideal stand-off layer assumption. The equations were solved using the method of distributed transfer functions [28].

\subsubsection{Numerical Implementation of Constrained-Layer Treatment}

In 1980s, the concept of constrained-layer damping has wide applications in the following decades. Many applications can be found in the conference proceedings [29-33] sponsored by the Air Force Wright Aeronautical Laboratories between 1980s and 1990s. The engineering models that applicable to industrial problems is quite emergent in this background. The first publication utilizing FE method is by 
Johnson et al. [34] in 1981 on the basis of damping-energy relationship found by Ungar and Kerwin (1962) [35]. Johnson's work rests on the modal strain energy (MSE) method. This method has the dominant position for industrial application especially aerospace by combining complex FE geometry and MSE method.

In 1990s, many researchers turned research interest to the Finite Element Method (FEM) for building the numerical model of the constrained-layer system.

Lee and Lesieutre (1996) [36] proposed the 3 nodes, 10 DOF FE model for the three-layer Active Constrained Layer (ACL) damping beam. This FE model is advantageous in active control application due to its features of non-shear locking and adaptability of segmented constraining layers.

In 1999, Chen and Chan [37] studied four different types of integral FEM model with a viscoelastic core: 8 and 12 DOFs(Degrees of Freedom) of sandwich beam elements; 33 and 42 DOFs of sandwich plate/shell elements. The numerical stability, accuracy as well as convergence issue of these four different FEM models are demonstrated through the comparison between the numerical results and the results of the commercial software and the experiments. 
Other than the Hamilton and FE numerical method in building the damping beam model, other researchers proposed many other modeling techniques for the numerical representation of continuous/discontinuous damping beam. Kung and Singn [38] calculated the natural frequencies and loss factor using the Rayleigh-Ritz energy method and modal-strain energy technique for 3-layer patch damping structure. These approximate modeling methods also extended to rectangular damping patch of plates and shells with a viscoelastic core.

Zhang and Sainsbury (2000) [39] combined Gerlerkin orthogonal function with the traditional finite element method, and successfully applied it to the vibration analysis of the damped sandwich plates. The numerical simulation results were described as well as showing its accurate and efficient computational performance.

The research of damped structure is also used in developing models for objects with irregular geometry such as cylinder[40], patch floor [41], etc. Hua et al. [40] proposed an energy-method based on the analytical method as well as on the regular FEM in modeling a fluid-filled cylindrical shell with a viscoelastic core. The confidence of its accuracy and reliability was gained from the comparison of the 
numerical results with experiments.

\subsubsection{Modern Damping Models}

While the FEM takes the dominant position in the simulation area, many researchers looked for proper mathematical model to represent the damping behavior of viscoelastic material, as well as to make the damping model available in commercial FEM software packages [42]. Currently, the FEM commercial software incorporate the traditional damping models such as viscous/hysteretic damping [43], Rayleigh/ Proportional damping and the Prony Series [44], all of which incorporate damping energy dissipation in the time domain. However, none of these damping models are able to capture the damping behavior in the frequency domain, which is the most interesting issue in the research in vibro-acoustical area. The disadvantage of these damping models raised considerable interest among researchers and motivated them to develop a damping model for viscoelastic material with a frequency dependence that is compatible with the FEM technique. There are two categories of mathematical models, most popular in the research of viscoelastic damping: derivative type and integral type. 
The "Fractional Derivative" is essentially the representative damping model in the derivative form. It is still active in the research of recent years. Bradley and Torvik [45] proposed the fractional derivative constitutive relationship of viscoelastic material in 1983. This damping model not only described the material properties of viscoelastic damping, but rather established the close-form equation compatible with the FEM technique. Compared with the other integral-form models, the fractional derivative is only able to capture the relatively weak frequency-dependent information; however, it was an important milestone in the area of damping research.

In recent years, the research interests concentrated on developing the time-domain integral form and frequency-domain damping model derived from integral form that can be applied to current FE models. Being dissatisfied with the fractional derivative operators, the researchers developed the time-domain (ODE) Ordinary Differential Equation combined with the integral-form damping models that is more convenient to solve. Such kinds of proposed damping models are roughly classified into: ADF type (ATF [46] /ADF [47] model), mini-oscillator type (Biot [48], GHM [49, 50]) and the others (Yiu [51], Adhikari [52], etc) 
Lesieutre and Bianchini [46] mathematically modeled the relaxation behavior of viscoelastic material in terms of Augmenting Thermodynamic Field (ATF) in 1989. Initially introducing the single augment field, this damping model provided the ability to represent the light-damping behavior with the application of a 1D viscoelastic structure. In the subsequent research, using a series of augment fields, the ATF model is able to model the damping material of higher loss factor with the weak frequency dependence. Remedying the limitation of 1D application, Lesieutre and Lee [47] proposed an Anelastic Displacement Field (ADF) technique in 1996 and successfully extended its application from the 1D problem to the 3-layer sandwich beam and 3D problems.

As far as the mini-oscillator damping models are concerned, the complex shear modulus (the function with respect to both frequency and temperature), can be expressed in mechanical terms as the series of mini-oscillation perturbators. In fact, both the "mini-oscillator" and ADF type models have the obvious similarities: they increase the number of DOF to gain the frequency independence; in other words, they use the additional dissipative coordinates to accurately model the relaxation function of viscoelastic damping material. The "mini-oscillator" damping models 
such as Biot and GHM are based on the application of Laplace-Domain relaxation function and retransformation from the Laplace domain to the time-domain ODE associated with the conventional FEM. ADF type models, on the other hand, are not transformation based and they directly result in the formulation of time domain

Biot (1955) [48] first proposed the 1st order relaxation function with the introduction of the "dissipative variables" into the dynamic ODE, from his theory of irreversible thermodynamics. In 2006, Zhang et al. [53] utilized the Biot model to describe the dynamic behavior of the viscoelastic structure. The dimension reduction technique and non-linear curve-fitting procedure are also discussed in Zhang's publication.

Golla, Huges and Mactivish [50] developed another mini-oscillator damping model called "GHM" by the usage of 2nd order relaxation function in 1985 and 1993. Compared with the Biot model, the GHM model has more complicated expression and also requires better performance of the computational tool due to the higher dimension of the mathematical equation in which the 2 nd order relaxation function resulted. Unlike the Biot model, GHM model has the additional mass terms in the dissipative variables in the mechanical analogy. 
The popularity of these integral-form damping models in recent years brought two research interests: non-linear curve fitting and dimension reduction. The advanced curve-fitting technique in the damping models guarantees the accuracy of the numerical representation of the actual shear modulus data from the experiment. The dimension reduction technique increases the computational efficiency due to the additional orders of equation used to gain the frequency independence of the frequency-form damping model.

Zhang et al. [54] converted the nonlinear curve-fitting problem in frequency domain with respect to the GHM parameters, into the constrained nonlinear optimization problem. The efficiency and correctness can be seen from the comparison between the experimental complex shear modulus and the curve fitting result of $\mathrm{ZN}-1$ viscoelastic material.

Park, Inman and Lam [55] examined the GHM damping model with the application to the FE method associated with the Guyan reduction technique. The numerical example in this research leads to a FE model applied to the GHM dynamic equation quantitatively without increasing the number of dimension. 


\subsubsection{Control Strategies}

The research activities for the control purpose were launched by Plump and Hubbard Jr. in 1986 [56]. Their work introduces the concept of ACL that could improve the performance of passive constrained-layer treatment. They tried to establish the proportional relationship between energy dissipation and vibration amplitude.

The advantages of their work are:

"(i) their capacity to increase actively the shear strains in the viscoelastic material, through the piezoelectric actuator, thus improving energy dissipation;

(ii) Combination of the performances at higher frequencies of viscoelastic treatments and, at very low frequencies, of the active control through piezoelectric actuation;

(iii) Increase in the robustness of active control, insofar as, in the event of operation fail, the system remains passively damped."

However, Lagnese (1984) [57] showed that the energy dissipation (measured by loss factor) do not decrease with strain amplitude. Later, Austin (1991) et al. [58] stated that the effectiveness of a constrained-layer didn't decrease with vibration amplitude 
using an example.

The research of the Active Constrained Layer (ACL) damping, achieved by using the piezoelectric materials as the actuator, allows the active the control of the structural vibrations to increase shear in the VEM. As far as the constrained-layer damping mechanism is concerned, the localization of the dissipation on the edge of layers makes the optimization of constrained layer treatments more difficult since different boundary conditions and possible connections of the layers are of significance.

From 1990s, many different configurations of ACL were proposed in the literature based on the relative position of viscoelastic and piezoelectric layer as well as the type/position of sensor and actuator.

Using the ACL configuration proposed by Plump and Hubbard Jr. (1986) [56], Liao et al. (1997) [59] and Shen $(1994 ; 1996)[60,61]$ measure the tip deflection of beam using optical sensor, by adding more system damping through the feedback to piezoelectric actuator. Yet, Varadan et al. (1996) [62] bonded a piezoelectric sensor next to Liao and Shen's hybrid treatment . 
Lesieutre and Lee (1996) [36] tried to increase the system robustness by measuring the surface strain of the beam bottom. Unfortunately, the performance is not ideally in the higher frequency range.

Baz (1997) [63] and Trindade, Benjeddou, and Ohayon (2000) [64] utilized segmented ACL introducing the additional hybrid damping to the control system by bonding two symmetric treatments on the beam surfaces.

Agnes and Napolitano (1993) [65], Yellin and Shen (1996) [66] and Huang et al. (1996) [67] investigated the self-sensing actuators as an ACL to simultaneously actuate and sense the structural vibration. This configuration has many advantages leading to a collocated control system developed by Dosch, Inman, and Garcia (1992) [68]

The following studies(Baz et al. (1993; 1995) [69, 70] and Baz (1998) [71]) by sandwiching a piezoelectric sensor between the viscoelastic layer and structure.

Without using the complicated self-sending actuator, this configuration leads to excellent co localization of both actuator and sensor. 
A good literature review on ACL is summarized by Trindade and Benjuddou (2002) [72].

\subsubsection{Damping Identification and Optimization}

\section{A. Identification}

Many papers have been published in the identification of the damping properties for the laminated structure. In most research studies, the damping identification technique is considered as an optimization problem. Generally speaking, the optimization technique has shown good applicability for numerically identifying the material parameters for the damping structure.

Kim and Lee [73] proposed a methodology for the damping identification procedure for a two-layer extensional damping beam and used experiments to verify numerical results. The Fractional Derivation damping model with the Arrhenius and WLF equation was used to decrease the number of unknowns during the identification (optimization) process, while temperature shifting factor was taken into consideration. 


\section{B. Optimization}

The objective of the optimization research on laminated damping structures is to maximize the loss factor, in order to minimize the peak value based on either vibration or acoustical analysis. This is done by determining the optimal parameters such as number of layers, sizing, placement, etc.

Starting from Plunkett and Lee [74] on their theoretical optimal length investigation of surface damping treatments, modern researchers examined the optimal design for both constrained [75-80] and unconstrained [81, 82] layer damping treatments in order to maximize the loss factor and reduce the vibro-acoustical responses.

Kruger and Mann [83] conducted experimental measurement in the noise control problem of a constrained-layer damping patches structure. They also investigated the optimal placement and simulation using boundary-element method.

Many researchers, such as Azzouz and Ro [84], Gibbs and Cabell [85], Zheng and Cai [86] proposed the optimal design of the constrained-layer damping model. They 
base their research on the assumed-modes method of analytical beam model in obtaining the frequency-domain response, and then solve the acoustical problem using Rayleigh's approach. They fail to consider the temperature effect on viscoelastic damping material, and the difficulties of arbitrary geometry or boundary condition. These need to be addressed in future research.

Hao and Rao [87] carried out the optimum design of a three-layer sandwich beam for vibration analysis in 2005. In this research, the numerical model of the damping system is a comprehensive formulation for a three-layer asymmetrical sandwich beam with two different damping materials adjacent to each other. The criterion of the optimization is to minimize the mass of the structure while maximizing the system damping.

In 2007, Lee [88] published the semi-coupled vibro-acoustical analysis and optimization of a simply-supported three-layer sandwich beam. The modal superposition method was utilized to investigate the vibration problem with the fractional derivative as the damping model. The interior acoustical problem was studied by BEM numerical technique. The optimization problem was established to figure out the appropriate sizing parameters of the sandwich beam needed for 
attenuating the peak sound pressure on certain frequencies. The numerical result was provided to verify the effectiveness of this proposed research. 


\subsection{Concepts and Properties of Viscoelastic Material}

Because the complication of the physical structure leads to the non-linear material's characteristics, it is difficult to establish an accurate mathematical representation of the mechanical performance of the viscoelastic damping materials. In general, the constitutive relationship between stress and strain for the damping material can be greatly affected by: time, temperature, frequency, type of deformation, etc. The recent application of the viscoelastic material in vibration and noise reduction task was discussed in Rao's review [11].

\subsubsection{Temperature-Frequency Dependent Properties}

By increasing the temperature or decreasing the frequency, four regions can be observed consecutively for the viscoelastic damping material: Glassy, transition, rubbery and melt (flow) region as shown in Figure 2.4. 


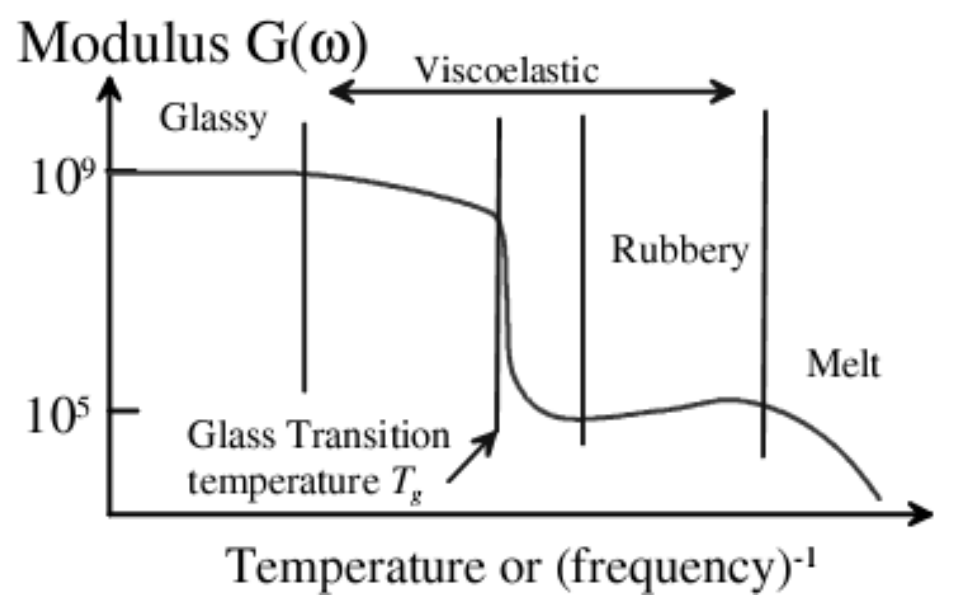

Figure 2.3 Variation of shear modulus of a viscoelastic material with varying

temperature/frequency

The viscoelastic material shows variations in the shear response in the experiment, typically conducted by applying a constant shear stress on the specimen and then measuring the shear strain as a function of time. In this static experiment, creeping and relaxation are two major phenomena, both of which are highly temperature/frequency dependent. The relaxation response is illustrated as follows, by plotting the shear strain as a function of time in different regimes. 


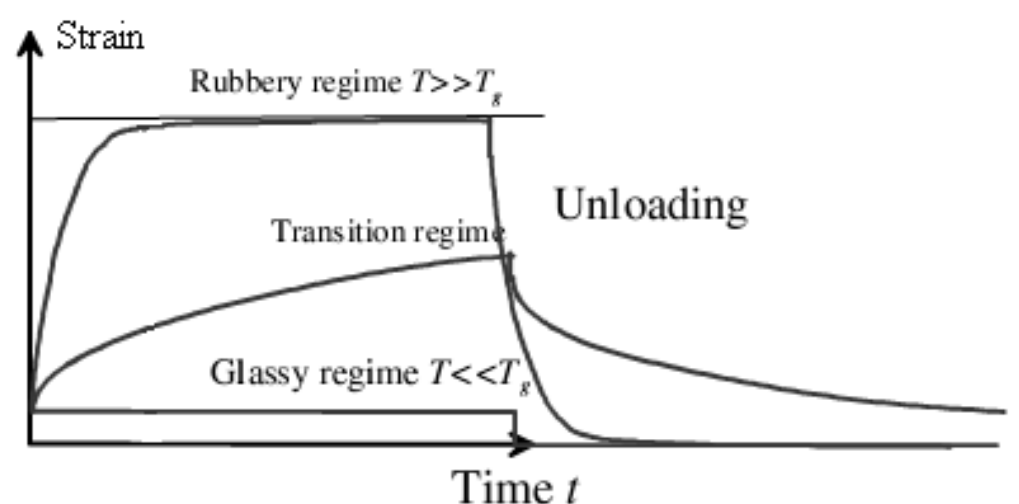

Figure 2.4 Relaxation plot in different regions

Regardless of the region in which the viscoelastic material is, an instantaneous strain always exists with a step change of the stress, and this strain response is almost independent of temperature/frequency. In the glassy region (as plotted by red curve), the viscoelastic material shows essentially the elastic property. In the rubbery region (blue curve), the material performs the relaxation phenomena- gradual change of the stress when the material is holding a constant strain. The strain shows an initial transient response, and then settles to a relatively constant value under the constant stress. The viscoelastic material responses change slowly in the transition region (green curve), compared with the situation in both rubbery and glassy regions. If the constant stress is removed, the strain will eventually return to the zero, although the material in the transition region takes the longest restoring time. 


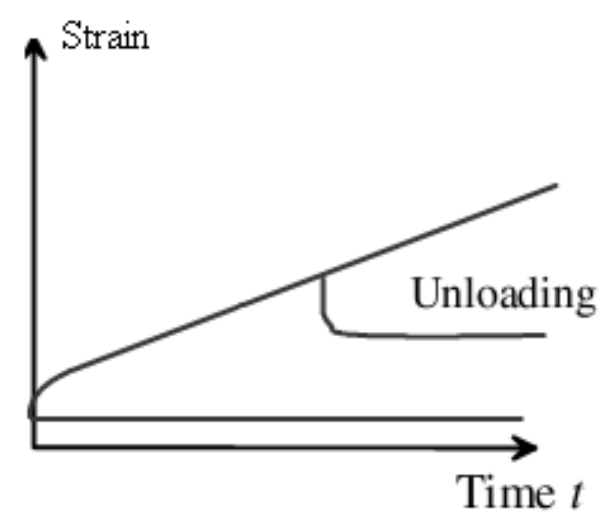

Figure 2.5 Creeping plot in different regions

Similar with the relaxation plot, there is an instantaneous strain initially independent of temperature/frequency, and the material shows elasticity in the glassy region. In the rubbery region, the creeping phenomena is acting. Here, the change of strain is slow and progressive under the constant stress. As shown in Figure2.6, the strain starts from a short non-linear section, then follows the linear relationship with time and this rate of change is strongly temperature/frequency dependent. The deformation of the viscoelastic material is irreversible in the glassy region--i.e.; when the constant stress is removed, the strain will not return to zero in the experiment. 


\subsubsection{Damping Mechanism}

It is popular to use numerical approximations to describe the mechanical characteristic of viscoelastic damping materials. Due to active research in this field over the years, numerous mathematical models for the viscoelastic damping material have been successfully established. These damping models can be categorized as: the derivative type and the integral type; and both types can be expressed as time-domain or frequency-domain form.

\section{A. Derivative Type}

Maxwell and Kelvin damping models, -- the fundamental idealizations on which other damping models are based--, are composed of spring-dashpot systems in series and in parallel respectively. These two simplest time-domain damping models have a very obvious disadvantage: each model only explains either relaxation or creep phenomena. The following table (2.1) explains all aspects of these two damping models in detail: 
Chapter 2.3-Concepts and Properties of Viscelastic Material

Table 2.1 Explanation of Maxwell and Kelvin damping model

\begin{tabular}{ccccc}
\hline \hline Model & Type of link & Explains & $\begin{array}{c}\text { Constitutive } \\
\text { Relationship }\end{array}$ & \\
\hline Maxwell & crosslink & relaxation & $G \sigma+\eta \frac{d \sigma}{d t}=G \eta \frac{d \varepsilon}{d t}$ & \\
Kelvin & Uncross & steady-stat & & \\
& e creep & $\sigma=G \varepsilon+\eta \frac{d \varepsilon}{d t}$ & \\
parallel & & & \\
\hline \hline
\end{tabular}

Unfortunately, Maxwell/Kelvin models do not contain enough information to give an accurate quantitative fit to any polymer over an extended period of time. However, the generalized standard model conquered this limitation and the expression is as follows:

$$
\sigma+\sum_{n=1}^{\infty} \alpha_{n} \frac{d^{n} \sigma}{d t^{n}}=E \mathcal{E}+\sum_{n=1}^{\infty} \delta_{n} \frac{d^{n} \mathcal{E}}{d t^{n}}
$$

where

$\mathrm{E} \quad=$ Young's modulus

$a_{\mathrm{n}}=$ stress dissipation coefficient

$\hat{\mathrm{a}}_{\mathrm{n}}=$ strain dissipation coefficient

$\mathrm{n}=$ order of derivative 
Obviously, this damping model provides enough coefficients to curve-fit the experimental data. However, due to its computational complication, very few recent papers have applied this damping model. In addition, the research in other types of derivative-form damping models such as fractional derivative is also very active; but these derivative-form models are often incompatible with conventional FEM techniques. In other words, these derivative-form damping models have not made any significant contributions that would be of use in industrial applications.

B. Integral Type

For practical purposes, the constitutive relationship of the viscoelastic materials is usually modeled as the integral form, instead of the derivative form. Based on the Boltzmann Superposition Principle [89], the integral expression of damping model is of the form:

$$
\sigma(t)=G_{r} \varepsilon_{0}+\int_{0}^{t} G(t-\tau) \frac{\partial \varepsilon(\tau)}{\partial r} d r
$$

Or

$$
\varepsilon(t)=\frac{\sigma_{0}}{G_{u}}+\int_{0}^{t} J(t-\tau) \frac{\partial \sigma(\tau)}{\partial r} d r
$$


in which

$\mathrm{J}=$ creep compliance

$\mathrm{G}=$ relaxation function

$\mathrm{G}_{\mathrm{r}}=$ relaxed shear modulus

$\mathrm{G}_{\mathrm{u}}=$ unrelaxed shear modulus

The mathematical relationship between $\mathrm{J}$ and $\mathrm{G}$ in the Laplace domain is:

$$
J(s) \bullet G(s)=\frac{1}{s^{2}}
$$

Specifically, the ODE solution of the Maxwell damping model represents the relaxation response of a step increment in strain:

$$
\sigma=\left(G_{1}+G_{2} e^{-t / t_{1}}\right) \varepsilon_{0}
$$

where $t_{1}$ is the time constant and equal to $G_{2} / \eta$

The integration of this equation stands for summing up this relaxation response over a certain time period:

$$
\sigma=\int_{0}^{t}\left[G_{1}+G_{2} e^{-\frac{(t-s)}{t_{1}}}\right] \dot{\varepsilon}(s) d s
$$

Unfortunately, this model still provides very few of the parameters that can give a 
satisfactory curve-fit to the experiment. However, an improved model can be made by connecting numerous Maxwell elements in series, then adding a spring in parallel with the whole system as shown in the figure below.

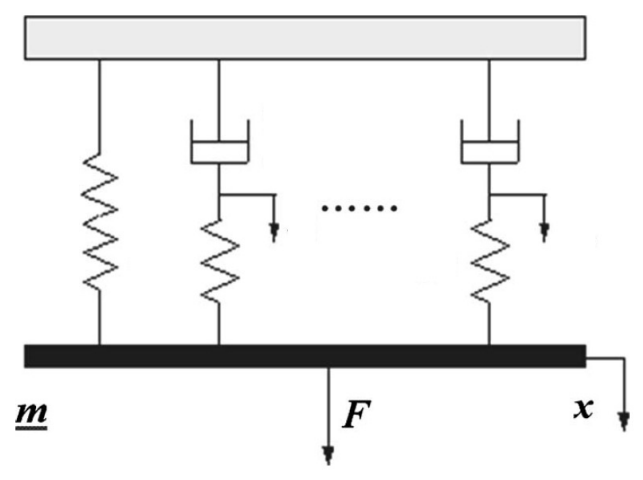

Figure 2.6 Mechanical analogue on series of Maxwell elements

The constitutive representation of the step response for the increment in strain is as follows:

$$
\sigma=\left[G_{\infty}+\sum_{i=1}^{N} G_{i} e^{-t / t_{i}}\right] \varepsilon_{0}
$$

where $G^{\infty}$ is the long-term (elastic) response, i.e.; the steady-state stiffness (the parallel spring); and $G_{i}$ and $t_{i}$ when $i=1 \ldots N$ stand for the stiffness of each Maxwell element and the time constant respectively. 
By doing so, more complicated relaxation behavior can be explained by the following constitutive equation:

$$
G(t)=G_{\infty}+\sum_{i=1}^{N} G_{i} e^{-t / t_{i}}
$$

With the sum of exponentials known as "Prony series", the following relaxation modulus function can be computed through the stress response to an arbitrary strain history from the experiment.

C. Dynamic Performance and frequency-domain damping models

The coefficients in the time-domain damping models from previous chapters, both derivative and integral form, are determined by relaxation or creep static test.

Generally speaking, the data provided by static test is reliable roughly from $10 \mathrm{sec}$ up to 10 years. However, many engineering viscoelastic materials have very short response time under the external load. In this case, the dynamic mechanical performance between 0 and $10 \mathrm{sec}$ is of vital importance for industrial usage.

Additionally, the dynamic performance for the viscoelastic damping materials is greatly affected by many factors such as: temperature, frequency, excitation type, etc. 
Therefore, an engineering method to accurately represent the shear modulus influenced by external factors is needed.

The complex shear modulus is usually used to describe the phase difference of the stress-strain relationship due to the excitation with the following form:

$$
G(\omega)=G^{\prime}+j G^{\prime \prime}(\omega)=G^{\prime}\left(1+j \eta_{s}\right)
$$

where $\eta$ is the loss factor and physically stands for the energy dissipated per radian divided by the total energy. The value of complex shear modulus depends on the different exciting frequencies and ambient temperature.

In the last section, the Prony series representing a series of Maxwell damping models connected in parallel is given. We can rewrite the expression using Biot parameters:

$$
G(t)=G^{\infty}+\sum_{i=1}^{N} G^{\infty} a_{i} e^{-\frac{t}{b_{i}}}
$$

Taking the Laplace Transform and multiplying the Laplace variable "s" on both sides, the equation becomes:

$$
s \widetilde{G}(s)=G^{\infty}\left[1+\sum_{k=1}^{m} a_{k} \frac{s}{s+b_{k}}\right]
$$


The equation above is the expression of Biot damping model. With $s=\sigma+j \omega$, considering the case when $\sigma$ is equal to zero, the Equation 2.10 yields:

$$
s \widetilde{G}(j \omega)=G^{\infty}\left[1+\sum_{i=1}^{N} \frac{a_{i} \varpi^{2}}{b_{i}^{2}+\varpi^{2}}\right]+j G^{\infty}\left[1+\sum_{i=1}^{N} \frac{a_{i} b_{i} \varpi}{b_{i}^{2}+\varpi^{2}}\right]
$$

where $\mathrm{N}$ stands for number of series terms; $G^{\infty}$ is the long-term shear moduli, and $\mathrm{a}_{\mathrm{i}}, \mathrm{b}_{\mathrm{i}}$ are the Biot constants. By determining the number of terms in Equation $2.11, G^{\infty}, \mathrm{a}_{\mathrm{i}}$ and $\mathrm{b}_{\mathrm{i}}$ can be curve-fitted in the frequency domain from the experimental data or the data sheet provided by the manufacturer.

Hence the Prony series can be converted to the form of complex shear modulus by means of the Laplace transformation, and Equation 2.11 is a function of the radiant frequency. The Biot shear modulus function essentially approximates the experimental data in the width of a frequency range; yet the complex shear modulus is the single data spot in a certain frequency.

Besides the Biot damping model, many researchers have proposed several frequency-domain mathematical models to represent the frequency dependence of the viscoelastic material's properties which are compatible with the modern numerical engineering techniques such as FEM. The following Table 2.2 lists the 
various mathematical damping functions and the researchers in chronological order..

Table 2.2 Proposed frequency-domain damping models

\begin{tabular}{cc}
\hline \hline Damping Function in the Freq Domain & Buthor, Year \\
\hline$s(s)=G^{\infty}\left[1+\sum_{k=1}^{n} \frac{a_{k} s}{s+b_{k}}\right]$ & Biot,1955 \\
$G(s)=\frac{E_{1} s^{\alpha}-E_{0} b s^{\beta}}{1+b s^{\beta}}$ & Bagley and Torvik,1983 \\
$0<\alpha<1 ; 0<\beta<1$ & Leisieutre,1992 \\
$G(s)=\sum_{k=1}^{n} \frac{\Delta_{i} s}{s+\Omega_{i}}$ & McTavish and Hughes, 1993 \\
$s G(s)=G^{\infty}\left[1+\sum_{k=1}^{n} a_{k} \frac{s^{2}+2 \xi_{k} \omega_{k} s}{s^{2}+2 \xi_{k} \omega_{k} s+\omega_{k}^{2}}\right]$ & \\
$G(s)=\tau_{0} s+\sum_{k=1}^{n} \frac{\alpha_{i} \tau_{i} s}{\tau_{i} s+1}$ & Yiu,1993 \\
$G(s)=c \frac{1-e^{-s t_{0}}}{s t_{0}}$ & \\
\hline \hline
\end{tabular}

In sum, the figure below shows the mathematical relationship of the relevant damping models in this research. The green color symbolizes the time-domain data, and blue color stands for the data in the frequency domain. 
Chapter 2.3-Concepts and Properties of Viscelastic Material

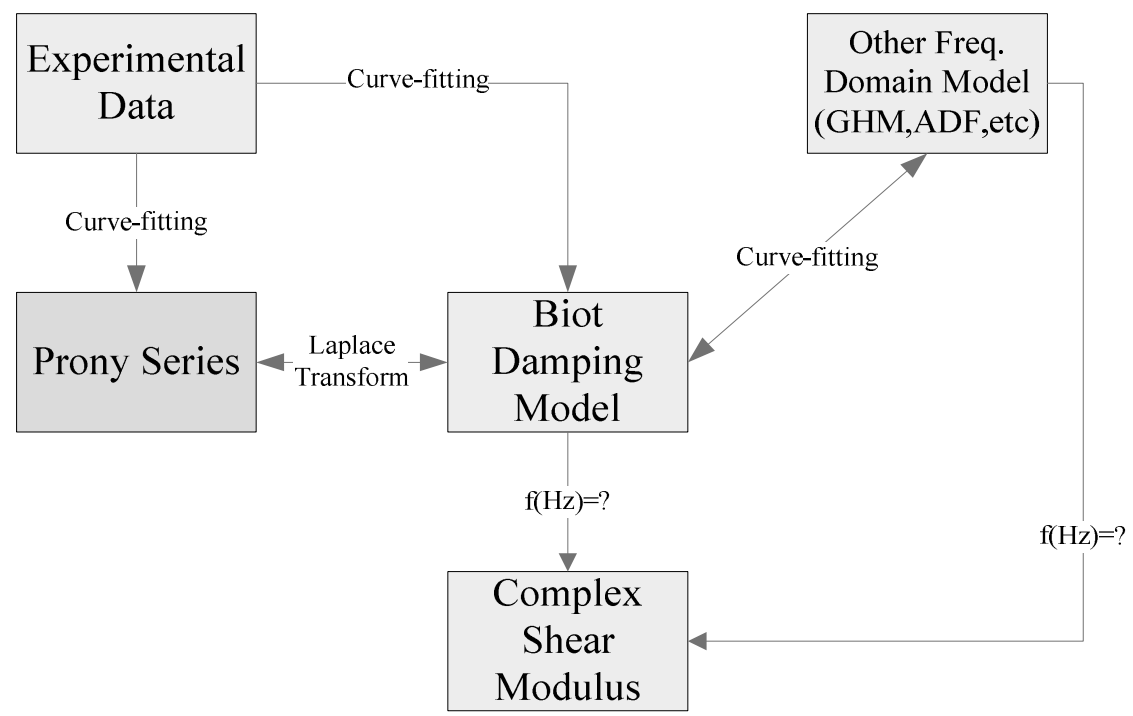

Figure 2.7 Relationships of different damping models 


\section{Research Overview}

The semi-coupled vibro-acoustical problem is proposed in this research. The technical term "semi-coupled" implies that the vibration produces the acoustical responses, yet the sound doesn't induce the vibration of the structure. To be more detailed, the multiple-layer sandwich beam vibrates due to the external excitation on the structure, and the change of surface velocity due to the vibration produces the change of the acoustical waves scattered around subsequently. However, the sound pressure produced by the vibration cannot affect the vibration responses of the sandwich beam. The visualization of this relationship is plotted in the following figure.

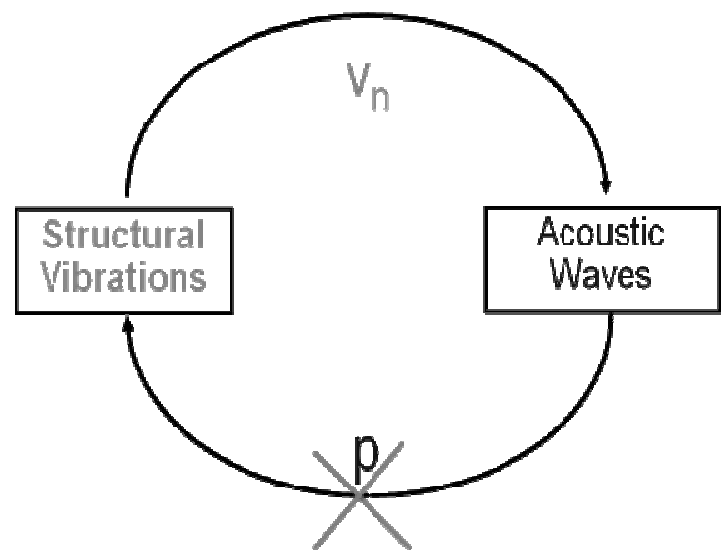

Figure 3.1 Schematic diagram of the semi-coupled problem 
The flow chart below visualizes the key steps as well as the data flow that will be dealt with in this research. This research can be classified into two parts: vibration and acoustical analysis. In the vibration analysis, the multiple-layer sandwich beam is discretized by the FE modeling technique and analyzed by the dynamic equation using the Biot damping model. The curve-fitting technique is needed to convert the experimental data into Biot constants that can be plugged into the dynamic equation. The dimension reduction technique removes zero items in the eigenvalue problem when the dynamic equation is established, and increases the computational efficiency at the same time. Eventually, the Frequency Response Function (FRF) in the frequency domain is the output of the vibration analysis. The node velocities of the FE numerical model can be determined through the FRF with the arbitrary excitation. With respect to the acoustical analysis, the BE-based 2D interior problem is studied in this research. The distribution of sound pressure can be computed through the result of node velocity obtained from the vibration analysis. The detail of the acoustical analysis will be discussed in this research. 


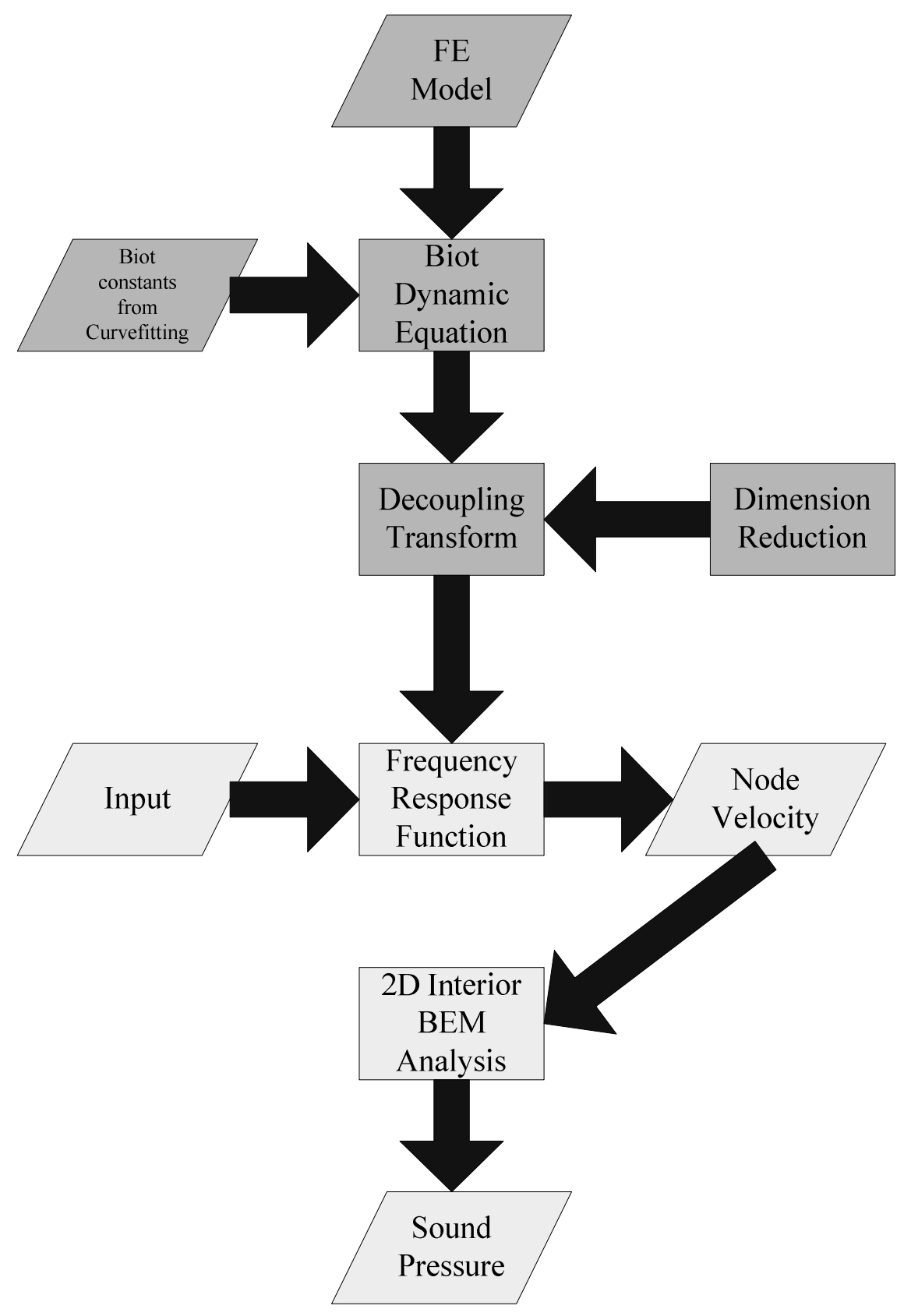

Figure 3.2 Flow chart of the key steps in this research 


\section{Vibration Analysis}

\subsection{FE Modeling of the Multiple-Layer Beam}

The modeling of the multiple-layer sandwich beam will be discussed in this section. The elastic beam and the constrained damping layer are two fundamental components in this modeling task. The concept of transfer matrix is used to convert the local coordinates to the global coordinates. The two components are then assembled and used to construct the complete model of the structure with arbitrary number of layers. The approach is demonstrated for modeling beam structures. The technique can be applied to structures with multiple-layered plate or shells in the same manner as long as the system mass and stiffness matrices are made available.

The following demonstrates the details for modeling the beam system.

\subsubsection{Two Fundamental Components in a Multiple-Layer Beam}

A. Modeling of the Elastic Layer

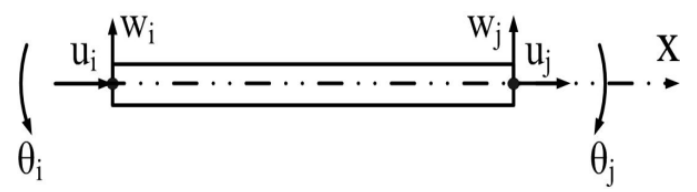

Figure 4.1 Configuration of the elastic layer showing the DOF 
The FE model shown in Figure 4.1 contains 2 nodes, 6 DOF. The displacements of each node can be expressed as:

$$
\{\delta\}_{\text {elastic }}^{e}=\underbrace{\left(\begin{array}{lll:lll}
w_{i} & \theta_{i} & u_{i} & w_{j} & \theta_{j} & u_{j}
\end{array}\right)^{T}}_{6 \times 1}
$$

Therefore, the displacement of any point inside the element would be:

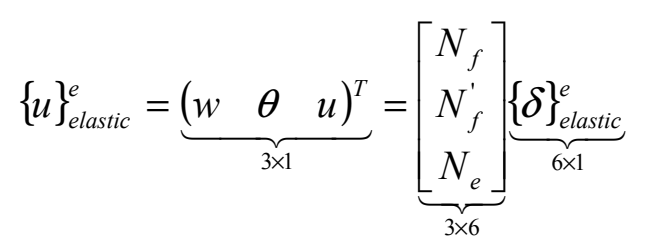

where the shape functions are as follows:

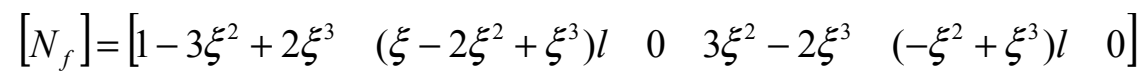

$$
\begin{aligned}
& {\left[N_{f}^{\prime}\right]=\left[\frac{\partial N_{f}}{\partial x}\right]=\frac{1}{l}\left[\frac{\partial N_{f}}{\partial \xi}\right]} \\
& {\left[N_{e}\right]=\left[\begin{array}{llllll}
0 & 0 & 1-\xi & 0 & 0 & \xi
\end{array}\right]}
\end{aligned}
$$

$\xi=x / l, \xi \in[0,1]$, and $\xi_{\text {stands for the local coordinate; letter } 1 \text { is the length of }}$ beam along $\mathrm{x}$ as the horizontal axis.

Next, the stiffness matrix can be derived based on the energy method:

$$
\left[K_{e}\right]_{\text {elastic }}^{e}=\int_{l}\left[E A \varepsilon^{2}+E I \varepsilon_{\theta}^{2}\right] d x
$$

where the first and second term in the integration are the extensional strain energy and the bending strain energy of the elastic layer respectively. 
Applying the shape function (Equation 4.3) yields the element stiffness matrix in the local coordinate form:

$$
\left[K_{e}\right]_{\text {elastic }}^{e}=\int_{0}^{1} \frac{E A}{l} \underbrace{\left[\frac{\partial N_{e}}{\partial \xi}\right]^{T}}_{6 \times 1} \underbrace{\left[\frac{\partial N_{e}}{\partial \xi}\right]}_{1 \times 6} d \xi+\underbrace{\int_{0}^{1} \frac{E I}{l}\left[\frac{\partial^{2} N_{f}}{\partial^{2} \xi}\right]^{T}\left[\frac{\partial^{2} N_{f}}{\partial \xi^{2}}\right] d \xi}_{6 \times 6}
$$

where,

A: Cross-sectional area of the elastic layer,

E: Young's Modulus of the elastic layer,

1: Longitudinal length of elastic layer, and

I: Moment of Inertia of elastic layer.

Similarly, the element mass matrix can be expressed as:

$$
\underbrace{[M]_{\text {elastic }}^{e}}_{6 \times 6}=\int_{0}^{1} m l(\underbrace{\left[N_{f}\right]^{T}}_{6 \times 1} \underbrace{\left[N_{f}\right]}_{1 \times 6}+\left[N_{e}\right]^{T}\left[N_{e}\right]) d \xi
$$

where $\mathrm{m}$ is the mass of the unit length in this elastic layer.

B. Modeling of the Constrained damping layer

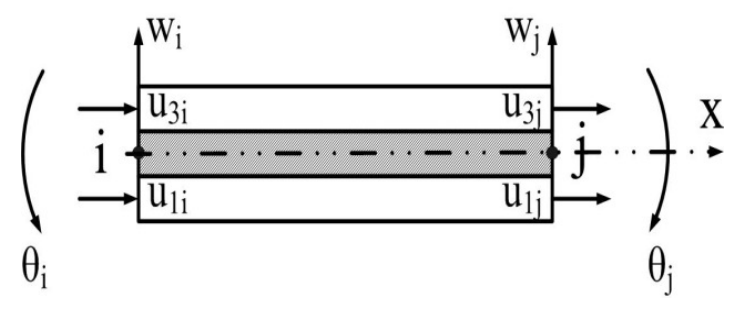

Figure 4.2 Configuration of the constrained damping layer showing the DOF 
The construction of the FE model of the constrained damping beam consisting of a damping layer sandwiched between two outer layers is shown in Figure 4.2 The major assumption of this three-layer structure is that these three layers share the identical transverse and rotational displacement; however, all three layers have different axial displacement allowing the shear deformation of the constrained damping layer. Figure 4.2 illustrates an FE model consisting of 2 nodes, and 8 DOF in each element. The nodal displacement vector is:

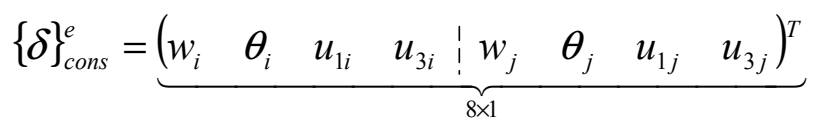

In Figure 4.2, the top and bottom layers can be treated as the elastic layers, as discussed earlier in this section. Due to the subordinate relationship of the nodal displacement between the three-layered 8 DOF sandwich element and the elastic layer element with $6 \mathrm{DOF}$, the element stiffness and mass matrix can be derived through a transportation matrix.

By comparing the nodal displacements of the elastic layer (Equation (4.1)) and constrained layer system (Equation (4.7)), the mathematical relationship between the two vectors can be established as: 


$$
\underbrace{\{\delta\}_{\text {elastic }}^{e}}_{6 \times 1}=[\underbrace{\left[T_{1}\right]}_{6 \times 8} \underbrace{\{\delta\}_{\text {cons }}^{e}}_{8 \times 1}
$$

in which the transfer matrix can be written as: $\left[T_{1}\right]=\left[\begin{array}{llllll}e_{1} & e_{2} & e_{3} & e_{5} & e_{6} & e_{7}\end{array}\right]^{T}$ and each $\mathrm{e}_{\mathrm{i}}$ means the following vector:

$$
e_{i}=\left(\begin{array}{lllllll}
0 & 0 & \ldots & \ldots & \underbrace{1}_{i^{\text {th }}} \begin{array}{clll}
1 & \ldots & \ldots & 0
\end{array})^{T}
\end{array}\right.
$$

The dimension of the transfer matrix is purely determined by the DOF of the source as well as the target nodal vector. Therefore, the element stiffness and mass matrices of the top layer can be calculated by: $\left[T_{1}\right]^{T}\left[K_{e}\right]_{\text {elastic }}^{e}\left[T_{1}\right]$ and $\left[T_{1}\right]^{T}[M]_{\text {elastic }}^{e}\left[T_{1}\right]$ The same procedure can be applied to the bottom layer by means of the transfer matrix as:

$$
\left[T_{3}\right]=\left(\begin{array}{llllll}
e_{1} & e_{2} & e_{4} & e_{5} & e_{6} & e_{8}
\end{array}\right)^{T} ; e_{i}=\left(\begin{array}{llllllll}
0 & 0 & \ldots & \ldots & \underbrace{1}_{i^{\text {h }} \quad \text { place }^{1}} & \ldots & \ldots & 0
\end{array}\right)^{T}
$$

Neglecting the axial and bending strain energy of the viscoelastic damping layer in this three-layer structure, only the shear strain energy is taken into consideration when the element stiffness matrix of the damping layer is derived. Figure 4.3 visualizes the displacement relationship in this three-layer structure. 


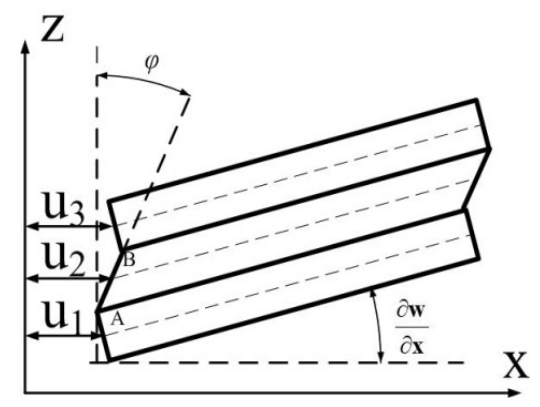

Figure 4.3 Deformation relationship of the viscoelastic core

Here $\mathrm{u}_{\mathrm{A}}$ and $\mathrm{u}_{\mathrm{B}}$ symbolize the horizontal displacement for the corner point of both elastic layers adjacent to the damping layer. In this 3-layer element, $\mathrm{u}_{1}, \mathrm{u}_{2}$ and $\mathrm{u}_{3}$ stand for the horizontal displacement for the base layer, damping layer and the constrained layer, $\mathrm{h}_{1}, \mathrm{~h}_{2}$ and $\mathrm{h}_{3}$ are the thickness of the three different layers respectively.

$$
u_{B}=u_{3}+\frac{\partial w}{\partial x} \frac{h_{3}}{2} ; u_{A}=u_{1}+\frac{\partial w}{\partial x} \frac{h_{1}}{2}
$$

The rotational angle along the $\mathrm{y}$ axis for the damping layer due to the axial mismatch of the two elastic layers is:

$$
\varphi=\frac{u_{B}-u_{A}}{h_{2}}
$$

Therefore, the total shear strain would be the accumulation of the $\varphi$ and the angle which the consistent rotational angle contributes to the damping layer. Thus shear strain of the damping layer can be expressed as: 


$$
\gamma_{2}=\frac{u_{3}-u_{1}}{h_{2}}+\frac{h_{0}}{h_{2}} \frac{\partial \omega}{\partial x} ; h_{0}=\frac{1}{2}\left(h_{1}+h_{3}\right)+h_{2}
$$

By applying Equation (4.11-13), Equation (4.13) yields the shape function:

$$
\begin{aligned}
& \gamma_{2}=\underbrace{\left[\frac{N_{e 3}-N_{e 1}}{h_{2}}+\frac{h_{0}}{h_{2}} \cdot \frac{1}{l} \cdot \frac{\partial N_{f 1}}{\partial \xi}\right]^{T}}_{1 \times 8} \cdot \underbrace{\{\delta\}_{\text {cons }}^{e}}_{8 \times 1} \text {,where } \\
& \underbrace{\left[N_{e 1}\right]}_{1 \times 8}=\underbrace{\left[N_{e}\right.}_{1 \times 6}]\left[T_{6 \times 8}\right] ; \quad\left[N_{e 3}\right]=\left[N_{e}\right\rfloor\left[T_{3}\right] \quad\left\lfloor N_{f 1}\right\rfloor=\left\lfloor N_{f} \llbracket \llbracket T_{1}\right] \text {. }
\end{aligned}
$$

Accordingly, based on the energy method, the element viscoelastic stiffness matrix can be derived as:

$$
\left[K_{v}\right]_{c o n s}^{e}=\int_{l} \frac{1}{k} G A \gamma^{2} d x
$$

Where

$\mathrm{A}_{2}$ : Cross-sectional area of the damping layer

$\mathrm{G}_{2}$ : Long term shear modulus of the damping layer

$\mathrm{k}$ : Correction factor of the shear strain energy, for the rectangular cross section, $\mathrm{k}=1.2$

Commonly, only the longitudinal kinetic energy needs to be considered; and the element mass matrix in this layer is:

$$
[M]_{\text {cons }, 2}^{e}=\int_{0}^{1} m_{2} l\left[N_{f 1}\right]^{T}\left[N_{f 1}\right] d \xi
$$

In sum, the element elastic stiffness, the element viscoelastic stiffness and the element mass matrix for this 3-layer component respectively are: 


$$
\begin{gathered}
{\left[K_{e}\right]_{\text {cons }}^{e}=\left[T_{1}\right]^{T} \cdot\left[K_{e}\right]_{\text {elastic }} \cdot\left[T_{1}\right]+\left[T_{3}\right]^{T} \cdot\left[K_{e}\right]_{\text {elastic }} \cdot\left[T_{3}\right]} \\
{\left[K_{v}\right]_{\text {cons }}^{e}=\int_{0}^{1} \frac{G_{2} A_{2} l}{k h_{2} l}\left[\frac{N_{e 3}-N_{e 1}}{h_{2}}+\frac{h_{0}}{h_{2}} \cdot \frac{1}{l} \cdot \frac{\partial N_{f 1}}{\partial \xi}\right]^{T}\left[\frac{N_{e 3}-N_{e 1}}{h_{2}}+\frac{h_{0}}{h_{2}} \cdot \frac{1}{l} \cdot \frac{\partial N_{f 1}}{\partial \xi}\right] d \xi} \\
{[M]_{\text {cons }}^{e}=\left[T_{1}\right]^{T} \cdot[M]_{\text {elastic }} \cdot\left[T_{1}\right]+[M]_{\text {cons }, 2}^{e}+\left[T_{3}\right]^{T} \cdot[M]_{\text {elastic }} \cdot\left[T_{3}\right]}
\end{gathered}
$$

\subsubsection{FE Modeling of a Seven-Layer Constrained Damping Beam}

Any damping structure with an arbitrary number of layers can be constructed by the combination of the two fundamental components described above. In terms of mathematical transformation through the transfer matrix, the stiffness and mass matrices of the multiple layers damping beam can be calculated by the element stiffness and mass matrix of the elastic layer as well as the constrained damping layers as discussed in the previous section. This section demonstrates the procedure for building a seven-layer sandwich beam. 


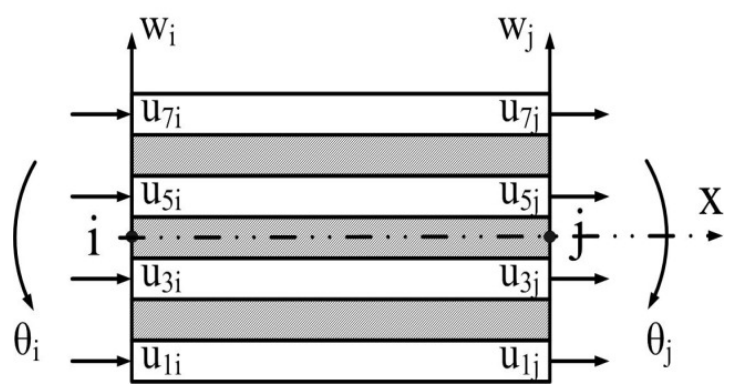

Figure 4.4 Configuration of a seven-layer damping structure showing the DOF

The seven-layer sandwich beam is composed of seven alternating layers, including four elastic layers and three damping layers. The Figure 4.4 above shows the construction of the FE model of a seven-layer sandwich beam, consistent with the assumption in the previous derivation. This FE model contains 2 nodes, 10 DOF, and the node displacement vector is:

$$
\{\boldsymbol{\delta}_{7 \text { layer }}^{e}=\underbrace{\left(\begin{array}{llllll:llllll}
w_{i} & \theta_{i} & u_{1 i} & u_{3 i} & u_{5 i} & u_{7 i} & w_{j} & \theta_{j} & u_{1 j} & u_{3 j} & u_{5 j} & u_{7 j}
\end{array}\right)^{T}}_{12 \times 1}
$$

According to the previous discussion of Equation (4.8), the transfer matrix is as follows to obtain the element stiffness and the mass matrix when the $1 \mathrm{st}, 3 \mathrm{rd}$, 5th and 7 th layers are elastic layers

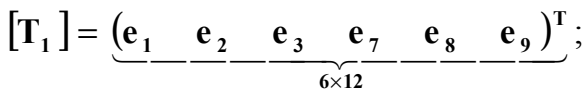

$$
\begin{aligned}
& \left[\mathrm{T}_{3}\right]=\underbrace{\left(\mathbf{e}_{1}\right.}-\underline{\mathbf{e}_{2}}-\underbrace{\mathbf{e}_{4} \quad \mathbf{e}_{7}}_{6 \times 12} \quad \underline{\mathbf{e}_{8}} \quad \underline{\mathbf{e}_{10}})^{\mathrm{T}} ; \\
& \left[\mathrm{T}_{5}\right]=\underbrace{\left(\mathbf{e}_{1}\right.}-\underline{\mathbf{e}_{2}}-\underline{\mathbf{e}_{5} \quad \mathbf{e}_{7}}-\underline{\mathbf{e}_{8}} \quad \underline{\mathbf{e}_{11}})^{\mathrm{T}} ;
\end{aligned}
$$




$$
\left[\mathbf{T}_{7}\right]=\underbrace{\left(\mathbf{e}_{1}\right.}-\underline{\mathbf{e}_{2}}-\underline{\mathbf{e}_{6} \quad \mathbf{e}_{7}} \underline{\mathbf{e}_{8}}-\underline{\mathbf{e}_{12}})^{\mathrm{T}} ;
$$

Similarly, the element stiffness and the mass matrix for the 2 nd, 4 th and 6 th layers as the constrained damping layers can be derived through the transfer matrix as follows:

$$
\begin{aligned}
& \left[\mathbf{T}_{2}\right]=\underbrace{\left(\mathbf{e}_{1}\right.}-\underline{\mathbf{e}_{2}}-\underline{\mathbf{e}_{3}}-\underline{\mathbf{e}_{4} \quad \mathbf{e}_{7}} \mathbf{e}_{8 \times 12}^{\mathbf{e}_{8}} \quad \underline{\mathbf{e}_{9}}-\underline{\mathbf{e}_{10}})^{\mathrm{T}} ; \\
& {\left[\mathbf{T}_{4}\right]=\underbrace{\left(\begin{array}{llllllll}
\mathbf{e}_{1} & \mathbf{e}_{2} & \mathbf{e}_{4} & \mathbf{e}_{5} & \mathbf{e}_{7} & \mathbf{e}_{8} & \mathbf{e}_{10} & \mathbf{e}_{11}
\end{array}\right)^{\mathrm{T}} ;}_{8 \times 12}}
\end{aligned}
$$

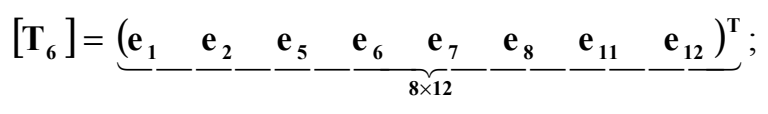

where the notation $e_{i}$ means

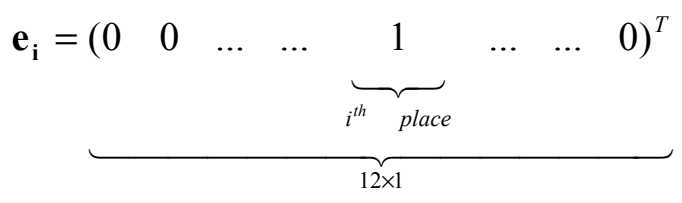

In sum, based on the former equations, and with the parameters of each layer, the element mass/stiffness/damping matrix of the seven-layer sandwich damping beam can be expressed as:

$$
\begin{gathered}
{\left[\mathbf{K}_{\mathbf{e}}\right]^{e}=\mathbf{T}_{1}^{\mathbf{T}}\left[\mathbf{K}_{\mathbf{e} 1}\right] \mathbf{T}_{1}+\mathbf{T}_{3}^{\mathbf{T}}\left[\mathbf{K}_{\mathbf{e} 3}\right] \mathbf{T}_{3}+\mathbf{T}_{5}^{\mathbf{T}}\left[\mathbf{K}_{\mathbf{e} 5}\right] \mathbf{T}_{\mathbf{5}}+\mathbf{T}_{7}^{\mathbf{T}}\left[\mathbf{K}_{\mathbf{e} 7}\right] \mathbf{T}_{7}} \\
{\left[\mathbf{K}_{v}\right]^{e}=\mathbf{T}_{2}^{\mathbf{T}}\left[\mathbf{K}_{v 2}\right] \mathbf{T}_{2}+\mathbf{T}_{4}^{\mathbf{T}}\left[\mathbf{K}_{v 4}\right] \mathbf{T}_{4}+\mathbf{T}_{6}^{\mathbf{T}}\left[\mathbf{K}_{v 6}\right] \mathbf{T}_{6}} \\
{\left[\mathbf{M}_{\mathbf{e}}\right]^{e}=\sum_{i=1}^{7} \mathbf{T}_{\mathbf{i}}^{\mathbf{T}}\left[\mathbf{M}_{\mathbf{e i}}\right] \mathbf{T}_{\mathbf{i}}}
\end{gathered}
$$


Assembling the element matrices and applying the boundary condition by the conventional FE technique [90], the global mass/ stiffness/ damping matrix can be obtained. The viscoelastic damping properties need to be taken into consideration when constructing the global matrices. The dynamic equation and its solution will be discussed in the next section. 


\subsection{Derivation of the Biot Dynamic Equation}

The purpose of this section is to incorporate the damping properties into the discretized system using the FE technique discussed in Chapter 4.1. Unlike the frequency-domain damping model in previous research, this section demonstrates the time-domain Biot damping model with its ability to employ different viscoelastic materials in a damping structure.

The dynamic equation discretized by a numerical implementation such as FEM can be expressed in the following $2^{\text {nd }}$ order ODE form with $\mathrm{M}, \mathrm{C}$ and $\mathrm{K}$ representing the global mass, damping and stiffness matrices respectively:

$$
\mathbf{M} \ddot{\mathbf{x}}+\mathbf{C} \dot{\mathbf{x}}+\mathbf{K x}=\mathbf{f}(\mathbf{t})
$$

By applying the constitutive relationship for two different damping materials with the hereditary integral form to the equation above, the discretized dynamic equation becomes:

$$
\mathbf{M} \ddot{\mathbf{x}}+\mathbf{K}_{\mathrm{e}} \mathbf{x}+\mathbf{K}_{\mathbf{v} 1}\left(G_{1}(t) \mathbf{x}(0)+\int_{0}^{t} G_{1}(t-\tau) \dot{\mathbf{x}} d \tau\right)+\mathbf{K}_{\mathbf{v} 2}\left(G_{2}(t) \mathbf{x}(0)+\int_{0}^{t} G_{2}(t-\tau) \dot{\mathbf{x}} d \tau\right)=\mathbf{f}(t)
$$


In other words, this dynamic system contains two types of viscoelastic damping material, where $\mathrm{K}_{\mathrm{e}}$ stands for the elastic stiffness matrix due to the axial and bending strain energy, and $\mathrm{K}_{\mathrm{v} 1}$ and $\mathrm{K}_{\mathrm{v} 2}$ symbolize the damping matrix of two different types of damping material.

Then the dynamic equation will take the following form by using the Laplace Transform:

$$
\left(s^{2} \mathbf{M}+\mathbf{K}_{\mathbf{e}}+s \widetilde{G}_{1}(s) \mathbf{K}_{\mathbf{v} 1}+s \widetilde{G}_{2}(s) \mathbf{K}_{\mathbf{v} 2}\right) \mathbf{X}(s)=\mathbf{F}(s)
$$

Where s stands for the Laplace variable $(s=\sigma+j \omega)$, and the $\mathrm{sG}_{\mathrm{i}}(\mathrm{s})$ is the core function of the complex shear modulus for the $\mathrm{i}^{\text {th }}$ type of viscoelastic material. In this paper, the Biot viscoelastic damping model will be used, and its complex shear modulus with the series of mini-oscillator perturbing term [7] can be expressed as:

$$
s \widetilde{G}(s)=G^{\infty}\left[1+\sum_{k=1}^{m} a_{k} \frac{s}{s+b_{k}}\right]
$$

in which $G^{\infty}$ is the long-term shear moduli; $\mathrm{a}_{\mathrm{k}}$ and $\mathrm{b}_{\mathrm{k}}$ are the Biot constants. These parameters are positive and can be determined by the non-linear curve fitting from experimental data. For the purpose of simplification, the number of perturbing terms is assumed to be one, and the complex modulus functions of two different viscoelastic materials are: 


$$
s \widetilde{G}_{1}(s)=G_{1}^{\infty}\left[1+a_{1} \frac{s}{s+b_{1}}\right] ; \quad s \widetilde{G}_{2}(s)=G_{2}^{\infty}\left[1+a_{2} \frac{s}{s+b_{2}}\right]
$$

Applying Equation 4.25 to Equation 4.27 and rearranging the terms yields:

$$
\left[s^{2} \mathbf{M}+\mathbf{K e}+\left(G_{1}^{\infty}\left(1+a_{1}\right)-G_{1}^{\infty} \frac{a_{1} b_{1}}{s+b_{1}}\right) \mathbf{K}_{\mathbf{v} 1}+\left(G_{2}^{\infty}\left(1+a_{2}\right)-G_{2}^{\infty} \frac{a_{2} b_{2}}{s+b_{2}}\right) \mathbf{K}_{\mathrm{v} 2}\right] \mathbf{X}(s)=\mathbf{F}(s)
$$

The following procedure will introduce the dissipation coordinates into the dynamic equation in order to eliminate the Laplace variable " $\mathrm{s}$ " in the equation above. This is done to gain the frequency independence by increasing the number of unknowns in the dynamic equation. In this case, the number of dissipation coordinates needs to be equal to one because the number of perturbing terms is assumed to be one. Therefore, the dissipation coordinates for two types of damping material are:

$$
\begin{gathered}
\hat{\mathbf{Z}}_{\mathbf{1}}(s)=\frac{b_{1}}{s+b_{1}} \mathbf{X}(s) \Rightarrow \dot{\hat{\mathbf{z}}}_{\mathbf{1}}+b_{1} \hat{\mathbf{z}}_{\mathbf{1}}=b_{1} \mathbf{X} \\
\hat{\mathbf{Z}}_{2}(s)=\frac{b_{2}}{s+b_{2}} \mathbf{X}(s) \Rightarrow \dot{\hat{\mathbf{z}}}_{\mathbf{2}}+b_{2} \hat{\mathbf{z}}_{\mathbf{2}}=b_{2} \mathbf{x}
\end{gathered}
$$

Combine Equation (4.28) and (4.29) to form a simultaneous equation system and take the Inverse Laplace Transform: 


$$
\left\{\begin{array}{l}
\mathbf{M} \ddot{\mathbf{x}}+\left[\mathbf{K}_{\mathrm{e}}+G_{1}^{\infty}\left(1+a_{1}\right) \mathbf{K}_{\mathbf{v} 1}+G_{2}^{\infty}\left(1+a_{2}\right) \mathbf{K}_{\mathrm{v} 2}\right] \mathbf{x}-G_{1}^{\infty} a_{1} \mathbf{K}_{\mathrm{v} 1} \hat{\mathbf{z}}_{1}-G_{2}^{\infty} a_{2} \mathbf{K}_{\mathrm{v} 2} \hat{\mathbf{z}}_{2}=\mathbf{f}(t) \\
-b_{1} \mathbf{x}+\dot{\hat{\mathbf{z}}}_{1}+b_{1} \hat{\mathbf{z}}_{1}=0 \\
-b_{2} \mathbf{x}+\dot{\hat{\mathbf{z}}}_{\mathbf{2}}+b_{2} \hat{\mathbf{z}}_{2}=0
\end{array}\right.
$$

Rewrite the equation system as the matrix form:

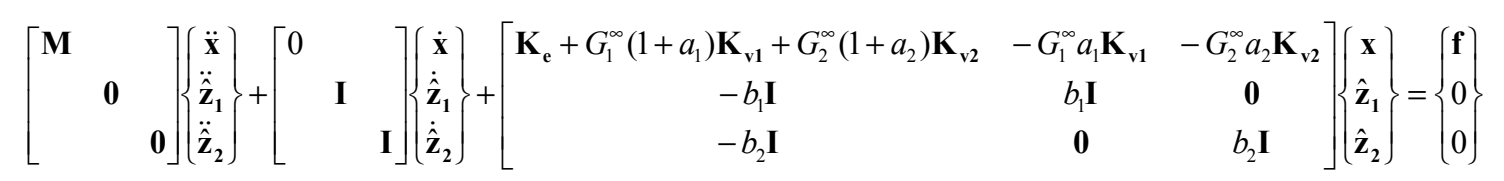

The stiffness matrix in the Equation (4.31) needs to be symmetric for further analysis according to the Maxwell Principle. Mathematical manipulation will be performed to obtain the equivalent symmetric matrix. The nomenclature applied to two different damping materials respectively is defined as:

$$
\begin{gathered}
\mathbf{K}_{\mathrm{v} 1}=\mathbf{R}_{\mathrm{v} 1} \Lambda_{\mathrm{v} 1} \mathbf{R}_{\mathrm{v} 1}^{\mathrm{T}} ; \mathbf{K}_{\mathrm{v} 2}=\mathbf{R}_{\mathrm{v} 2} \Lambda_{\mathrm{v} 2} \mathbf{R}_{\mathrm{v} 2}^{\mathrm{T}} \\
\mathbf{z}_{1}=\mathbf{R}_{\mathrm{v} 1}^{\mathrm{T}} \hat{\mathbf{z}}_{1} ; \mathbf{z}_{2}=\mathbf{R}_{\mathrm{v} 2}^{\mathrm{T}} \hat{\mathbf{z}}_{2} \Rightarrow \hat{\mathbf{z}}_{1}=\left[\mathbf{R}_{\mathrm{v} 1}^{\mathrm{T}}\right]^{-1} \mathbf{z}_{1} ; \hat{\mathbf{z}}_{2}=\left[\mathbf{R}_{\mathrm{v} 2}^{\mathrm{T}}\right]^{-1}
\end{gathered}
$$

where $\Lambda_{\mathrm{vi}}$ and $\mathrm{R}_{\mathrm{vi}}$ are the diagonal eigenvalue and the eigenvector matrix of the damping stiffness matrix as $\mathrm{K}_{\mathrm{vi}}$.

The auxiliary equation obtained from the introduction of the dissipation coordinate will be transformed to form the symmetric stiffness matrix of Equation (4.31): 


$$
\frac{1}{b_{1}} \dot{\mathbf{z}}_{\mathbf{1}}+\hat{\mathbf{z}}_{\mathbf{1}}-\mathbf{x}=0 \Rightarrow \underbrace{\frac{1}{b_{1}}\left[\mathbf{R}_{\mathrm{v} 1}^{\mathrm{T}}\right]^{-1} \dot{\mathbf{z}}_{\mathbf{1}}+\left[\mathbf{R}_{\mathrm{v} 1}^{\mathrm{T}}\right]^{-1} \mathbf{z}_{\mathbf{1}}-\mathbf{x}=0}_{\times G_{1}^{\infty} a_{1} \boldsymbol{\Lambda}_{\mathrm{v} 1} \mathbf{R}_{\mathrm{v} 1}^{\mathrm{T}}} \Rightarrow G_{1}^{\infty} \frac{a_{1}}{b_{1}} \mathbf{\Lambda}_{\mathrm{v} 1} \dot{\mathbf{z}}_{\mathbf{1}}+G_{1}^{\infty} a_{1} \boldsymbol{\Lambda}_{\mathrm{v} 1} \mathbf{z}_{\mathbf{1}}-G_{1}^{\infty} a_{1} \boldsymbol{\Lambda}_{\mathrm{v} 1} \mathbf{R}_{\mathrm{v} 1}^{\mathrm{T}} \mathbf{x}=0
$$

Similarly,

$$
\frac{1}{b_{2}} \dot{\mathbf{z}}_{2}+\hat{\mathbf{z}}_{2}-\mathbf{x}=0 \Rightarrow G_{2}^{\infty} \frac{a_{2}}{b_{2}} \boldsymbol{\Lambda}_{\mathrm{v} 2} \dot{\mathbf{z}}_{2}+G_{2}^{\infty} a_{2} \boldsymbol{\Lambda}_{\mathrm{v} 2} \mathbf{z}_{2}-G_{2}^{\infty} a_{2} \boldsymbol{\Lambda}_{\mathrm{v} 2} \mathbf{R}_{\mathrm{v} 2}^{\mathrm{T}} \mathbf{x}=0
$$

Now, with the Equation (4.33), the simultaneous equation system can be rewritten with the symmetric stiffness matrix as:

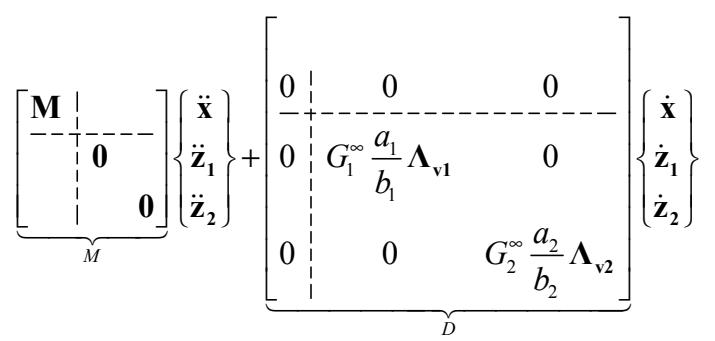

$$
\begin{aligned}
& \underbrace{\left[\begin{array}{c:cc}
\mathbf{K}_{\mathrm{e}}+G_{1}^{\infty}\left(1+a_{1}\right) \mathbf{K}_{\mathrm{v} 1}+G_{2}^{\infty}\left(1+a_{2}\right) \mathbf{K}_{\mathrm{v} 2} & -G_{1}^{\infty} a_{1} \mathbf{R}_{\mathrm{v} 1} \mathbf{\Lambda}_{\mathrm{v} 1} & -G_{2}^{\infty} a_{2} \mathbf{R}_{\mathrm{v} 2} \boldsymbol{\Lambda}_{\mathrm{v} 2} \\
\hdashline-G_{1}^{\infty} a_{1} \boldsymbol{\Lambda}_{\mathrm{v} 1} \mathbf{R}_{\mathrm{v} 1}^{T} & G_{1}^{\infty} a_{1} \boldsymbol{\Lambda}_{\mathrm{v} 1} & \mathbf{0} \\
-G_{2}^{\infty} a_{2} \boldsymbol{\Lambda}_{\mathrm{v} 2} \mathbf{R}_{\mathrm{v} 2}^{T} & \mathbf{0} & G_{2}^{\infty} a_{2} \mathbf{\Lambda}_{\mathrm{v} 2}
\end{array}\right]}_{K}\left\{\begin{array}{l}
\mathbf{x} \\
\mathbf{z}_{1} \\
\mathbf{z}_{2}
\end{array}\right\}=\left\{\begin{array}{l}
\mathbf{f} \\
0 \\
0
\end{array}\right\}
\end{aligned}
$$

The following additional nomenclature needs to be used in order to simplify the Equation 4.34 .

$$
\begin{gathered}
G_{1}^{\infty} \boldsymbol{\Lambda}_{\mathrm{v} 1}=\boldsymbol{\Lambda}_{\mathbf{1}} ; G_{2}^{\infty} \boldsymbol{\Lambda}_{\mathrm{v} 2}=\boldsymbol{\Lambda}_{\mathbf{2}} \\
G_{1}^{\infty} \mathbf{R}_{\mathrm{v} 1} \boldsymbol{\Lambda}_{\mathrm{v} 1}=\mathbf{R}_{\mathbf{1}} \Rightarrow \mathbf{R}_{1}^{T}=G_{1}^{\infty} \boldsymbol{\Lambda}_{\mathrm{v} 1}^{\mathrm{T}} \mathbf{R}_{\mathrm{v} 1}^{\mathrm{T}} \\
G_{2}^{\infty} \mathbf{R}_{\mathrm{v} \mathbf{2}} \boldsymbol{\Lambda}_{\mathrm{v} 2}=\mathbf{R}_{\mathbf{2}} \Rightarrow \mathbf{R}_{2}^{\mathrm{T}}=G_{2}^{\infty} \boldsymbol{\Lambda}_{\mathrm{v} 2}^{\mathrm{T}} \mathbf{R}_{\mathrm{v} 2}^{\mathrm{T}}
\end{gathered}
$$

Thus, the dynamic equation applying Biot damping model (assuming one perturbing term) with two different damping materials can be manipulated as the symmetric form: 


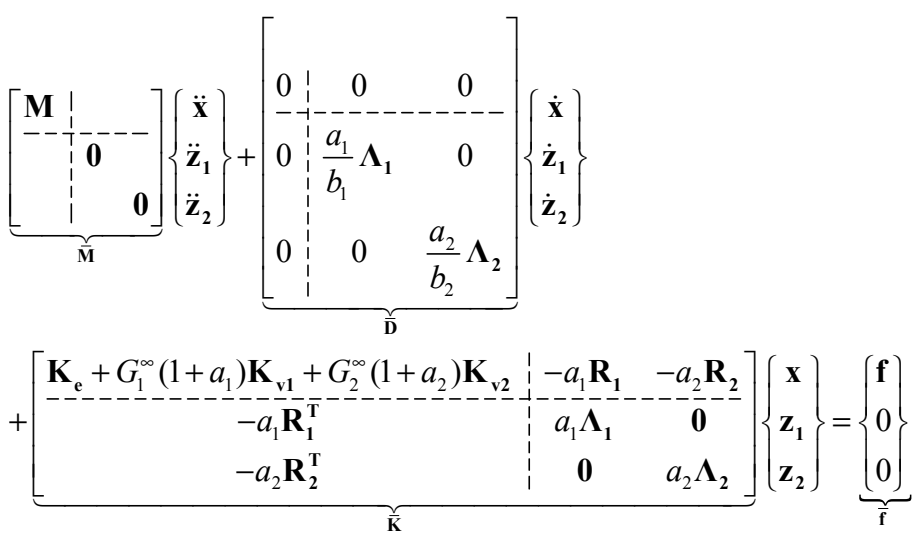

If the number of perturbing terms is extended from one to infinite, defining:

$a_{11} \ldots a_{1 m}, b_{11} \ldots b_{1 m}$ and $z_{11} \ldots z_{1 m}$ as $m$ terms of Biot parameters and the dissipative coordinates respectively for the $1^{\text {st }}$ viscoelastic material;

Similarly, $a_{21} \ldots a_{2 n}, b_{21} \ldots b_{2 n}$ and $z_{21} \ldots z_{2 n}$ as $n$ terms of Biot parameters and the dissipation coordinates respectively for $2^{\text {nd }}$ viscoelastic material;

Therefore, the dynamic equation with $\mathrm{m}$ terms of Biot parameters for $1^{\text {st }}$ viscoelastic material and $\mathrm{n}$ terms of $2^{\text {nd }}$ will become as follows: 

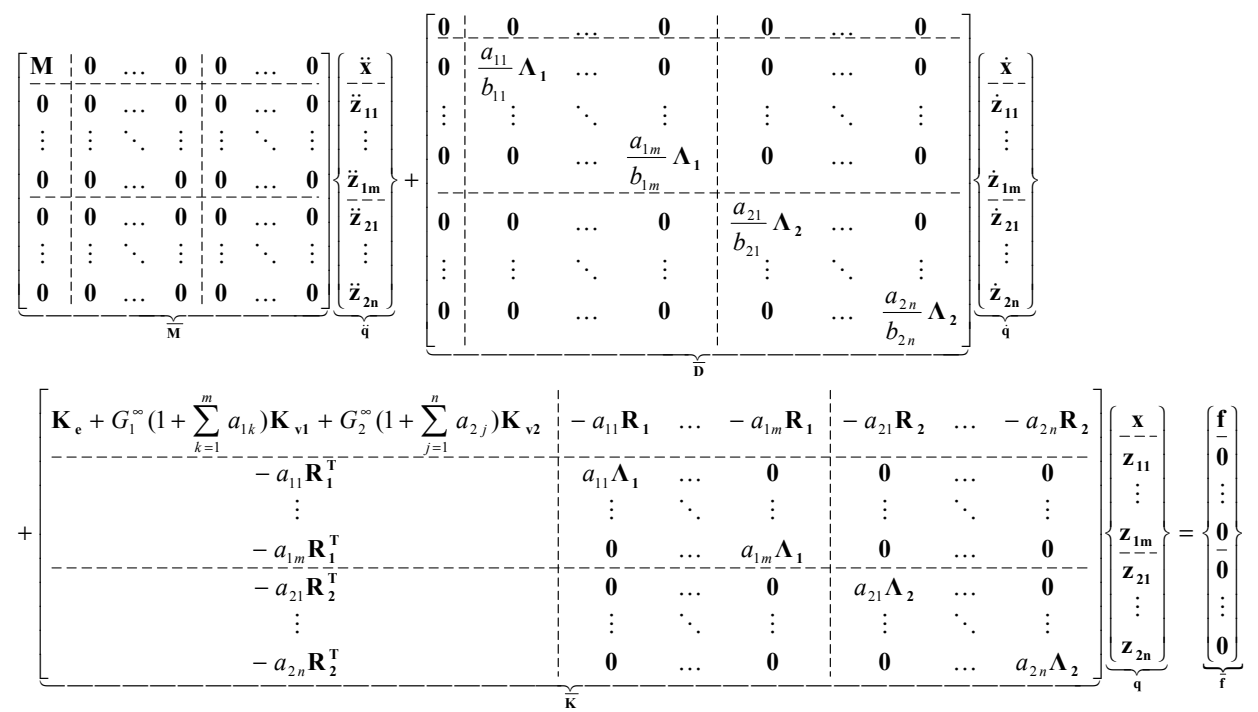

Supposing $\mathrm{N}$ is the number of DOF for the mass, stiffness and damping matrix, the dimension of each square matrix in the equation above is $(m+n+1) N$, and the $q$ vector stands for the unknowns, especially the $\mathrm{x}$ vector as the system displacement response.

In sum, by introducing the Biot damping model to apply the viscoelastic properties of a descritized system, a non-linear ODE (Equation (4.24)) is transformed to a linear 2nd order ODE (Equation37). Solving the eigenvalue problem based on Equation (4.37) determines the vibration characteristics of the system such as: natural frequency, loss factor and complex mode shape.

Additionally, Equation (4.37) may easily be extended from 2 types of viscoelastic 
material to infinite types in the same manner using Equation (4.24) through Equation (4.37). Thus, the dimensions of the square matrix in the Biot dynamic equation with infinite types of viscoelastic material is: $(1+\Sigma$ number of Biot terms for all the materials) $\mathrm{N}$. 


\subsection{Decoupling Transformation and Dynamic Solution}

The procedure of finding the complex eigenvalue and time-domain transient response will be established with respect to the vibration problem for a multiple-layer viscoelastic damping structure in this section. In general, if the damping matrix $\boldsymbol{C}$ has the following proportional relationship with mass matrix $\boldsymbol{M}$ and stiffness matrix $\boldsymbol{K}$,

$$
\mathbf{C}=\alpha \mathbf{M}+\beta \mathbf{K}
$$

the modal damping matrix will be diagonal, in which $\alpha$ and $\beta$ are the proportional damping constants. Obviously, the damping matrix $\overline{\mathbf{D}}$ in Equation (4.37) does not have the proportional relationship with the mass and stiffness matrix. Therefore, the decoupling transformation is needed for Equation (4.37) to construct a first-order state equation by introducing the auxiliary equation $\mathbf{M} \dot{\mathbf{q}}-\mathbf{M} \dot{\mathbf{q}}=\mathbf{0}$ as:

$$
\mathbf{A} \dot{\mathbf{y}}+\mathbf{B y}=\hat{\mathbf{f}}
$$

where

$$
\begin{gathered}
\mathbf{A}=\left[\begin{array}{cc}
\overline{\mathbf{D}} & \overline{\mathbf{M}} \\
\overline{\mathbf{M}} & \mathbf{0}
\end{array}\right] ; \mathbf{B}=\left[\begin{array}{cc}
\overline{\mathbf{K}} & \mathbf{0} \\
\mathbf{0} & -\overline{\mathbf{M}}
\end{array}\right] ; \\
\mathbf{y}=\left\{\begin{array}{l}
\mathbf{q} \\
\dot{\mathbf{q}}
\end{array}\right\} ; \hat{\mathbf{f}}=\left\{\begin{array}{l}
\overline{\mathbf{f}} \\
\mathbf{0}
\end{array}\right\}
\end{gathered}
$$


If $\mathrm{N}$ is the number of DOF in the $\overline{\mathbf{M}}, \overline{\mathbf{D}}$ and $\overline{\mathbf{K}}$ matrices, the DOF of $\boldsymbol{A}$ and $\boldsymbol{B}$ matrix will be $2 \mathrm{~N}$.

Firstly, the free vibration of Equation (4.39) will be considered. Assuming $\hat{\mathbf{f}}=0$, the following form of solution yields:

$$
(\mathbf{A} \lambda+\mathbf{B y}) \boldsymbol{\Phi}=\mathbf{0}
$$

or

$$
(\mathbf{A} \lambda+\mathbf{B y})\left\{\begin{array}{c}
\Psi \\
\Psi \lambda
\end{array}\right\}=\mathbf{0}
$$

Where $\lambda$ matrix stands for $2 \mathrm{~N}$ complex conjugate eigenvalues including the natural frequencies and loss factors information:

$$
\left[\begin{array}{lll}
\ddots & & \\
& \lambda & \\
& & \ddots
\end{array}\right]=\left[\begin{array}{lllll}
\lambda_{1} & & & & \\
& \lambda_{1}^{*} & & 0 & \\
& & \ddots & & \\
& 0 & & \lambda_{N} & \\
& & & & \lambda_{N}^{*}
\end{array}\right]
$$

It must be noted that zero items will appear in the eigenvalue matrix if the damping matrix $\overline{\mathbf{D}}$ is not fully ranked. The mode shape vector $\boldsymbol{\Psi}$ for the vector $\boldsymbol{q}$ can be extracted from the eigenvector matrix $\boldsymbol{\Phi}$ with respect to the vector $\boldsymbol{y}$ :

$$
[\Phi]=\left\{\begin{array}{ccccc}
{[\Psi]_{1}} & {[\Psi]_{1}^{*}} & \cdots & {[\Psi]_{N}} & {[\Psi]_{N}^{*}} \\
\lambda_{1}[\Psi]_{1} & \lambda_{1}^{*}[\Psi]_{1}^{*} & \cdots & \lambda_{N}[\Psi]_{N} & \lambda_{N}^{*}[\Psi]_{N}^{*}
\end{array}\right\}
$$


In addition, the Equation (4.43) can be numerically solved by $\lambda\{\Psi\}=-[\mathbf{A}]^{-1}[\mathbf{B}]\{\Psi\}$ using mathematical software package such as MATLAB or Mathematica.

Secondly, the forced vibration solution of Equation (4.39) in the time domain will be discussed. Supposing $\hat{\mathbf{f}}=\left\{\begin{array}{l}\mathbf{F} \\ \mathbf{0}\end{array}\right\}$, the variable substitution can be made by assuming: $\mathbf{y}=\boldsymbol{\Phi} \mathbf{p}$, converting the state-space equation from the time space to modal space. The Equation (4.39) yields by left-multiplying of $\boldsymbol{\Phi}^{T}$ with the substitution of y:

$$
\Phi^{\mathrm{T}} \mathbf{A} \Phi \dot{\mathbf{p}}+\boldsymbol{\Phi}^{\mathrm{T}} \mathbf{B} \boldsymbol{\Phi} \mathbf{p}=\Phi^{\mathrm{T}} \hat{\mathbf{f}}
$$

The diagonal modal mass and stiffness matrices are:

$$
\boldsymbol{\Phi}^{\mathbf{T}} \mathbf{A} \boldsymbol{\Phi}=\left[\begin{array}{lll}
\ddots & & \\
& M_{p} & \\
& & \ddots
\end{array}\right] ; \boldsymbol{\Phi}^{\mathbf{T}} \mathbf{B} \boldsymbol{\Phi}=\left[\begin{array}{lll}
\ddots & & \\
& K_{p} & \\
& & \ddots
\end{array}\right]
$$

Then rewrite the Equation (4.46) with the diagonal mass and stiffness matrices:

$$
\left[\begin{array}{lll}
\ddots & & \\
& M_{p} & \\
& & \ddots
\end{array}\right] \dot{\mathbf{p}}+\left[\begin{array}{lll}
\ddots & & \\
& K_{p} & \\
& & \ddots
\end{array}\right] \mathbf{p}=\boldsymbol{\Phi}^{\mathrm{T}} \widehat{\mathbf{f}}
$$

Due to the relationship: $\frac{\mathbf{K}_{\mathbf{p} i}}{\mathbf{M}_{\mathbf{p} i}}=-\lambda_{i}$, the above equation can be converted to $2 \mathrm{~N}$ numbers of $1^{\text {st }}$ order linear ODE: 


$$
\dot{p}_{i}-\lambda_{i} p_{i}=\frac{1}{M_{p i}} \boldsymbol{\Psi}_{\mathbf{i}}{ }^{\mathrm{T}} \hat{\mathbf{f}}_{i}
$$

The steady-state solution of the above equation when the system is at rest can be expressed as an integral equation, and is also equivalent to the convolution in the time domain between the arbitrary force term $\frac{1}{M_{p i}} \Psi_{i}^{T} \hat{f}_{i}(t)$ and dissipation term $e^{-\lambda_{i} t}$ :

$$
p_{i}=\frac{1}{M_{p i}} \int_{0}^{t} \boldsymbol{\Psi}_{\mathbf{i}}{ }^{\mathrm{T}} \widehat{\mathbf{f}}_{\mathbf{i}}(\boldsymbol{\tau}) \bullet e^{-\lambda_{i}(t-\tau)} d \tau=\frac{1}{M_{p i}} \boldsymbol{\Psi}_{\mathbf{i}}{ }^{T} \widehat{\mathbf{f}}_{\mathbf{i}}(\mathbf{t}) \otimes e^{-\lambda_{i} t}
$$

Compared with the conventional integral method, it is easier to find the engineering value using this convolution method. The conventional method requires the exact mathematical expression of this force in the time domain. The convolution method, on the other hand, only requires the arbitrary force plot in the time domain.. Thus the modal superposition method can be established by:

$$
\mathbf{y}=\left\{\begin{array}{c}
\mathbf{q} \\
\dot{\mathbf{q}}
\end{array}\right\}=\boldsymbol{\Phi} \mathbf{p}=\sum_{i=1}^{2 N} \boldsymbol{\Psi}_{\mathbf{i}} p_{i}
$$

Considering the issue of curve-fitting range, it is necessary to sum up the modes only in the selected range, which has fewer than $2 \mathrm{~N}$ modes. In summary, the 
complex mode shape vector $\boldsymbol{\Psi}_{\text {and }}$ complex eigenvalue $\boldsymbol{\lambda}$ in each mode are key factors in determining the time-domain response for a viscoelastic damping structure.

Thirdly, the FRF in the frequency domain can be easily determined through the complex conjugate eigenvalue matrix $\lambda$, eigenvector matrix $\Phi$ and the modal mass matrix $\mathrm{M}_{\mathrm{p}}$ from Equation (4.47). The modal scaling factor matrix can be calculated through:

$$
\left[\begin{array}{lll}
\ddots & & \\
& \mathbf{Q} & \\
& & \ddots
\end{array}\right]=\left[\begin{array}{lll}
\ddots & & \\
& \mathbf{M}_{\mathbf{p}} & \\
& & \ddots
\end{array}\right]^{-1}
$$

Thus the FRF can be established through the modal parameters. The FRF can be also expressed in partial fraction form in terms of the residue vector and system poles as follows:

$$
[H(j \omega)]=\frac{X(j \omega)}{F(j \omega)}=\sum_{i=1}^{N}\left[\frac{Q_{i} \boldsymbol{\Psi}_{\mathbf{i}} \boldsymbol{\Psi}_{\mathbf{i}}^{\mathbf{T}}}{\left(j \omega-\lambda_{i}\right)}+\frac{Q_{i} \boldsymbol{\Psi}_{\mathbf{i}}^{*} \boldsymbol{\Psi}_{\mathbf{i}}^{* \mathbf{T}}}{\left(j \omega-\lambda_{i}^{*}\right)}\right]
$$

Meanwhile, the system velocity can be figured out from the above equation by simple Fourier Transformation. By doing so, the vibration problem can be extended to an FRF-based acoustical problem and the conjunction of these two analyses is the 
particle velocities information calculated by following equation:

$$
\dot{X}(j \omega)=j \omega[H(j \omega)] F(j \omega)=j \omega[F(j \omega)] \cdot \sum_{i=1}^{N}\left[\frac{Q_{i} \boldsymbol{\Psi}_{\mathbf{i}} \mathbf{\Psi}_{\mathbf{i}}^{\mathrm{T}}}{\left(j \omega-\lambda_{i}\right)}+\frac{Q_{i} \boldsymbol{\Psi}_{\mathbf{i}}^{*} \boldsymbol{\Psi}_{\mathbf{i}}^{* \mathbf{T}}}{\left(j \omega-\lambda_{i}^{*}\right)}\right]
$$




\subsection{Dimension Reduction Technique}

The accomplishment of dimension reduction technique $[55,91,92]$ is advantageous for increasing the computational efficiency especially for the numerical analysis of the Biot damping model. In equation (4.42), two different viscoelastic materials are referred with perturbing terms of $\mathrm{m}$ and $\mathrm{n}$ respectively. It is found that by applying the Biot damping model to these materials, the original mass, damping and stiffness matrix with N DOF now sharply increases to $(\mathrm{m}+\mathrm{n}+1) \mathrm{N}$ DOF. However, a large number of zero items are found in the equivalent mass matrix $\overline{\mathbf{M}}$ due to the introduction of the dissipative coordinates of the Biot damping model. Thus, these zero items can be the major source of this reduction task.

With the equivalent mass, damping and stiffness matrix $(\overline{\mathbf{M}}, \overline{\mathbf{D}}$ and $\overline{\mathbf{K}})$, the Equation 4.42 can be expanded as the matrix form: 


$$
\mathbf{A}=\left[\begin{array}{ccccccccccccccc}
\mathbf{0} & \mathbf{0} & \ldots & \mathbf{0} & \mathbf{0} & \ldots & \mathbf{0} & \mathbf{M} & \mathbf{0} & \ldots & \mathbf{0} & \mathbf{0} & \ldots & \mathbf{0} \\
\mathbf{0} & \frac{a_{11}}{b_{11}} \boldsymbol{\Lambda}_{1} & \ldots & \mathbf{0} & \mathbf{0} & \ldots & \mathbf{0} & \mathbf{0} & \mathbf{0} & \ldots & \mathbf{0} & \mathbf{0} & \ldots & \mathbf{0} \\
\vdots & \vdots & \ddots & \vdots & \vdots & \ddots & \vdots & \vdots & \vdots & \ddots & \vdots & \vdots & \ddots & \vdots \\
\mathbf{0} & \mathbf{0} & \ldots & \frac{a_{1 m}}{b_{1 m}} \boldsymbol{\Lambda}_{1} & \mathbf{0} & \ldots & \mathbf{0} & \mathbf{0} & \mathbf{0} & \ldots & \mathbf{0} & \mathbf{0} & \ldots & \mathbf{0} \\
\mathbf{0} & \mathbf{0} & \ldots & \mathbf{0} & \frac{a_{21}}{b_{21}} \boldsymbol{\Lambda}_{2} & \ldots & \mathbf{0} & \mathbf{0} & \mathbf{0} & \ldots & \mathbf{0} & \mathbf{0} & \ldots & \mathbf{0} \\
\vdots & \vdots & \ddots & \vdots & \vdots & \ddots & \vdots & \vdots & \vdots & \ddots & \vdots & \vdots & \ddots & \vdots \\
\mathbf{0} & \mathbf{0} & \ldots & \mathbf{0} & \mathbf{0} & \ldots & \frac{a_{2 n}}{\boldsymbol{\Lambda}_{2 n}} & \mathbf{0} & \mathbf{0} & \ldots & \mathbf{0} & \mathbf{0} & \ldots & \mathbf{0} \\
\hdashline \mathbf{0} & \mathbf{0} & \ldots & \mathbf{0} & \mathbf{0} & \ldots & \mathbf{0} & \mathbf{0} & \mathbf{0} & \ldots & \mathbf{0} & \mathbf{0} & \ldots & \mathbf{0} \\
\vdots & \vdots & \ddots & \vdots & \vdots & \ddots & \vdots & \vdots & \vdots & \ddots & \vdots & \vdots & \ddots & \vdots \\
\mathbf{0} & \mathbf{0} & \ldots & \mathbf{0} & \mathbf{0} & \ldots & \mathbf{0} & \mathbf{0} & \mathbf{0} & \ldots & \mathbf{0} & \mathbf{0} & \ldots & \mathbf{0} \\
\mathbf{0} & \mathbf{0} & \ldots & \mathbf{0} & \mathbf{0} & \ldots & \mathbf{0} & \mathbf{0} & \mathbf{0} & \ldots & \mathbf{0} & \mathbf{0} & \ldots & \mathbf{0} \\
\vdots & \vdots & \ddots & \vdots & \vdots & \ddots & \vdots & \vdots & \vdots & \ddots & \vdots & \vdots & \ddots & \vdots \\
\mathbf{0} & \mathbf{0} & \ldots & \mathbf{0} & \mathbf{0} & \ldots & \mathbf{0} & \mathbf{0} & \mathbf{0} & \ldots & \mathbf{0} & \mathbf{0} & \ldots & \mathbf{0}
\end{array}\right]
$$

$$
\mathbf{B}=\left[\begin{array}{cccccccc:cccccc}
\mathbf{K}_{\mathbf{e}}+G_{1}^{\infty}\left(1+\sum_{k=1}^{m} a_{1 k}\right) \mathbf{K}_{\mathrm{v1}}+G_{2}^{\infty}\left(1+\sum_{j=1}^{n} a_{2 j}\right) \mathbf{K}_{\mathrm{v2}} & -a_{1} \mathbf{R}_{1} & \ldots & -a_{1 m} \mathbf{R}_{1} & -a_{21} \mathbf{R}_{2} & \ldots & -a_{2 n} \mathbf{R}_{2} & \mathbf{0} & \mathbf{0} & \ldots & \mathbf{0} & \mathbf{0} & \ldots & \mathbf{0} \\
-a_{11} \mathbf{R}_{1}^{\mathrm{T}} & a_{11} \mathbf{\Lambda}_{1} & \ldots & \mathbf{0} & \mathbf{0} & \ldots & \mathbf{0} & \mathbf{0} & \mathbf{0} & \ldots & \mathbf{0} & \mathbf{0} & \ldots & \mathbf{0} \\
\vdots & \vdots & \ddots & \vdots & \vdots & \ddots & \vdots & \vdots & \vdots & \ddots & \vdots & \vdots & \ddots & \vdots \\
-a_{1 m} \mathbf{R}_{1}^{\mathrm{T}} & \mathbf{0} & \ldots & a_{1 m} \mathbf{\Lambda}_{1} & \mathbf{0} & \ldots & \mathbf{0} & \mathbf{0} & \mathbf{0} & \ldots & \mathbf{0} & \mathbf{0} & \ldots & \mathbf{0} \\
-a_{21} \mathbf{R}_{2}^{\mathrm{T}} & \mathbf{0} & \ldots & \mathbf{0} & a_{21} \mathbf{\Lambda}_{2} & \ldots & \mathbf{0} & \mathbf{0} & \mathbf{0} & \ldots & \mathbf{0} & \mathbf{0} & \ldots & \mathbf{0} \\
\vdots & \vdots & \ddots & \vdots & \vdots & \ddots & \vdots & \vdots & \vdots & \ddots & \vdots & \vdots & \ddots & \vdots \\
-a_{2 n} \mathbf{R}_{2}^{\mathrm{T}} & \mathbf{0} & \ldots & \mathbf{0} & \mathbf{0} & \ldots & a_{2 n} \mathbf{\Lambda}_{2} & \mathbf{0} & \mathbf{0} & \ldots & \mathbf{0} & \mathbf{0} & \ldots & \mathbf{0} \\
\mathbf{0} & \mathbf{0} & \ldots & \mathbf{0} & \mathbf{0} & \ldots & \mathbf{0} & -\mathbf{M} & \mathbf{0} & \ldots & \mathbf{0} & \mathbf{0} & \ldots & \mathbf{0} \\
\hdashline \mathbf{0} & \mathbf{0} & \ldots & \mathbf{0} & \mathbf{0} & \ldots & \mathbf{0} & \mathbf{0} & \mathbf{0} & \ldots & \mathbf{0} & \mathbf{0} & \ldots & \mathbf{0} \\
\vdots & \vdots & \ddots & \vdots & \vdots & \ddots & \vdots & \vdots & \vdots & \ddots & \vdots & \vdots & \ddots & \vdots \\
\mathbf{0} & \mathbf{0} & \ldots & \mathbf{0} & \mathbf{0} & \ldots & \mathbf{0} & \mathbf{0} & \mathbf{0} & \ldots & \mathbf{0} & \mathbf{0} & \ldots & \mathbf{0} \\
\mathbf{0} & \mathbf{0} & \ldots & \mathbf{0} & \mathbf{0} & \ldots & \mathbf{0} & \mathbf{0} & \mathbf{0} & \ldots & \mathbf{0} & \mathbf{0} & \ldots & \mathbf{0} \\
\vdots & \vdots & \ddots & \vdots & \vdots & \ddots & \vdots & \vdots & \vdots & \ddots & \vdots & \vdots & \ddots & \vdots \\
\mathbf{0} & \mathbf{0} & \ldots & \mathbf{0} & \mathbf{0} & \ldots & \mathbf{0} & \mathbf{0} & \mathbf{0} & \ldots & \mathbf{0} & \mathbf{0} & \ldots & \mathbf{0}
\end{array}\right]
$$

$$
\hat{\mathbf{f}}=\left\{\begin{array}{llllllll:llllll}
\mathbf{f} & \mathbf{0} & \cdots & \mathbf{0} & \mathbf{0} & \cdots & \mathbf{0} & \mathbf{0} & \mathbf{0} & \cdots & \mathbf{0} & \mathbf{0} & \cdots & \mathbf{0}
\end{array}\right\}^{T}
$$




$$
\mathbf{y}=\{\mathbf{q} \mid \dot{\mathbf{x}}\}
$$

As shown in Equation (4.53-4.54), the dotted lines separate the zero subsets from original matrix forms. By this dimension reduction technique, all the zero elements can be easily removed without any hampering of the dynamic behaviors and the dimension of each matrix is considerably reduced from $2 \mathrm{~N}(1+\mathrm{m}+\mathrm{n})$ to $\mathrm{N}(2+\mathrm{m}+\mathrm{n})$ by removing these zero subsets. This method can be named as "zero-Subset" reduction technique.

Another source of dimension reduction is from the original damping matrix $\boldsymbol{C}$. Because of different numerical modeling techniques, sometimes the damping matrix $\boldsymbol{C}$ cannot be fully ranked, thus the eigenvalue of this matrix $\lambda$ includes several zero items with empty columns in the corresponding eigenvector matrix $\boldsymbol{R}$. Two damping matrices of identical rank are considered, with the number of eigenvalues represented by $\mathrm{d}$. When the rank of the damping matrix is subtracted from $\mathrm{N}$ (DOF of the damping matrix), the resulting dimension of the equivalent mass, damping and stiffness matrix $\overline{\mathbf{M}}, \overline{\mathbf{D}}, \overline{\mathbf{K}}$ is $(\mathrm{N}-\mathrm{d})(\mathrm{m}+\mathrm{n})+\mathrm{N}$. "zero-Eigenvalue" can be the name of this technique. 
By applying the decoupling transformation (discussed in Chapter 4.3), the dimension of the system state-equation increased from $(\mathrm{N}-\mathrm{d})(\mathrm{m}+\mathrm{n})+\mathrm{N}$ to $(\mathrm{N}-\mathrm{d})(\mathrm{m}+\mathrm{n})+2 \mathrm{~N}$ by combining the zero-subset reduction technique. Therefore, the total dimension reduction is $(\mathrm{m}+\mathrm{n})(\mathrm{N}+\mathrm{d})$ DOFs compared between the dimension without using any reduction technique and the dimension using zero-Subset and zero-Eigenvalue methods together. The following flow-chart visualizes the dimension difference with and without the dimension reduction technique.

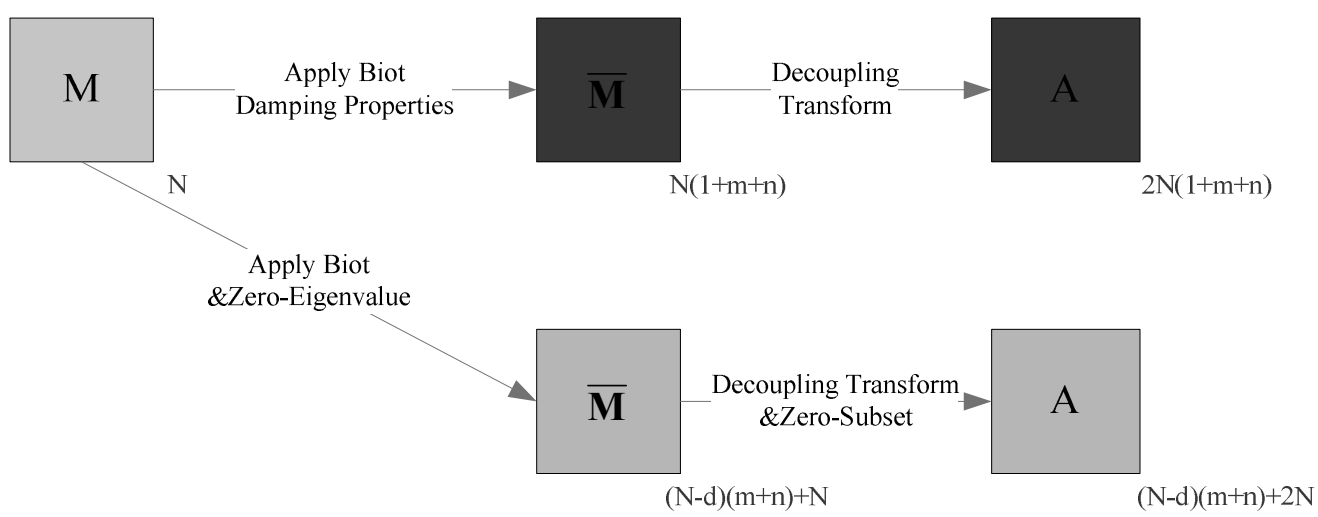

Figure 4.5 Comparison of the matrix dimension with/without the dimension reduction 


\subsection{Parametric Determination of the Biot Damping Model}

The background of damping mechanism is discussed in Chapter 2.2.2. The proper use of curve-fitting technique to provide the accurate Biot constants to the Biot dynamic equation is important. Generally speaking, the dynamic characteristics of the viscoelastic materials can be obtained from an experimental method. It is known that curve-fitting technique is normally employed to establish the connection between the experiment and the mathematical damping models. In this section, the non-linear curve-fitting procedure of the complex shear modulus in the frequency domain is converted into a non-linear constrained optimization problem.

Recalling the Equation (2.11) from Chapter 2.2.2, the complex shear modulus with the Biot form in the frequency domain is:

$$
s \widetilde{G}(j \omega)=G^{\infty}\left[1+\sum_{i=1}^{N} \frac{a_{i} \varpi^{2}}{b_{i}^{2}+\varpi^{2}}\right]+j G^{\infty}\left[1+\sum_{i=1}^{N} \frac{a_{i} b_{i} \varpi}{b_{i}^{2}+\varpi^{2}}\right]
$$

The Biot parameters including $G^{\infty}, \mathrm{a}_{\mathrm{i}}$ and $\mathrm{b}_{\mathrm{i}}$ are estimated from experimental data with the specific fit range of the frequency on both the real part and the imaginary part. The number of Biot perturbing items is given by $\mathrm{N}$, an integral constant, 
defining the capability of this numerical approximation. In general, variations in the temperature cause the changes in the Biot parameters in the equation above and one set of Biot parameters needs to be determined in each ambient temperature point independently.

Assuming: $x_{1}=G^{\infty} ; x_{2}=a_{1} ; x_{3}=b_{1} ; x_{4}=a_{2} ; x_{5}=b_{2} ; \cdots$

with the constraint condition: $x k \geq 0 ; k=1,2 \cdots, n u m$, the objective equation of this optimization problem can be expressed:

$$
\min _{x} F(x)=\sum_{j=1}^{P}\left|G_{j}^{*}(x)-G_{0 j}\right|^{2}
$$

in the above objective equation, $\mathrm{G}_{0 \mathrm{j}}$ stands for the complex shear modulus from the experimental data with $\mathrm{P}$ interested points, and the value of $\mathrm{P}$ needs to be bigger than the number of unknowns in this optimization problem.

In this research, the $3 \mathrm{M}$ ISD110 and 112 viscoelastic damping polymers are selected. Both materials have been proven to reduce vibration and acoustical responses in automobiles, disk drives and aircraft industries. The 3M ISD 110 has excellent damping performance at higher temperature from $40-105^{\circ} \mathrm{C}$ while the $3 \mathrm{M}$ ISD112 works ideally from $0-65^{\circ} \mathrm{C}$. The selection of temperature range in this research is 
$40-60^{\circ} \mathrm{C}$ to guarantee the good attenuation performance. The detailed specifications and experimental resulst can be referred to ref [93]. The Arrhenius equation from [94] provides the experimental data as well as damping properties such as the shear modulus and the loss factor for the 3M ISD 110 and 112 viscoelastic polymers. The sensitivity of the complex shear modulus for these viscoelastic materials varies with changes in the temperature and frequency. The universal form of empirical equation is listed as follows and the complex shear modulus is calculated through:

$$
G^{*}(\omega)=G_{c}(1+j \eta)
$$

In the above equation, the real part of shear modulus- $\mathrm{G}_{\mathrm{c}}$ is given by:

$$
\log \left(G_{c}\right)=\log (M L)+\frac{2 \times \log \left(\frac{M R O M}{M L}\right)}{1+\left(\frac{F R O M}{M L}\right)^{N}}
$$

the equation of the loss factor- $\eta$ is:

$$
\log (\eta)=\log (E T A F R)+\frac{C}{2}\left[\left(S_{L}+S_{H}\right) A+\left(S_{L}-S_{H}\right)\left(1-\sqrt{1+A^{2}}\right)\right]
$$

where A is:

$$
A=\frac{1}{C} \log \left(\frac{f r}{F R O L}\right)
$$

The reduced frequency caused by Arrhenius temperature shifting factor is:

$$
\log (f r)=\log (f)-\frac{12\left(T-T_{0}\right)}{291.66+T-T_{0}}
$$

In above equations: 
fr reduced frequency shifted by temperature effect $(\mathrm{Hz})$

f considered frequency $(\mathrm{Hz})$

$\mathrm{T}$ temperature (Centigrade degree)

and FROM, MROM, N, ML, ETAFROL, $\mathrm{S}_{\mathrm{L}}, \mathrm{S}_{\mathrm{H}}, \mathrm{FROL}, \mathrm{C}$, and $\mathrm{T}_{0}$ are the coefficients obtained from the following datasheet for the different types of viscoelastic materials.

Table 4.1 Arrhenius coefficients from ref [94]

\begin{tabular}{|c|c|c|c|c|c|c|c|c|c|c|c|}
\hline \multirow{3}{*}{$\begin{array}{l}\text { Damping } \\
\text { Material }\end{array}$} & \multirow{3}{*}{$\begin{array}{c}\text { Density } \\
\mathrm{kg} / \mathrm{m}^{3}\end{array}$} & \multirow{3}{*}{$\begin{array}{l}\mathrm{T}_{0} \\
\\
{ }_{C}\end{array}$} & \multicolumn{4}{|c|}{ Modulus Parameters } & \multicolumn{5}{|c|}{ Loss Factor Parameters } \\
\hline & & & FROM & MROM & $\mathrm{N}$ & ML & ETAFR & $\mathrm{S}_{\mathrm{L}}$ & $\mathrm{S}_{\mathrm{H}}$ & FROL & $\mathrm{C}$ \\
\hline & & & & $\mathrm{N} / \mathrm{m}^{2}$ & & $\mathrm{~N} / \mathrm{m}^{2}$ & & & & & \\
\hline $3 \mathrm{M}$ & 970 & 70 & $5 \mathrm{e} 3$ & $2 \mathrm{e} 6$ & 0.35 & $5.5 \mathrm{e} 4$ & 1.3 & 0.35 & -0.4 & $2 \mathrm{e} 3$ & 2 \\
\hline \multicolumn{12}{|l|}{ ISD-110 } \\
\hline $3 \mathrm{M}$ & 970 & 55 & $4 \mathrm{e} 5$ & $1.61 \mathrm{e} 7$ & 0.27 & $1.72 \mathrm{e}$ & 1.25 & 0.27 & -0.35 & $1.5 \mathrm{e} 5$ & 1.3 \\
\hline ISD-112 & & & & & 6 & 5 & & & & & \\
\hline
\end{tabular}

The following demonstrates the actual curve-fitting procedure. The 3M ISD-110/112 viscoelastic polymers are selected to verify the effectiveness of this procedure. The experimental data is given in the form of Arrhenius empirical equation. Assuming $\mathrm{T}$ is equal to $45^{\circ} \mathrm{C}$, and fit range is $500 \mathrm{~Hz}$, the complex shear modulus can be synthesized from the set of Arrhenius coefficients from the Table 4.1 and can then be calculated through Equation (4.60-4.63). Once the experimental data are given, the 
number of terms in Equation (4.57) needs to be determined to ensure the precision of this approximation. The commercial software package Auto2fit is used in this example because it is capable of curve-fitting the experimental data on both real and imaginary parts simultaneously. As the number of Biot terms $(\mathrm{N})$ is increased, the relative error between Arrhenius and curve-fitting result reduces; but, the amount of computational effort needed increases.. With the compromised number of Biot terms equal to six (ISD110) and four (ISD112), the result eventually lists in the Table 4.2 below. The curve-fitting data of ISD110/112 with different ambient temperatures is attached in the Appendix.

Table 4.2 Biot constants of $3 \mathrm{M}$ ISD- $110 / 11245^{\circ} \mathrm{C}$

\begin{tabular}{ccccccc}
\hline \hline & 3M-ISD110 & 3M-ISD112 & & 3M-ISD110 & 3M-ISD112 \\
$G^{\infty}$ & $55000(\mathrm{~Pa})$ & $172000(\mathrm{~Pa})$ & & & \\
$\mathrm{a} 1$ & 1.809517 & 5.699386303 & $\mathrm{~b} 1$ & 5.410993 & 4268.18097 \\
$\mathrm{a} 2$ & 14.53095 & 0.596843249 & $\mathrm{~b} 2$ & 1093.778 & 70.26089968 \\
$\mathrm{a} 3$ & 3.221535 & 1.000560485 & $\mathrm{~b} 3$ & 60.36544 & 501.5607814 \\
$\mathrm{a} 4$ & 52.01026 & 0.577694736 & $\mathrm{~b} 4$ & 4319.613 & 1.969150769 \\
a5 & 19768.22 & & $\mathrm{~b} 5$ & 2840958 & \\
a6 & 6.561162 & & $\mathrm{~b} 6$ & 298.0672 & \\
\hline \hline
\end{tabular}

The figure 4.6 and 4.7 below shows the real part and imaginary part of 3M-ISD 110 $45^{\circ} \mathrm{C}$ compared between the Arrhenius data and Curve-fitting data in this case: 


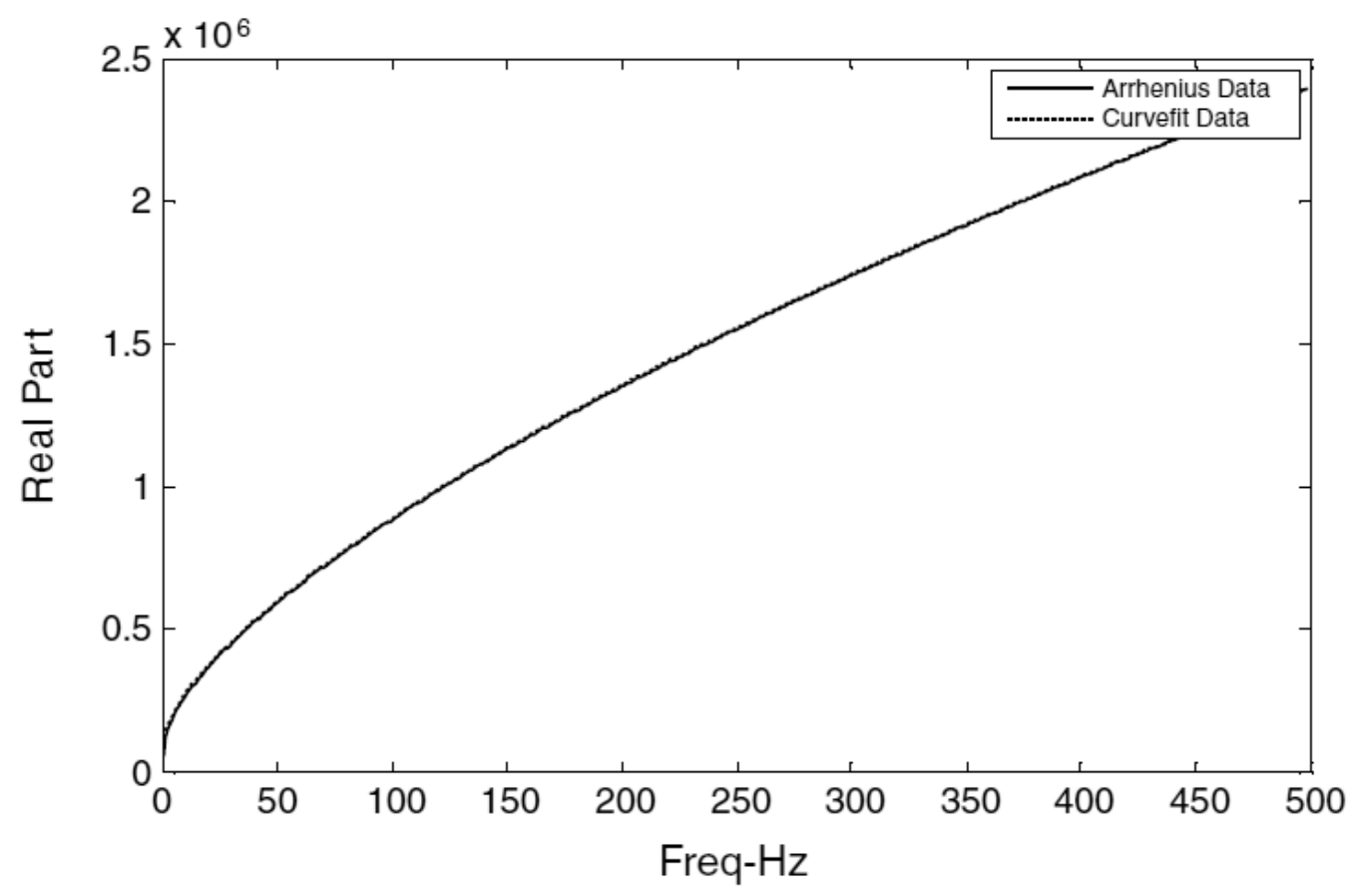

Figure 4.6 Real part comparison of ISD- $11045^{\circ} \mathrm{C}$

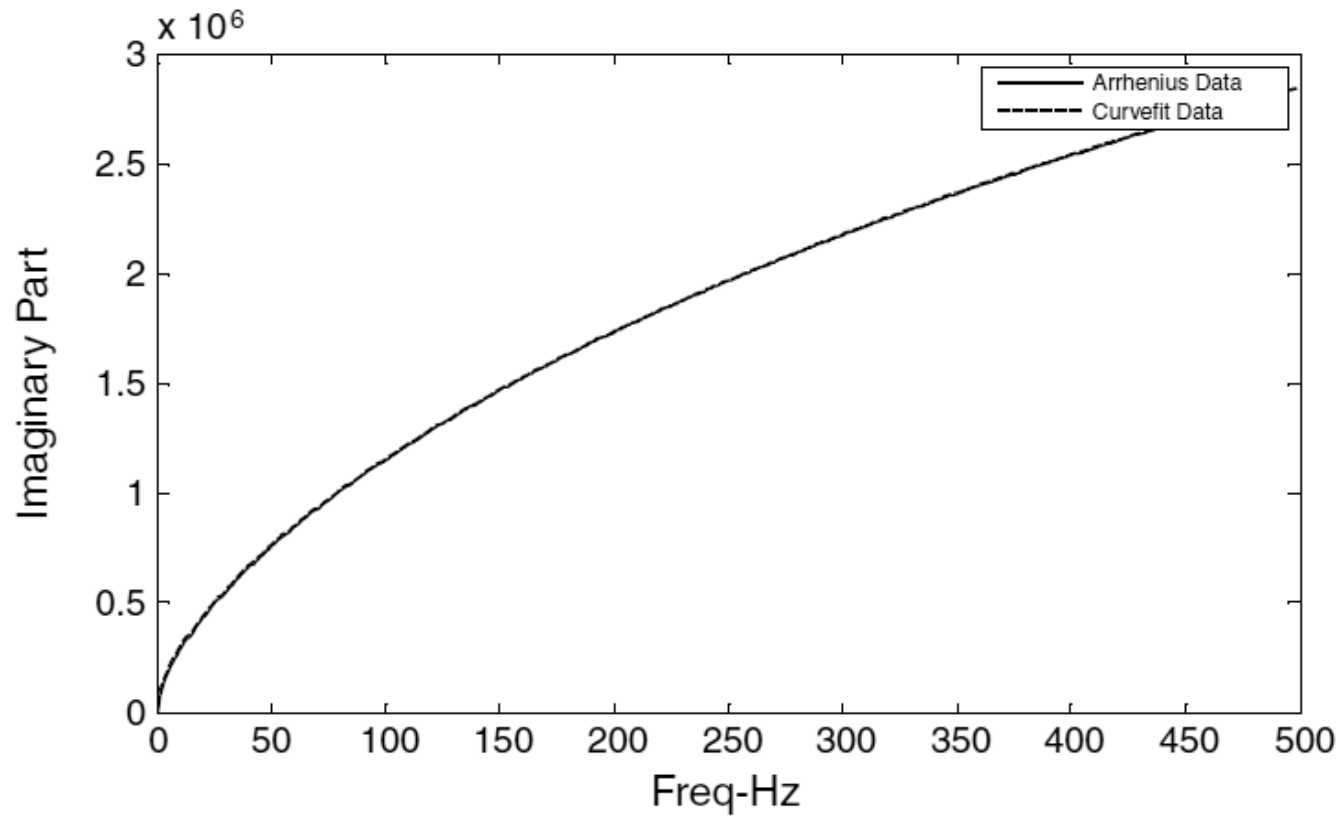

Figure 4.7 Imaginary part comparison of ISD- $11045^{\circ} \mathrm{C}$ 
Chapter 4.5-Parametric Determination of the Biot Damping Model

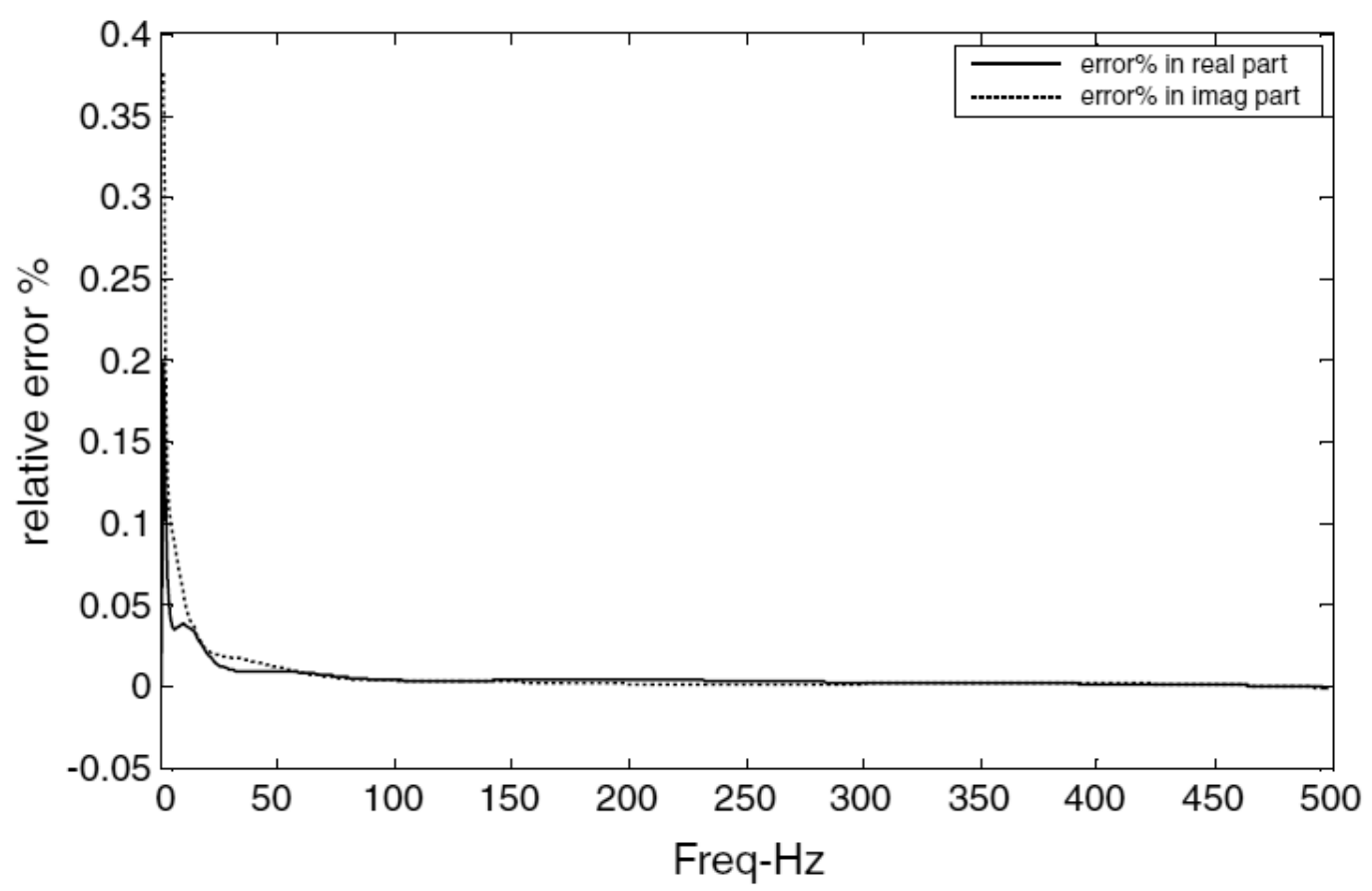

Figure 4.8 Relative error percentage comparison of ISD-110 $45^{\circ} \mathrm{C}$

As shown in the plots above, the dynamic properties of $3 \mathrm{M}$ ISD-110 at $45^{\circ} \mathrm{C}$ can be represented using the Biot parametric determination technique discussed in this section. The relative error between the two sets of data is low; and the higher-frequency region contains almost zero error. 


\section{Acoustical BEM Analysis}

\subsection{Introduction of Acoustical BEM Theory}

In the previous section, the vibration problem of the multiple-layer sandwich beam is solved through the time-domain dynamic ordinary differential equation (ODE) of the Biot damping model with the numerical modeling by FE technique. The vibration problem can be extended to an acoustical problem by the semi-coupled method: the vibration will induce a change in sound pressure, but the sound pressure will not create the vibration.

The boundary element method (BEM) is the most commonly used method in acoustical analysis. The early application of BEM is by Schweikert and Chen [95] in predicting the sound radiation from arbitrary structure. Between 1960s and 1980s, many researchers [96-101] made tremendous contributions to the development of acoustical BEM analysis.

Nowadays, the direct and indirect boundary element methods are available for 
acoustical analysis. The traditional direct method solves sound pressure as primary variable in either a bounded interior domain or an unbounded exterior domain at a time. The flexibility of element type features the direct BEM such as continuous isoperimetric and quadratic elements.

The indirect BEM proposed by Hamdi and other researchers [102-104] solves the Helmholtz equation in both interior and exterior domain simultaneously by calculating the jump of sound pressure or particle velocity across the boundary as variables. The indirect method requires more computational resources as well as certain element types, mostly continuous elements.

In this section, the acoustical interior problem will be numerically solved by the $2 \mathrm{D}$ direct BEM technique $[45,46]$ in a bounded fluid domain V as shown in Figure 5.1.

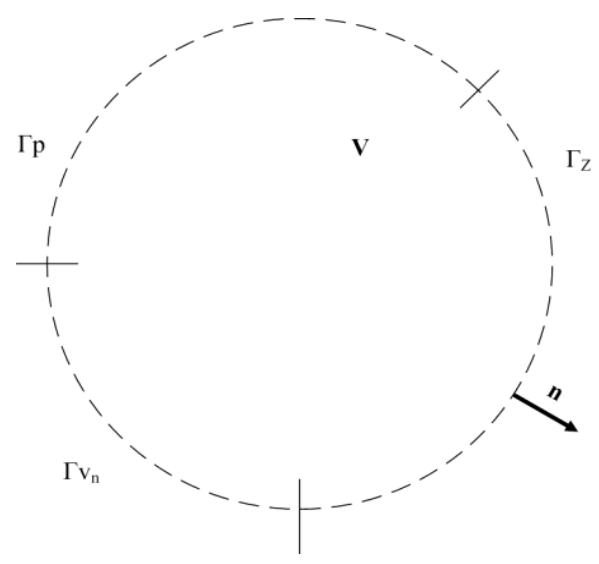

Figure 5.1 Notations of 2D BEM interior problem in fluid domain V 
The sound pressure distribution (p) of the time-harmonic wave in the domain $\mathrm{V}$ satisfies the governing differential equation, well-known as the Helmholtz Equation, associated with the boundary conditions on boundary $\Gamma\left(=\Gamma_{p} \cup \Gamma_{v_{n}} \cup \Gamma_{Z}\right)$ as follows:

$$
\begin{gathered}
\nabla^{2} \mathbf{p}(x)+k^{2} \mathbf{p}(x)=0, \text { when } \\
\mathbf{p}(x)=p_{0}, \quad x \in \Gamma_{p} \\
\mathbf{v}_{\mathbf{n}}(x) \equiv-\frac{1}{j w \rho_{0}} \frac{\partial \mathbf{p}}{\partial n}=\mathbf{v}_{n_{0}}, \quad x \in \Gamma_{v_{n}} \\
\mathbf{z}(x) \equiv \frac{\mathbf{p}}{\mathbf{v}_{\mathbf{n}}}=z_{0}, \quad x \in \Gamma_{Z} .
\end{gathered}
$$

where $\mathrm{k}$ is equal to $\omega / \mathrm{c}$, meaning the wave number is equal to the radiant frequency over the velocity of sound; $\mathrm{v}_{\mathrm{n}}, \rho_{0}, \mathrm{z}$ stand for the normal velocity, density of the fluid V(normally the air), and acoustical impedance of the fluid V respectively.

In this research, the intersection between the vibration and the acoustics problem is the normal velocity on the acoustical boundary. Recalling the dynamic solution of the decoupling transformation, the particle velocity in the time domain of each node can be calculated through Equation (4.54) if the multiple-layer sandwich beam is 
discretized by the FEM; and alternatively the FRF (the complex ratio between the output and input response in the frequency domain) can be determined through the Equation (4.53). Once the input signal is given, the particle velocity or the system displacement versus frequency relationship can be obtained through the FRF.

To solve the governing differential equation (Equation (5.1)) in the bounded fluid domain $\mathrm{V}$, the Helmholtz Equation can be transformed to the integral equation, converting the $2 \mathrm{D}$ area integration to the $1 \mathrm{D}$ curve integration around the area:

$$
c(\xi) p(\xi)+\int_{\Gamma} \frac{\partial \Psi(\xi, x)}{\partial n} p(\xi) d \Gamma=-\int_{\Gamma} i \rho_{0} \omega \Psi(\xi, x) v_{n}(x) d \Gamma
$$

in which:

$c(\xi)=$ geometry-dependent coefficient, normally $c(\xi)=0$ when $\xi$ is in the domain $\mathrm{V}, c(\xi)=0.5$ when $\xi$ is on the smooth boundary $\Gamma$ $p(\xi)=$ sound pressure at source point $\xi$ $\Psi(\xi, x) \mathrm{x}$ is the field point and $\boldsymbol{\Psi}=-\frac{i}{4} \mathbf{H}_{0}^{(2)}(k r)$ for the 2D BEM problem; $\mathrm{r}$ stands for the Euclidian distance between $\mathrm{x}$ and $\xi$; $\mathrm{k}$ is equal to $\omega$ (radiant frequency) divided by c(sound velocity); $\mathbf{H}_{0}^{(2)}$ is the Hankel function of $2^{\text {nd }}$ kind (see Appendix for details)

$\mathrm{n}=$ normal vector pointing away to the fluid domain $\mathrm{V}$ 
By discretizing the boundary into a series of curvilinear elements through the introduction of the shape functions [46], the integral equation can be calculated numerically by solving the following linear matrix:

$$
\mathbf{H P}=\mathbf{G V}_{\mathrm{N}}
$$

where $\mathbf{H}$ comes from the terms of $c(\xi)$ and $\int_{\Gamma} p(\xi) \frac{\partial \Psi(\xi, x)}{\partial n} d \Gamma, \mathrm{G}$ is derived from $-\int_{\Gamma} i \rho_{0} \omega v_{n}(x) \Psi(\xi, x) d \Gamma$ in Equation (5.2), the vector $\mathbf{P}$ and $\mathbf{V}_{\mathbf{N}}$ includes sound pressure and particle velocity values, both unknowns and known from the boundary condition.

Thus, each set of node velocity due to the force input results in one set of solution on the sound pressure by BEM discussed in this section. In summary, through the proposed acoustical BEM, it is possible to figure out the time-harmonic sound pressure distribution corresponding to each single frequency point in the frequency spectrum. 


\subsection{Calculation Details of 2D BEM Analysis}

For this particular acoustical BEM interior problem, the boundary of acoustical cavity is discretisized as 18 quadratic equally-spaced boundary elements. The quadratic curvilinear element has three nodes, and the interpolation between each node represents the geometry of each element. The shape functions [46] are:

$$
N_{1}=\frac{1}{2} \xi(\xi-1) ; \quad N_{2}=(\xi+1)(\xi-1) ; \quad N_{3}=\frac{1}{2} \xi(\xi+1) ;
$$

with respect to the element coordinates:

$$
x=\sum_{i=1}^{3} x_{i} N_{i}(\xi) ; \quad y=\sum_{i=1}^{3} y_{i} N_{i}(\xi)
$$

where $\mathrm{x}_{\mathrm{i}}$ and $\mathrm{y}_{\mathrm{i}}$ are the coordinates at each nodal point, and $\xi$ stands for the local coordinate between -1 and 1 on a master element.

The boundary variables $\mathrm{p}$ and $\mathrm{v}_{\mathrm{n}}$ are also discritezed by quadratic element with the same shape functions representing the geometry:

$$
p=\sum_{i=1}^{3} p_{i} N_{i}(\xi) ; \quad v_{n}=\sum_{i=1}^{3} v_{n i} N_{i}(\xi)
$$


When the seven-layered sandwich beam $(\mathrm{L}=1 \mathrm{~m})$ is simply-supported on the bottom of the acoustical cavity, the sound pressure level of the field point $(x=0.5 \mathrm{~m}, \mathrm{y}=0.4 \mathrm{~m})$ is calculated through this proposed method, and the calculation results will be presented in the next section. The following Figure 5.2 demonstrates the detailed layout of this 2D acoustical cavity problem. The anechoic boundary condition is applied on the inside of the acoustical cavity and the thickness of the multiple-layered beam is neglected for the purpose of approximation.

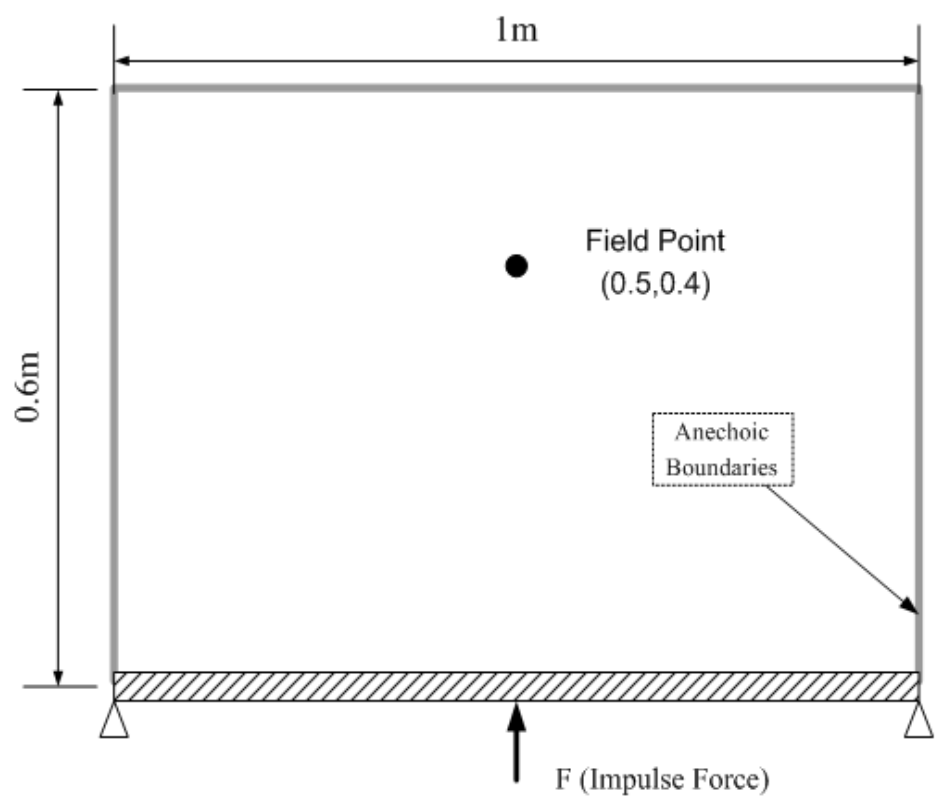

Figure 5.2 Layout of BEM acoustical cavity problem 


\section{Numerical Examples}

\subsection{Example of a 2 DOF Lumped-Mass Model}

A. Case1

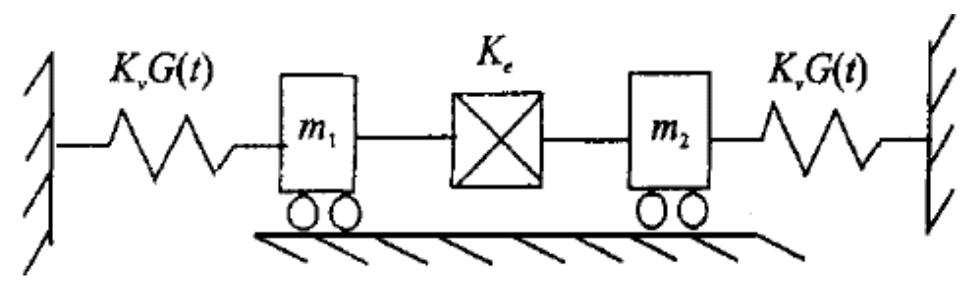

Figure 6.1 Schematic diagram of a lumped mass system- case1

Consider a system with two dampers on both ends as shown above with the relaxation function:

$$
G(t)=4.0+49.8 e^{-1685.6 t}+1196.2 e^{-148314.4 t}
$$

Converting to the Biot damping model form:

$$
s G(s)=4.0 \times\left(1+\frac{12.45 s}{s+1685.6}+\frac{299.05 s}{s+148314.4}\right)
$$


Mass, Elastic stiffness and viscoelastic Stiffness Matrix:

$$
M=\left[\begin{array}{ll}
5 & 0 \\
0 & 1
\end{array}\right], K_{e}=\left[\begin{array}{cc}
10^{5} & -10^{5} \\
-10^{5} & 10^{5}
\end{array}\right] ; K_{v}=\left[\begin{array}{cc}
10^{3} & 0 \\
0 & 10^{3}
\end{array}\right]
$$

Apply the Biot damping model and obtain the complex eigenvalues:

$$
\begin{aligned}
& \ddot{\mathrm{e}}_{1}=-6.1989+35.9488 \mathrm{i} \rightarrow 5.8059 \mathrm{~Hz} / 0.3554 \text { (loss factor) } \\
& \ddot{\mathrm{e}}_{2}=-16.1428+353.5234 \mathrm{i} \rightarrow 56.324 \mathrm{~Hz} / 0.0915 \text { (loss factor) }
\end{aligned}
$$

The time-domain responses for mass $\# 1$ and \#2 are obtained through the decoupling transformation and convolution method discussed in the previous chapter. In this example, the system displacements of the two, mass\#1 and \#2 die out due to the damping with the unit step excitation applied on mass $\# 1$.

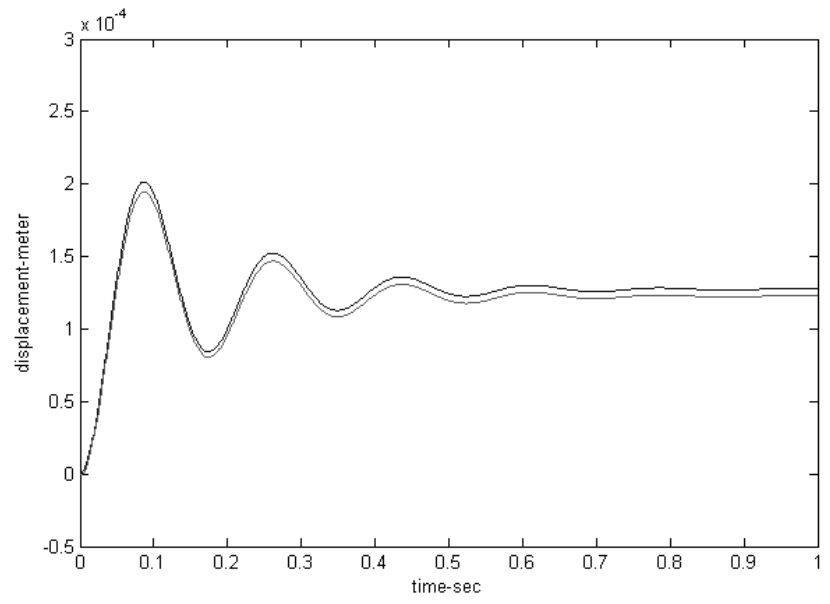

Figure 6.2 System displacements under the unit step excitation on mass\#1 


\section{Case2:}

Another damper between \#1 and \#2 mass is added with another viscoelastic material as shown in Figure 6.3 below

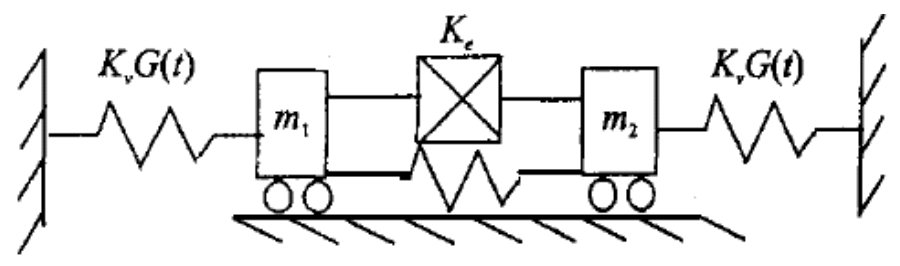

Figure 6.3 Schematic diagram of a lumped-mass system- case 2

The Biot complex shear modulus for the dampers on both ends:

$$
s G(s)=4.0 \times\left(1+\frac{12.45 s}{s+1685.6}+\frac{299.05 s}{s+148314.4}\right)
$$

The Biot complex shear modulus for the damper in the middle:

$$
s G(s)=4.6075 \times\left(1+\frac{1.5122 s}{s+267.3249}+\frac{6.3225 s}{s+2936.3178}+\frac{690.4479 s}{s+371461.7163}\right)
$$

Mass, elastic stiffness and viscoelastic stiffness matrix:

$$
M=\left[\begin{array}{ll}
5 & 0 \\
0 & 1
\end{array}\right], K_{e}=\left[\begin{array}{cc}
10^{5} & -10^{5} \\
-10^{5} & 10^{5}
\end{array}\right] ; K_{v 1}=\left[\begin{array}{cc}
10^{3} & 0 \\
0 & 10^{3}
\end{array}\right] ; K_{v 2}=\left[\begin{array}{cc}
10^{3} & -10^{3} \\
-10^{3} & 10^{3}
\end{array}\right]
$$

Apply the Biot damping model and figure out the complex eigenvalue problem, the following results can be obtained: 


$$
\begin{aligned}
& \ddot{\mathrm{e}}_{1}=-6.2052+35.9527 \mathrm{i} \rightarrow 5.8067 \mathrm{~Hz} \quad / 0.3558 \text { (loss factor) } \\
& \ddot{\mathrm{e}}_{2}=-33.3094+369.4069 \mathrm{i} \rightarrow 59.0315 \mathrm{~Hz} \quad / 0.1818 \text { (loss factor) }
\end{aligned}
$$

Similarly, the system displacements of two mass\#1 and \#2 dissipate out due to the damping with the unit step excitation applied on mass \#1.

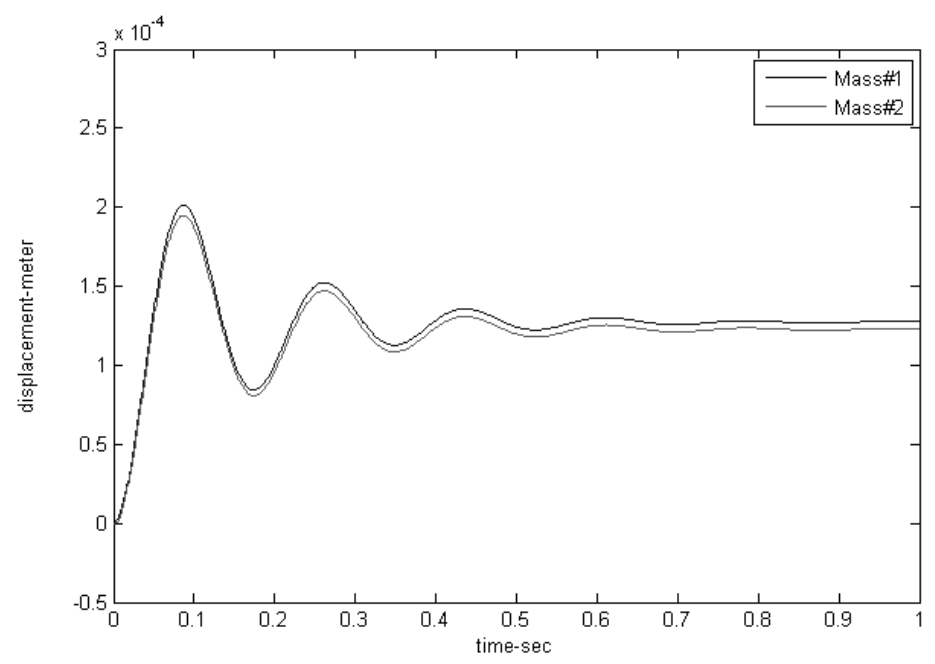

Figure 6.4 System displacements on the unit step excitation on mass\#1

The table below compares computational results from the two scenarios. From the highlighted loss factors, we see that the loss factor almost doubled compared between the lumped-mass systems with and without the damper in the middle, while the frequency of the $2^{\text {nd }}$ mode was slightly changed. Thus the computation result validates the need for this design, which simply involves adding another damper to increase to the system loss factor. 
Table 6.1 Parameters comparison of lumped-mass cases

\begin{tabular}{cccc}
\hline \hline $\begin{array}{c}\text { \# of } \\
\text { Mode }\end{array}$ & & 2DOF & 2DOF \\
\hline & & Ref [53] & 2VEMs \\
\hline & $1^{\text {st }}$ VEM & 2 terms Biot & 2 terms Biot \\
& $2^{\text {nd }}$ VEM & ---- & 3 terms Biot \\
\hline $1^{\text {st }}$ & Frequency & $5.8059 \mathrm{~Hz}$ & $5.8067 \mathrm{~Hz}$ \\
& Loss factor & 0.3554 & 0.3558 \\
$2^{\text {nd }}$ & Frequency & $56.3235 \mathrm{~Hz}$ & $59.0313 \mathrm{~Hz}$ \\
& Loss factor & 0.0915 & 0.1818 \\
\hline \hline
\end{tabular}




\title{
6.2 Example of a Three-Layer Sandwich Beam
}

\author{
A. Damping Material Properties For The Viscoelastic Core
}

In this numerical example, the Biot damping parameters for $3 \mathrm{M}$ ISD- $110\left(45^{\circ} \mathrm{C}\right)$ and ZN1 polymer $[53,54]$ will be used in the simulation. The datasheet below shows the Biot constants curve-fitted from Arrhenius Alpha damping model by Auto2fit, and converted to 6 terms of the Biot Damping parameters:

Table 6.2 Biot parameters of $3 \mathrm{M}$ ISD- $11045^{\circ} \mathrm{C}$

\begin{tabular}{cccc}
\hline \hline$G^{\infty}$ & $55000(\mathrm{~Pa})$ & & \\
$\mathrm{a}_{1}$ & 1.809517 & $\mathrm{~b}_{1}$ & 5.410993 \\
$\mathrm{a}_{2}$ & 14.53095 & $\mathrm{~b}_{2}$ & 1093.778 \\
$\mathrm{a}_{3}$ & 3.221535 & $\mathrm{~b}_{3}$ & 60.36544 \\
$\mathrm{a}_{4}$ & 52.01026 & $\mathrm{~b}_{4}$ & 4319.613 \\
$\mathrm{a}_{5}$ & 19768.22 & $\mathrm{~b}_{5}$ & 2840958 \\
$\mathrm{a}_{6}$ & 6.561162 & $\mathrm{~b}_{6}$ & 298.0672 \\
\hline \hline
\end{tabular}

The fractional derivative damping model of ZN1 damping material in the frequency domain is the raw data for this curve-fitting task:

$$
G(s)=10^{5} \times\left(3.6275+0.2091 s^{0.6752}\right)
$$

Curve-fitting from fractional derivative damping model using Auto2fit, the 4-term 
Biot damping parameters are listed in the datasheet below

\begin{tabular}{|c|c|c|c|}
\hline$G^{\infty}$ & $362750(\mathrm{~Pa})$ & & \\
\hline $\mathrm{a}_{1}$ & 0.762063 & $b_{1}$ & 53.72964 \\
\hline $\mathrm{a}_{2}$ & 1.814626 & $\mathrm{~b}_{2}$ & 504.5871 \\
\hline $\mathrm{a}_{3}$ & 84.93828 & $\mathrm{~b}_{3}$ & 29695.64 \\
\hline $\mathrm{a}_{4}$ & 4.869723 & $\mathrm{~b}_{4}$ & 2478.43 \\
\hline
\end{tabular}

B. Three-Layer damping structure

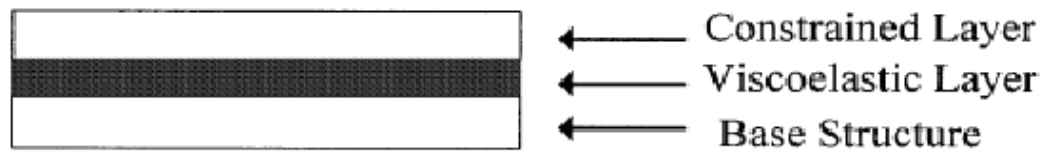

Figure 6.5 Schematic diagram of 3-layer damping structure

A three-layer sandwich beam with viscoelastic cores is shown in Figure 6.5, with the design parameters listed in Table 6.4:

Table 6.4 Design parameters of three-layer structure

\begin{tabular}{|c|c|c|c|}
\hline $\mathrm{L}=1 \mathrm{~m}$ & $\mathrm{t}=0.1 \mathrm{~m}$ & \# of element:12 & \# of nodes: 13 \\
\hline & height of layer & $\begin{array}{c}\text { elastic/shear } \\
\text { properties }\end{array}$ & $\begin{array}{c}\text { material } \\
\text { density }\end{array}$ \\
\hline $1^{\text {st }}$ layer & $\mathrm{h}_{1}=1 \mathrm{~mm}$ & $\mathrm{E}_{1}=210 \mathrm{GPa}$ & $\rho_{1}=7800 \mathrm{~kg} / \mathrm{m}^{3}$ \\
\hline $2^{\text {nd }}$ layer & $\mathrm{h}_{2}=0.8 \mathrm{~mm} ;$ & $\mathrm{G}_{2}$ : Biot & $\rho_{2}=970 \mathrm{~kg} / \mathrm{m}^{3}$ \\
\hline $3^{\text {rd }}$ layer & $\mathrm{h}_{3}=1 \mathrm{~mm}$ & $\mathrm{E}_{3}=210 \mathrm{GPa}$ & $\rho_{3}=7800 \mathrm{~kg} / \mathrm{m}^{3}$ \\
\hline
\end{tabular}


The above data are used to predict the vibration performance of the system using the numerical simulation method presented in this dissertation with the previous publication results [53, 94], as well as simulation results from a commercial software-ANSYS. The different boundary conditions including simply-supported and clamp-free were investigated separately. As far as the curve-fitting result is concerned, $3 \mathrm{M}$ ISD- 110 at $45^{\circ} \mathrm{C}$ is selected for the shear modulus of the viscoelastic layers for the pin-pin boundary condition and ZN1 for cantilever boundary condition. The results are shown in Table 6.5 and 6.6 respectively and the simulation presented in this research shows very good agreement with both previous reference result and numerical result from ANSYS.

Table 6.5 Comparison between the results from simulation and references (pin-pin BC)

\begin{tabular}{cccccc}
\hline \hline $\begin{array}{c}\text { \# of } \\
\text { Mode }\end{array}$ & Pin-pin BC & ANSYS & $\begin{array}{c}\text { Min Hao's } \\
\text { thesis [94] }\end{array}$ & $\begin{array}{c}\text { 8DOF 3-layer } \\
\text { [53] }\end{array}$ & $\begin{array}{c}\text { FE model of } \\
\text { this research }\end{array}$ \\
\cline { 2 - 6 } & Damping & No damping & $\begin{array}{c}\text { ISD110-45C } \\
\text { Arrhenius }\end{array}$ & $\begin{array}{c}\text { ISD110-45C } \\
\text { 6terms Biot }\end{array}$ & $\begin{array}{c}\text { ISD110-45C } \\
\text { 6terms Biot }\end{array}$ \\
\hline $1^{\text {st }}$ & Model & & $3.9454 \mathrm{~Hz}$ & $3.838 \mathrm{~Hz}$ & $3.838 \mathrm{~Hz}$ \\
& Frequency & $3.8498 \mathrm{~Hz}$ & 0.4209 & 0.499 & 0.4992 \\
$2^{\text {nd }}$ & Foss factor & - & $12.4761 \mathrm{~Hz}$ & $12.269 \mathrm{~Hz}$ & $12.269 \mathrm{~Hz}$ \\
& Frequency & $12.501 \mathrm{~Hz}$ & 0.4232 & 0.4461 & 0.4465 \\
$3^{\text {rd }}$ & Loss factor & - & $25.6080 \mathrm{~Hz}$ & $25.302 \mathrm{~Hz}$ & $25.298 \mathrm{~Hz}$ \\
& Frequency & $26.542 \mathrm{~Hz}$ & 0.3746 & 0.3784 & 0.379 \\
$4^{\text {th }}$ & Loss factor & - & $43.3855 \mathrm{~Hz}$ & $42.98104 \mathrm{~Hz}$ & $42.955 \mathrm{~Hz}$ \\
& Frequency & $46.114 \mathrm{~Hz}$ & 0.3305 & 0.321 & 0.3218 \\
$5^{\text {th }}$ & Loss factor & - & $65.94 \mathrm{~Hz}$ & $65.41 \mathrm{~Hz}$ & $65.31 \mathrm{~Hz}$ \\
& Frequency & $71.23 \mathrm{~Hz}$ & 0.2946 & 0.28 & 0.2811 \\
\hline \hline
\end{tabular}


Table 6.6 Comparison between the results from simulation and references (fixed-free BC)

\begin{tabular}{ccccc}
\hline \hline $\begin{array}{c}\text { \# of } \\
\text { Mode }\end{array}$ & $\begin{array}{c}\text { Fixed-free } \\
\text { BC }\end{array}$ & ANSYS & $\begin{array}{c}\text { 8DOF 3-layer } \\
{[53]}\end{array}$ & $\begin{array}{c}\text { FE model of } \\
\text { this research }\end{array}$ \\
\cline { 2 - 5 } & Damping & No damping & $\begin{array}{c}\text { ZN1 4terms } \\
\text { Biot }\end{array}$ & $\begin{array}{c}\text { ZN1 4terms } \\
\text { Biot }\end{array}$ \\
\hline $1^{\text {st }}$ & Fodel & & $1.99723 \mathrm{~Hz}$ & $1.99744 \mathrm{~Hz}$ \\
& Loss factor & - & 0.0973 & 0.0974 \\
$2^{\text {nd }}$ & Frequency & $9.0768 \mathrm{~Hz}$ & $8.83547 \mathrm{~Hz}$ & $8.83691 \mathrm{~Hz}$ \\
& Loss factor & - & 0.2655 & 0.2658 \\
$3^{\text {rd }}$ & Frequency & $21.3345 \mathrm{~Hz}$ & $20.9222 \mathrm{~Hz}$ & $20.9258 \mathrm{~Hz}$ \\
& Loss factor & - & 0.3231 & 0.3236 \\
$4^{\text {th }}$ & Frequency & $38.317 \mathrm{~Hz}$ & $36.5548 \mathrm{~Hz}$ & $36.559 \mathrm{~Hz}$ \\
& Loss factor & - & 0.3423 & 0.343 \\
$5^{\text {th }}$ & Frequency & $60.817 \mathrm{~Hz}$ & $57.1488 \mathrm{~Hz}$ & $57.1521 \mathrm{~Hz}$ \\
& Loss factor & - & 0.3346 & 0.3355 \\
\hline \hline
\end{tabular}




\subsection{Vibro-Acoustic Response Example of a Seven-Layer Beam}

A. Comparison with published data

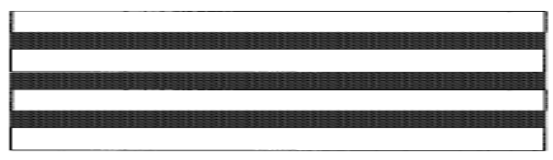

Figure 6.6 Seven-layer sandwich structure with viscoelastic cores

In Figure 6.6, a seven-layer sandwich beam with viscoelastic cores is shown with

the following design parameters listed in Table 6.7:

Table 6.7 Design parameters of seven-layer structure

\begin{tabular}{cccc}
\hline \hline Length: $1 \mathrm{~m}$ & $\begin{array}{c}\text { Thickness: } \\
0.1 \mathrm{~m}\end{array}$ & \# of element:12 & \# of nodes:13 \\
& height of layer & $\begin{array}{c}\text { elastic/viscoelastic } \\
\text { properties }\end{array}$ & $\begin{array}{c}\text { material } \\
\text { density }\end{array}$ \\
\hline \# of layer & $\mathrm{h}_{1}=1 \mathrm{~mm} ;$ & $\mathrm{E}_{1}=210 \mathrm{GPa}$ & $\rho_{1}=7800 \mathrm{~kg} / \mathrm{m}^{3}$ \\
& $\mathrm{~h}_{2}=0.8 \mathrm{~mm} ;$ & $\mathrm{G}_{2}:$ Biot & $\rho_{2}=970 \mathrm{~kg} / \mathrm{m}^{3}$ \\
$1^{\text {st }}$ & $\mathrm{h}_{3}=1 \mathrm{~mm} ;$ & $\mathrm{E}_{3}=210 \mathrm{GPa}$ & $\rho_{3}=7800 \mathrm{~kg} / \mathrm{m}^{3}$ \\
$2^{\text {nd }}$ & $\mathrm{h}_{4}=0.8 \mathrm{~mm} ;$ & $\mathrm{G}_{4}:$ Biot & $\rho_{4}=970 \mathrm{~kg} / \mathrm{m}^{3}$ \\
$3^{\text {rd }}$ & $\mathrm{h}_{5}=1 \mathrm{~mm} ;$ & $\mathrm{E}_{5}=210 \mathrm{GPa}$ & $\rho_{5}=7800 \mathrm{~kg} / \mathrm{m}^{3}$ \\
$4^{\text {th }}$ & $\mathrm{h}_{6}=0.8 \mathrm{~mm} ;$ & $\mathrm{G}_{6}:$ Biot & $\rho_{6}=970 \mathrm{~kg} / \mathrm{m}^{3}$ \\
$5^{\text {th }}$ & $\mathrm{h}_{7}=1 \mathrm{~mm} ;$ & $\mathrm{E}_{7}=210 \mathrm{GPa}$ & $\rho_{7}=7800 \mathrm{~kg} / \mathrm{m}^{3}$ \\
$6^{\text {th }}$ & & & \\
$7^{\text {th }}$ & & &
\end{tabular}

The above data are used to predict the vibration performance of the system using the numerical simulation method presented in this paper with the closed-form solution of Hao and Rao [20,94] as well as simulation results from a commercial 
software-ANSYS. In the ANSYS simulation, the 2D element of Plane 182 (4 Node) is selected and the material damping effect is neglected. Thus, it can be expected that the natural frequency of each mode from ANSYS results will be consistently higher than the result considering the damping effect. As far as the curve-fitting result is concerned, $3 \mathrm{M}$ ISD- 110 at $45^{\circ} \mathrm{C}$ in section IV is selected for the shear modulus of the viscoelastic layers in this numerical example. The different boundary conditions including simply-supported and clamp-free were investigated separately. The results are shown in Table $3 \mathrm{a}$ and $3 \mathrm{~b}$ respectively. It is seen that the agreement between the simulation presented in this paper, the closed-form solution and numerical result from ANSYS is very good for the simply-supported case. When the fixed-free boundary condition is applied, the simulation result presented in this paper follows a trend identical to the ANSYS simulation result. This validates the analysis methodology proposed in the paper. 
Chapter 6.3-Vibro-Acoustic Response Example of a Seven-Layer Beam

Table 6.8 Comparison of results for simply supported boundary condition

\begin{tabular}{ccccc}
\hline \hline $\begin{array}{c}\text { \#of } \\
\text { Mode }\end{array}$ & $\begin{array}{c}\text { Damping } \\
\text { Model }\end{array}$ & No damping & Hao [94] & $\begin{array}{c}\text { FE model of } \\
\text { ISD110-45 }{ }^{\circ} \mathrm{C} \\
\text { this research } \\
\text { ISD } 110-45^{\circ} \mathrm{C}\end{array}$ \\
& & & $\begin{array}{c}\text { Arrhenius } \\
6 \text { terms Biot }\end{array}$ & \\
\hline $1^{\text {st }}$ & Frequency & $6.037 \mathrm{~Hz}$ & $4.7443 \mathrm{~Hz}$ & $4.5834 \mathrm{~Hz}$ \\
& Loss factor & & 0.6248 & 0.7916 \\
$2^{\text {nd }}$ & Frequency & $15.764 \mathrm{~Hz}$ & $13.902 \mathrm{~Hz}$ & $13.9489 \mathrm{~Hz}$ \\
& Loss factor & & 0.6008 & 0.6824 \\
$3^{\text {rd }}$ & Frequency & $30.103 \mathrm{~Hz}$ & $27.661 \mathrm{~Hz}$ & $27.7721 \mathrm{~Hz}$ \\
& Loss factor & & 0.5317 & 0.5632 \\
$4^{\text {th }}$ & Frequency & $49.611 \mathrm{~Hz}$ & $46.1548 \mathrm{~Hz}$ & $47.1053 \mathrm{~Hz}$ \\
& Loss factor & & 0.4715 & 0.4681 \\
$5^{\text {th }}$ & Frequency & \multirow{2}{*}{$74.434 \mathrm{~Hz}$} & $69.3118 \mathrm{~Hz}$ & $68.82259 \mathrm{~Hz}$ \\
& Loss factor & & 0.421 & 0.4226 \\
\hline \hline
\end{tabular}

Table 6.9 Comparison of results for fixed-free boundary condition

\begin{tabular}{|c|c|c|c|}
\hline $\begin{array}{c}\text { \#of } \\
\text { Mode }\end{array}$ & $\begin{array}{l}\text { Damping } \\
\text { Model }\end{array}$ & $\begin{array}{c}\text { ANSYS } \\
\text { No damping }\end{array}$ & $\begin{array}{l}\text { FE model of } \\
\text { this research } \\
\text { ZN1 } 4 \text { terms } \\
\text { Biot [53] }\end{array}$ \\
\hline $1 \mathrm{st}$ & $\begin{array}{l}\text { Frequency } \\
\text { Loss factor }\end{array}$ & $3.5343 \mathrm{~Hz}$ & $\begin{array}{c}2.8453 \mathrm{~Hz} \\
0.2351\end{array}$ \\
\hline $2^{\text {nd }}$ & $\begin{array}{l}\text { Frequency } \\
\text { Loss factor }\end{array}$ & $11.885 \mathrm{~Hz}$ & $\begin{array}{c}11.35425 \mathrm{~Hz} \\
0.4011\end{array}$ \\
\hline $3^{\text {rd }}$ & $\begin{array}{l}\text { Frequency } \\
\text { Loss factor }\end{array}$ & $25.604 \mathrm{~Hz}$ & $\begin{array}{c}24.2774 \mathrm{~Hz} \\
0.4969\end{array}$ \\
\hline $4^{\text {th }}$ & $\begin{array}{l}\text { Frequency } \\
\text { Loss factor }\end{array}$ & $44.635 \mathrm{~Hz}$ & $\begin{array}{c}40.756 \mathrm{~Hz} \\
0.5268\end{array}$ \\
\hline $5^{\text {th }}$ & $\begin{array}{l}\text { Frequency } \\
\text { Loss factor }\end{array}$ & $68.813 \mathrm{~Hz}$ & $\begin{array}{c}62.4374 \mathrm{~Hz} \\
0.5172\end{array}$ \\
\hline
\end{tabular}


B. Vibration performance of a combination of several viscoelastic materials at different temperatures

An important factor affecting the dynamic properties of a system with viscoelastic damping is the temperature. With an increase in temperature, the loss factor approaches its best performance towards the transition region and then decreases afterwards. In this example, the 3M-ISD110 has better damping performance than ISD112 over the chosen temperature between 40 and 60 degree Celsius. It is of interest to study the effect of the combination of these two viscoelastic materials on the damping of the structure.

To introduce the different viscoelastic materials, a seven-layer sandwich beam with the same parameters as in the previous example, incorporating two damping materials (3M ISD110 and ISD112), is designed. This system is compared to the same structure with only one damping material (either 3M ISD110 or ISD112). In the system including two viscoelastic materials, the outer damping layers $\left(2^{\text {nd }}\right.$ and $\left.6^{\text {th }}\right)$ are $3 \mathrm{M}$ ISD112 and the inner damping layer $\left(5^{\text {th }}\right)$ is the $3 \mathrm{M}$ ISD110. Two different scenarios of boundary conditions are examined in this numerical example and the temperature range is from 40 to 60 Degree Celsius. The Figure $6.7 \mathrm{a}$ and $6.7 \mathrm{~b}$ show 
the $1^{\text {st }}$ order natural frequency and the system loss factor over the temperature range

with the simply-supported boundary condition applied to the FE model.
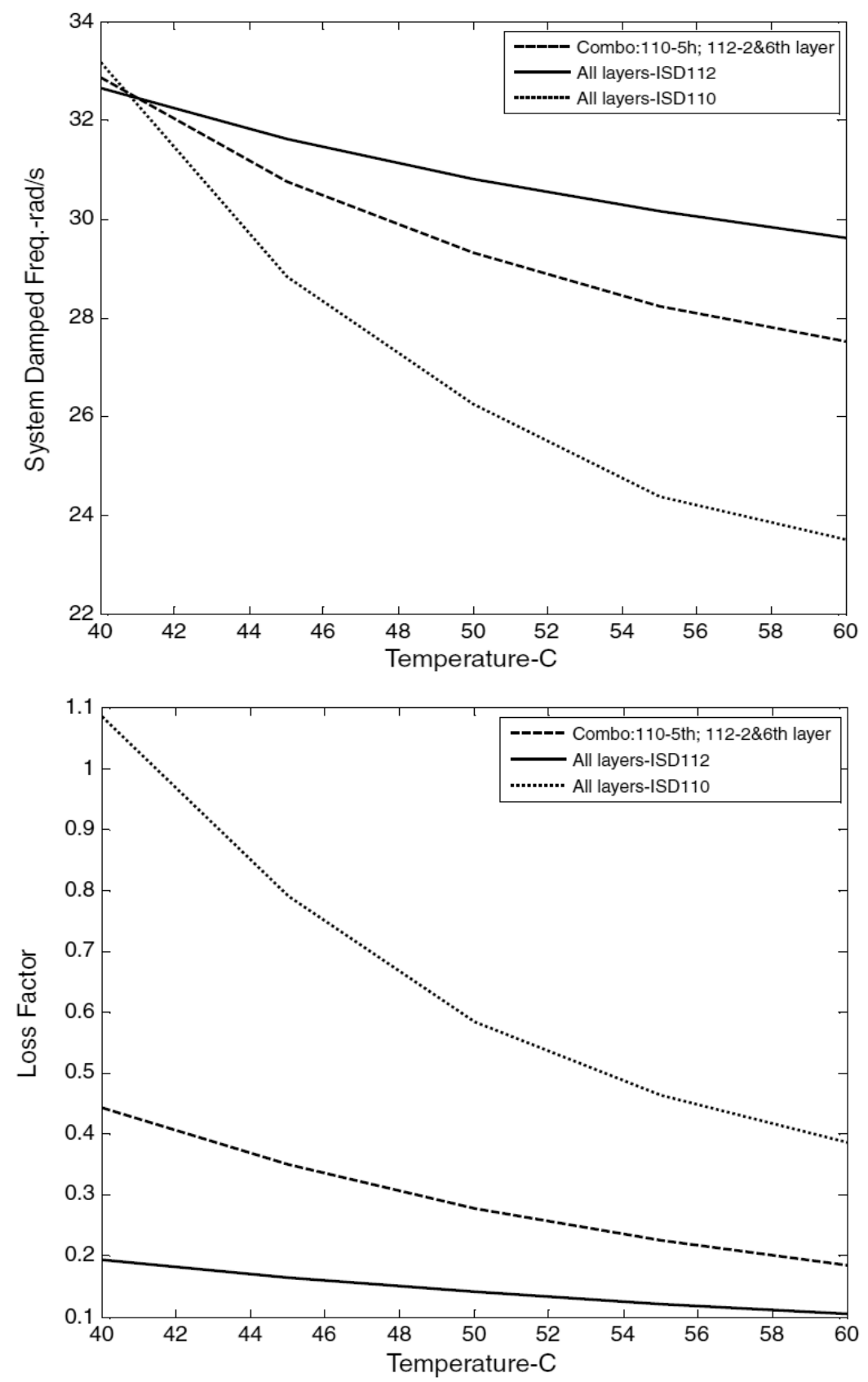

Figure 6.7a-b $1^{\text {st }}$ order system damped frequency/loss factor (simply-supported B.C.) 
On the other hand, the Figure $6.8 \mathrm{a}$ and $6.8 \mathrm{~b}$ show the $1^{\text {st }}$ order vibration performance including natural frequency and loss factor for the fixed-free boundary condition applied on this seven-layer structure. Considering the loss factor of the first flexible mode of the simply-supported system, the same structure with fixed-free boundary condition has less system damping at the corresponding temperature. It can be explained that the simply-supported boundary condition definitely has the advantage when it comes to maximum usage of the shear strain energy in the viscoelastic damping layer.

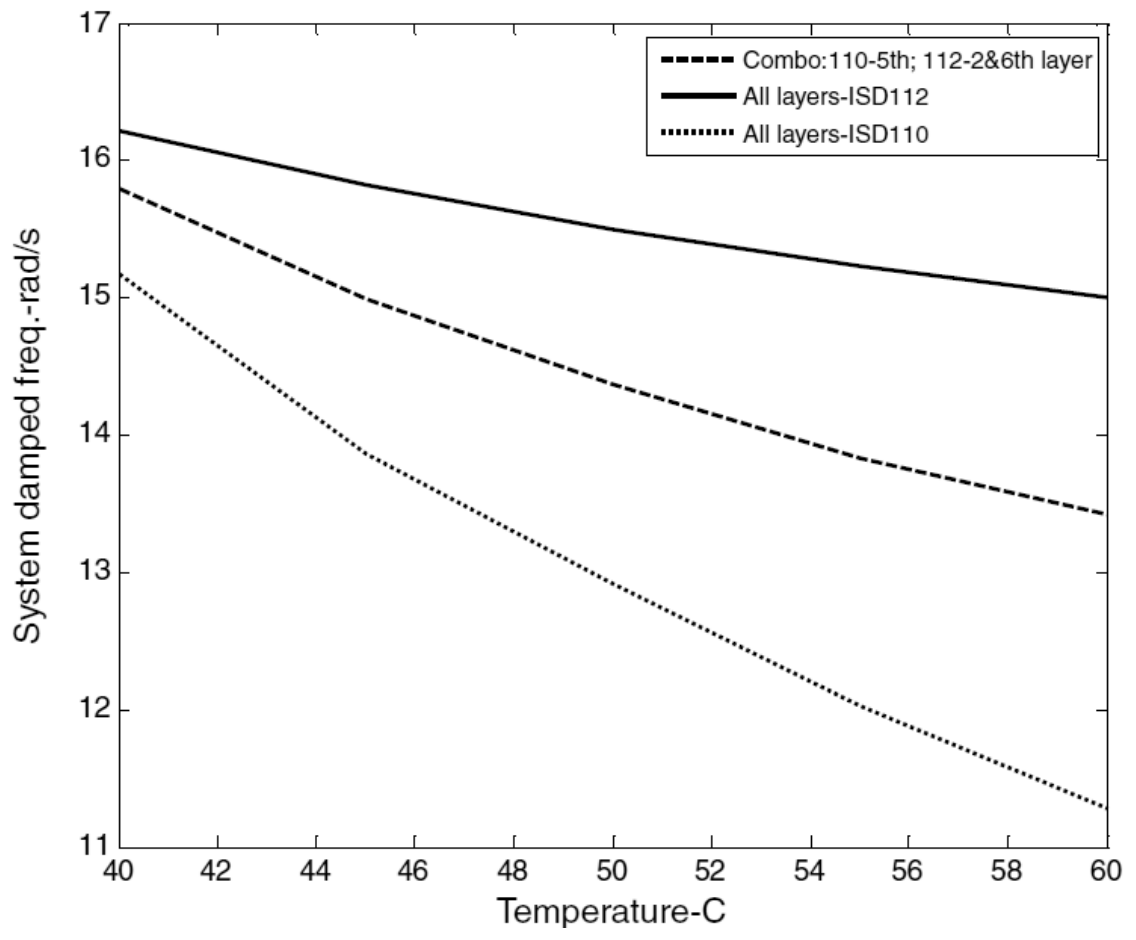




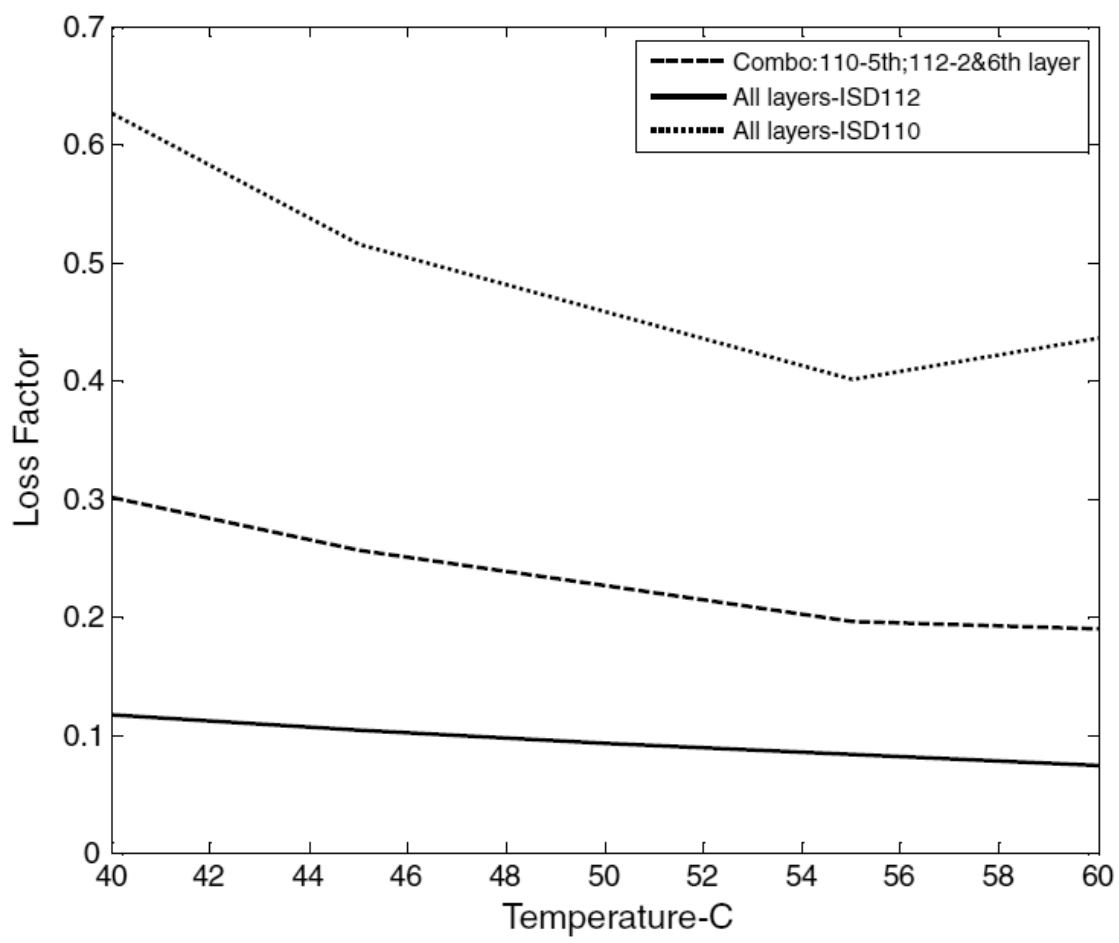

Figure 6.8a-b First damped frequency and loss factor

(Fixed-Free B.C.)

C. Time-domain response under the arbitrary input

The following plot shows the transverse displacement of two nodes(\#2 and \#7 node)

with a unit input in time domain vertically applied on the middle (\#7 node) of the simply-supported seven-layer sandwich beam with the same design parameters as the previous example. The curve-fitting result of 3M ISD110 at the ambient temperature of $45^{\circ}$ Celsius is used in this example. This explains the energy dissipation phenomena in the time domain when an arbitrary force is applied on the structure. 
Chapter 6.3-Vibro-Acoustic Response Example of a Seven-Layer Beam

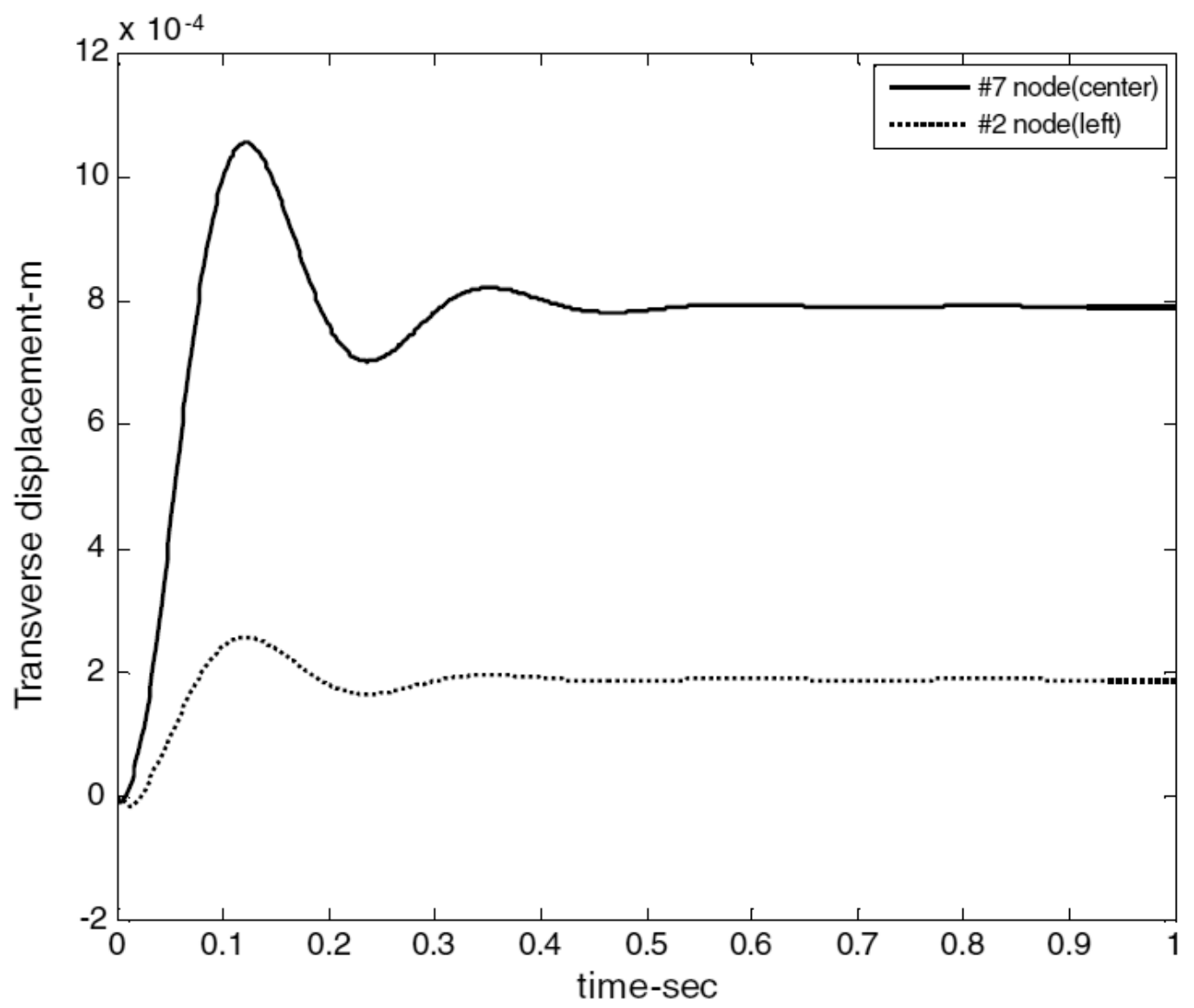

Figure 6.9 System displacements under the unit step excitation 
D. Frequency-spectrum analysis under the arbitrary input

The Figure 6.10 shows the transverse velocity of the middle node (\#7) with a $10 \mathrm{~N}$ step input (frequency domain) vertically applied on the middle (\#7) of the simply-supported seven-layer sandwich beam with the same design parameters as the previous example. The curve-fitting result of 3M ISD110 at the ambient temperature of $45^{\circ} \mathrm{C}$ is used in this example. This pivotal result is the key to extending the vibration problem to an acoustical problem in the frequency domain when an arbitrary force is applied on the structure.

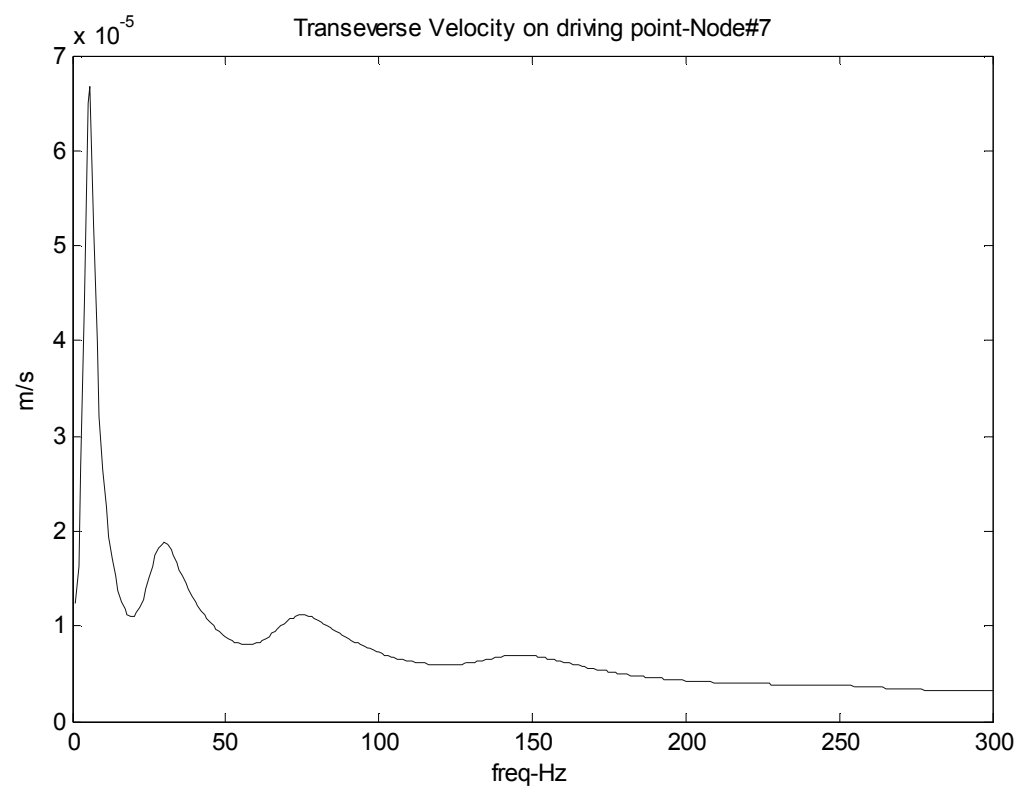

Figure 6.10 Transverse velocity under the impulse excitation (at middle node \#7) 


\section{E. 2D Acoustical BEM results}

The Figure 6.11 illustrates the contour plot $(\mathrm{f}=10 \mathrm{~Hz})$ of sound pressure level (SPL)

when the seven-layer sandwich beam (using $3 \mathrm{M}$ ISD-110 at $45^{\circ} \mathrm{C}$; same design parameters as previous vibration analysis) is subjected to a $10 \mathrm{~N}$ force step input (frequency domain) at the middle node.

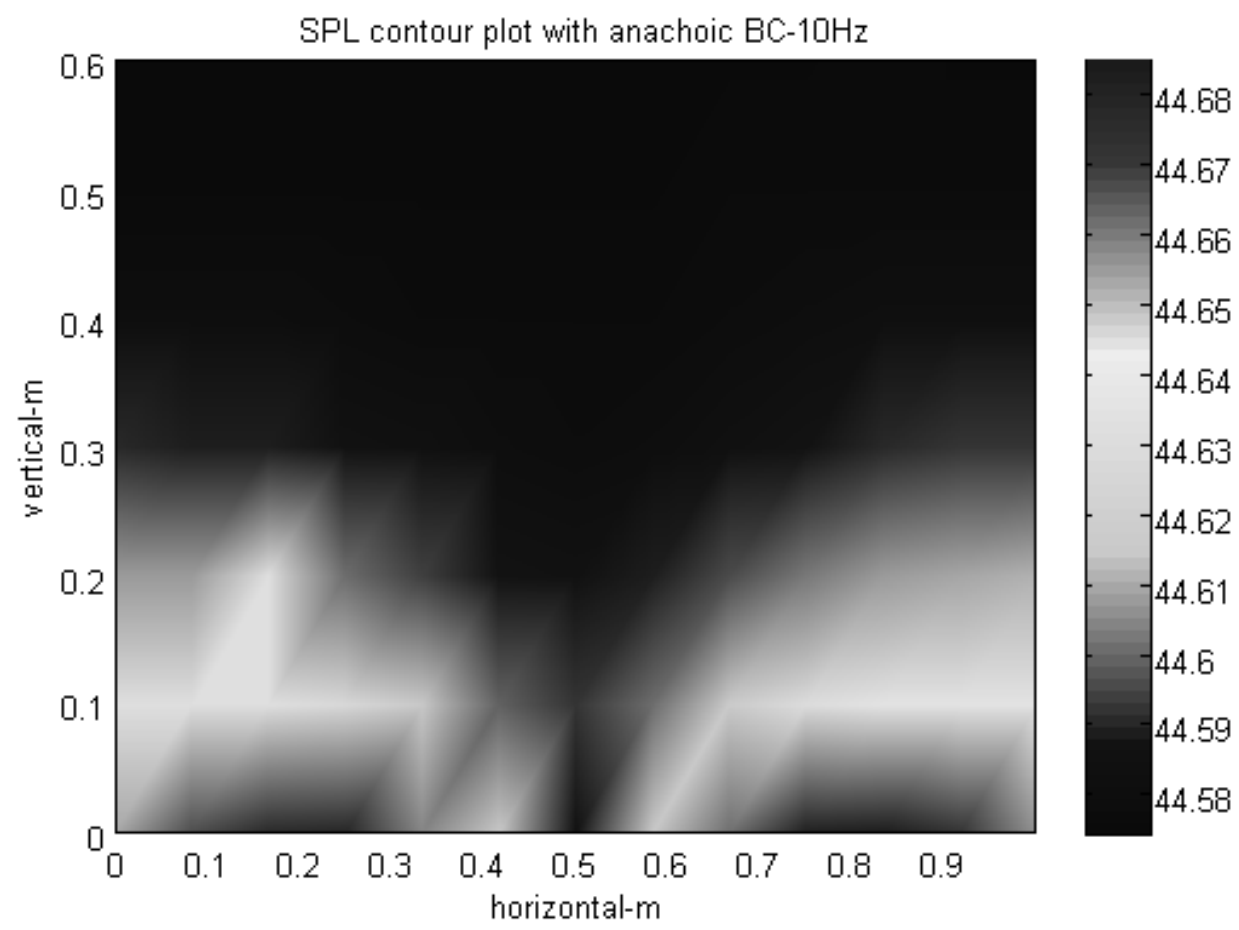

Figure 6.11 Contour plot of SPL when the impulse force applied

$(10 \mathrm{~Hz})$ 
Figure 6.12a shows the continuous sound pressure distribution in the acoustical cavity with the anechoic boundary condition. Figure $6.12 \mathrm{~b}$ extracts the frequency spectrum of Sound Pressure Level (SPL) on the field point $(0.5,0.4 \mathrm{~m})$ indicated by a dot in Figure 6.12. From the result of Figure 6.13, it can be found that the dominant contribution is due to the peak value of the first flexible vibration mode, which agrees with the frequency-spectrum analysis of the vibration problem.

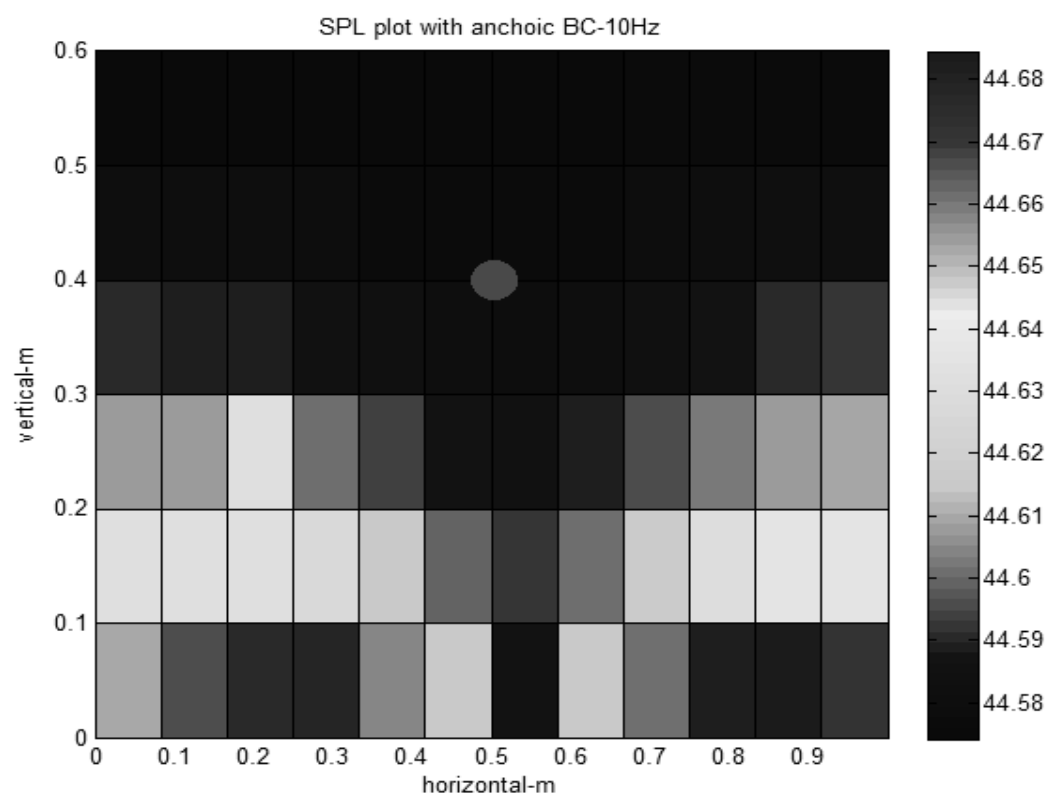

Figure 6.12 Element result of sound pressure level $(10 \mathrm{~Hz})$ 


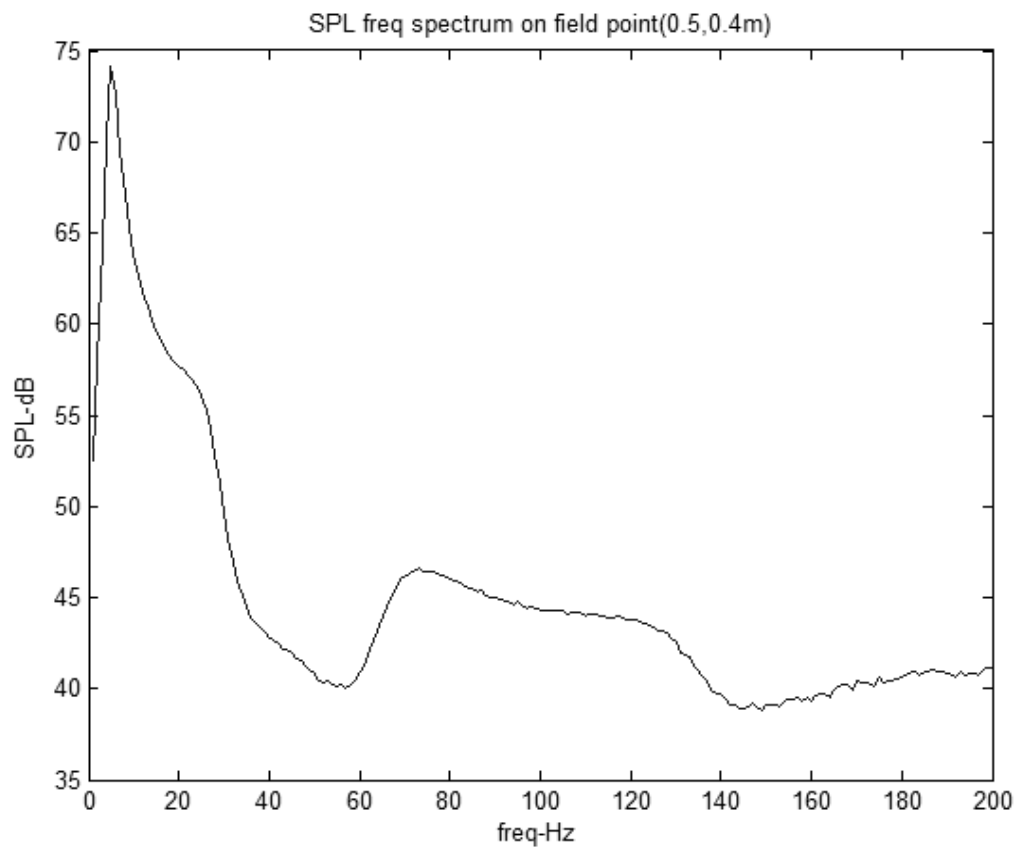

Figure 6.13 Nodal frequency spectrum on $(0.5,0.4 \mathrm{~m})$

F. Comparison with vibro-acoustical software results:

In this section, the hybrid FE-BE model of sandwich damping beam without the viscoelastic damping properties is being developed in commercial software package-ANSYS.ADPL and LMS Virtual. Lab- Acoustics. The harmonic vibration analysis is conducted in ANSYS.APDL module and the frequency-spectrum of field point SPL is calculated in Virtual.lab Acoustics module for the comparison with the SPL frequency spectrum of the viscoelastic damping beam in last section. The 
complete analysis sequence consists of the following steps:

1. Building the FE model and applying the boundary conditions in ANSYS ADPL. The 8-node element SOLID45 (element size $=10 \mathrm{~mm}$ for each layer) is used to build the 3D seven-layer model. The design parameters are identical with the parameters in Table 6.7 for the comparison and the geometry boundary condition is simply-supported. A $10 \mathrm{~N}$ force on each frequency point is applied at the middle nodes. The FE model of 3D sandwich beam is shown in Figure 6.14.

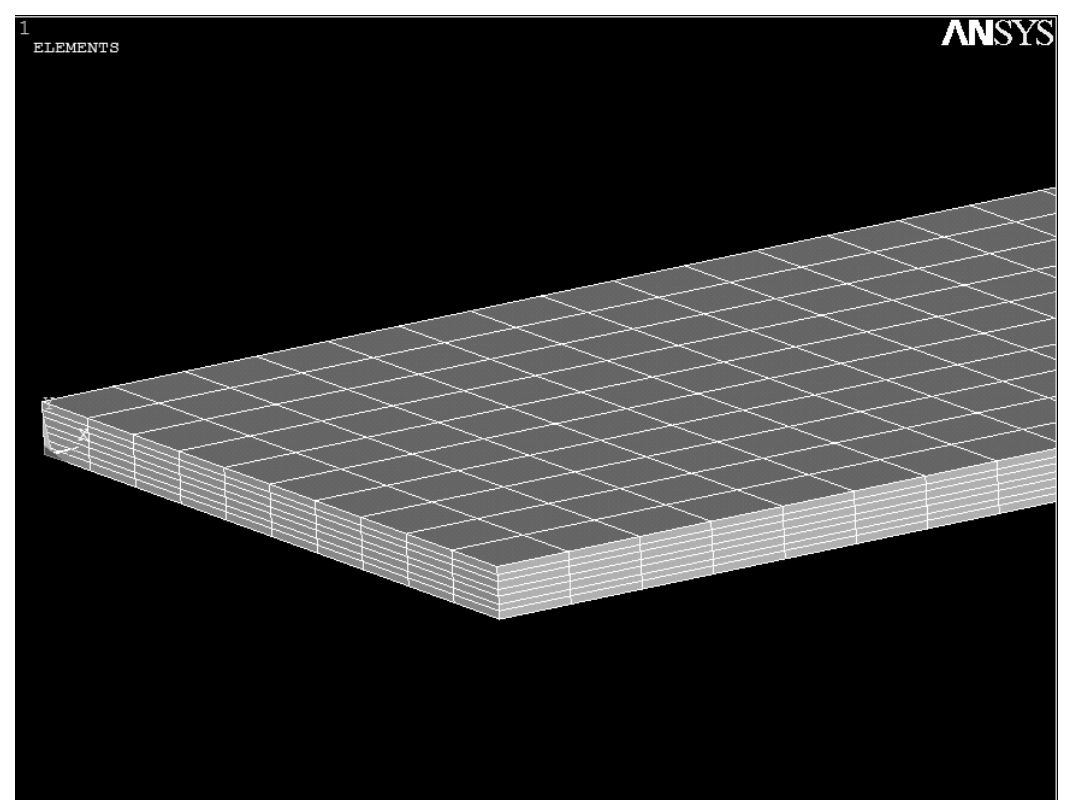

Figure 6.14 Element layout of 3D seven-layer damping beam

2. Conducting the harmonic vibration analysis in ANSYS ADPL. The harmonic 
analysis is used to calculate the nodal displacements for a forced vibration problem in the frequency domain. The frequency range is $0-200 \mathrm{~Hz}$ as $2 \mathrm{~Hz}$ for each step. The full method is being utilized in this analysis and the difference of full-method versus modal-superposition method can be referred to [105]. The comparison of system frequencies between ANSYS modal results and calculation result by Biot dynamic equation is shown in Table 6.10. It implies that the 3D model built in ANSYS.APDL has good correlation with the FE model with the viscoelastic material properties.

Table 6.10 Comparison of modal results with ANSYS simulations

\begin{tabular}{ccccc}
\hline \hline $\begin{array}{c}\text { \#of } \\
\text { Mode }\end{array}$ & $\begin{array}{c}\text { Damping } \\
\text { Model }\end{array}$ & No damping & No damping & $\begin{array}{c}\text { ANS model of } \\
\text { ISD 110-45 } \\
\text { this } \\
6 \text { terms Biot }\end{array}$ \\
\hline $1^{\text {st }}$ & Frequency & $6.037 \mathrm{~Hz}$ & $6.4803 \mathrm{~Hz}$ & $4.5834 \mathrm{~Hz}$ \\
& Loss factor & & & 0.7916 \\
$2^{\text {nd }}$ & Frequency & $15.764 \mathrm{~Hz}$ & $14.338 \mathrm{~Hz}$ & $13.9489 \mathrm{~Hz}$ \\
& Loss factor & & & 0.6824 \\
$3^{\text {rd }}$ & Frequency & $30.103 \mathrm{~Hz}$ & $27.989 \mathrm{~Hz}$ & $27.7721 \mathrm{~Hz}$ \\
& Loss factor & & & 0.5632 \\
\hline \hline
\end{tabular}

3. The BEM mesh is prepared in LMS Virtual. Lab Pre-Acoustics Module. It converts from a solid FE model to a skin mesh that BEM analysis requires. 
The BEM mesh can be seen as a wrap around the structural mesh and usually the BEM mesh is coarser.

4. Calculating the sound pressure in LMS Virtual.lab Acoustics module. Both acoustical and structural meshes are imported to VL.Acoustics. The nodal displacement on each vibration mode calculated in ANSYS.APDL is also imported and mesh-mapped to the acoustical skin mesh as the vibration boundary condition. The location of field plane and field point is consistent with the 2D BEM analysis in this research and the field plane and BEM meshes are shown in Figure 6.15. The acoustical pressure is solved over the frequency range from 2 to $200 \mathrm{~Hz}$. The detail procedure of the analysis sequence can be referred to the training material [106]. 


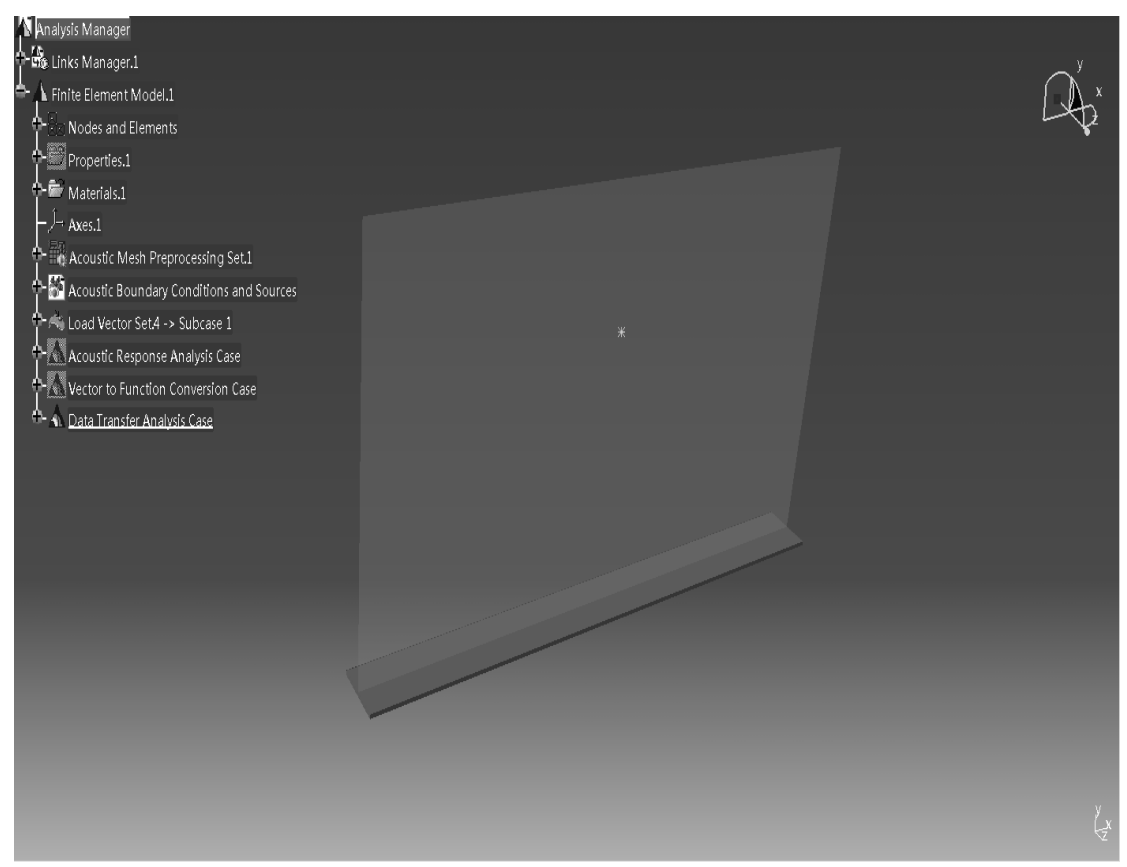

Figure 6.15 Acoustical BEM analysis in LMS Virtual.Lab

As shown in Figure 6.16, 2D BEM calculation has good agreement with the first dominant SPL peak with VL.Acoustic result without considering the damping. Comparing to the structure without damping, the introduction of viscoelastic damping material not only causes almost $20 \mathrm{~dB}$ reduction on the first peak SPL, but also attenuates the other peak values. It proves that using the viscoelastic damping material will greatly attenuate the vibro-acoustical responses comparing to the identical structure without damping. 
Chapter 6.3-Vibro-Acoustic Response Example of a Seven-Layer Beam

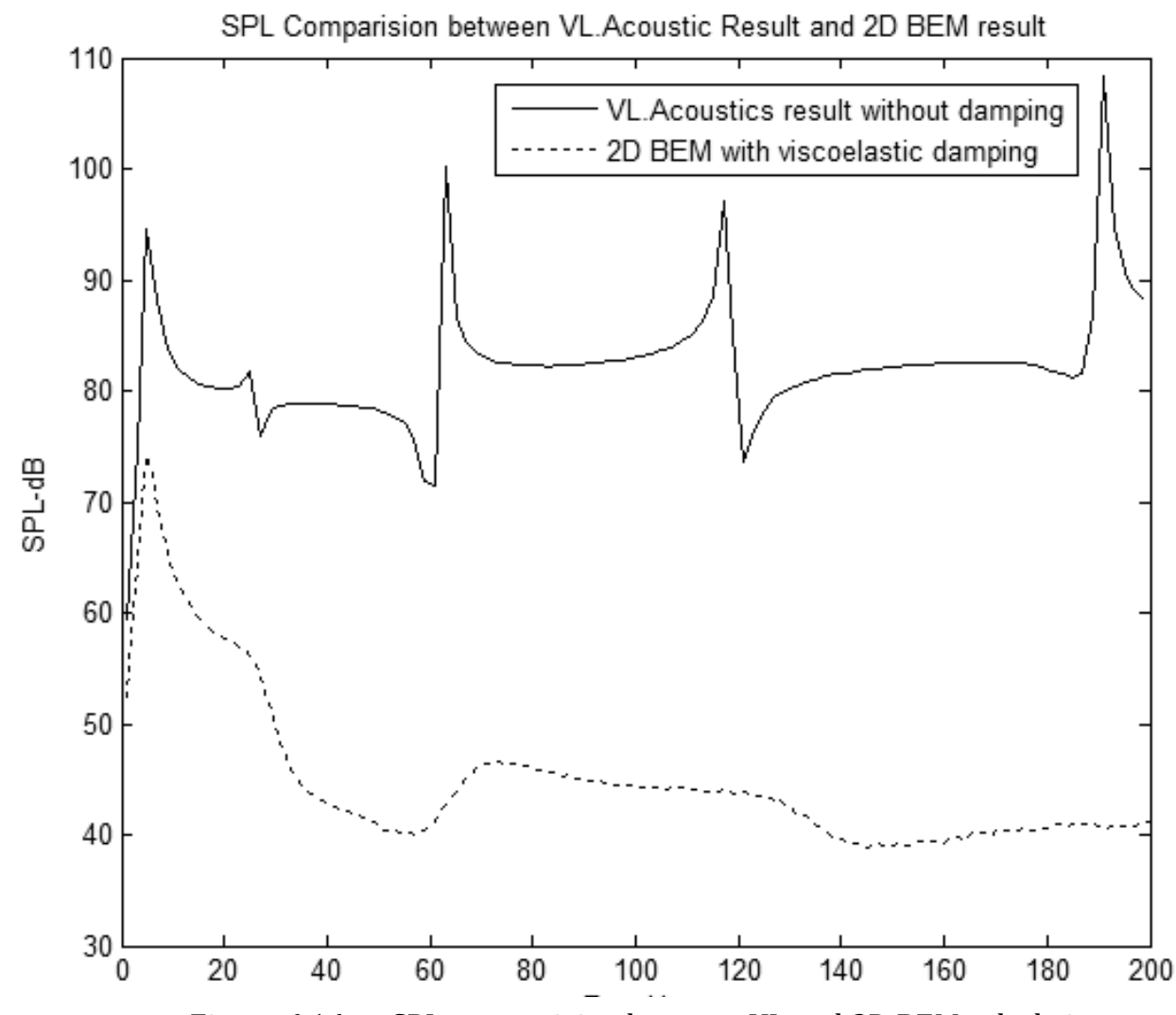

Figure 6.16 SPL comparision between VL and 2D BEM calculation at field point $(0.5,0.4)$ 
G. Acoustical performance with a combination of several viscoelastic materials at different temperatures

With the different combination of viscoelastic materials by changing the ambient temperature, the acoustical response is also calculated with the unit-input in the frequency domain (equivalent to Dirac input [107] in the time domain) applying at the middle node. The damping structure is with the same design parameters as previous vibration analysis (using $3 \mathrm{M}$ ISD-110 at $45^{\circ} \mathrm{C}$ ). The Table 6.11 shows the 1 st order natural frequency, the system loss factor, and the corresponding peak value (dB) of the sound pressure level over the temperature range with the same structure in previous vibration analysis. 
Table 6.11 1 st system damped frequency/loss factor/SPL (simply-supported B.C.)

\begin{tabular}{ccccc}
\hline \hline Type & Temp ${ }^{\circ} \mathrm{C}$ & Freq-rad/s & Loss-factor & SPL-dB \\
\hline ISD-110 & 40 & 33.17 & 1.0850 & 50.35 \\
& 45 & 28.82 & 0.7899 & 51.91 \\
& 50 & 26.25 & 0.5847 & 54.15 \\
& 55 & 24.38 & 0.4640 & 56.24 \\
& 60 & 23.50 & 0.3870 & 57.45 \\
\hline ISD-112 & Temp $^{\circ}{ }^{\circ} \mathrm{C}$ & Freq-rad/s & Loss-factor & SPL-dB \\
\hline & 40 & 32.65 & 0.1946 & 59.54 \\
& 45 & 31.62 & 0.1652 & 61.25 \\
& 50 & 30.80 & 0.1413 & 62.30 \\
& 55 & 30.15 & 0.1218 & 62.90 \\
& 60 & 29.61 & 0.1057 & 63.21 \\
\hline ISD-112\&112 & Temp- ${ }^{\circ} \mathrm{C}$ & Freq-rad/s & Loss-factor & SPL-dB \\
\hline \hline & 40 & 32.88 & 0.4427 & 54.31 \\
& 45 & 30.76 & 0.3506 & 56.45 \\
& 50 & 29.30 & 0.2776 & 57.68 \\
& 55 & 28.22 & 0.2252 & 58.05 \\
& 60 & 27.54 & 0.1850 & 59.67 \\
\hline
\end{tabular}

The relationship between the loss factor and the peak value of SPL can be investigated through the trends found in Table 6.11 .

For the same damping material, as the ambient temperature is increased, the peak value of SPL increases while the loss factor decreases.

For the different damping materials with the same temperature, the peak value of SPL is higher with the lower loss factor. 


\section{Conclusions and Recommendations}

\subsection{Conclusions}

A framework of FE-based vibro-acoustical analysis for a multiple-layer beam structure is presented by introducing different types of viscoelastic materials. Several conclusions for each step can be drawn in this research:

1. The FE model of a multiple-layered damping beam is established by proper coordinate transformation of two fundamental elements: elastic layer and constrained damping layer. This methodology can be extended from the sandwich beam to more complicated damping structures without much effort.

2. The non-linear curve-fitting technique accurately models the Biot constants for further vibration analysis. Results from the vibration analysis procedure discussed in this research are comparible with the closed form solution from previous work and simulations from ANSYS result. The Biot damping model is also capable of improving the structure's damping performance by adding new features such as: different viscoelastic materials, the variation of ambient 
temperature, etc.

3. Decoupling transformation not only solves the eigenvalue-based vibration problem to explain the time-domain response of energy dissipation, but also successfully establishes the FRF-based frequency-spectrum analysis. The first peak from the frequency-spectrum is the dominant cause of the vibration issue in this damping structure.

4. The Direct Boundary Element Method (DBEM) analysis for acoustical cavity with anechoic boundaries is used to solve the acoustical problem by using the particle velocity result from frequency-spectrum analysis. The acoustical result matches the frequency-spectrum result from vibration analysis as well as simulation results. It also shows the relationship between loss factor and sound pressure level (SPL) of the first dominant peak. 


\subsection{Recommendation for Future Research}

1. Curve fitting

Build more advanced Biot curve-fitting techniques of complex shear modulus with more robustness and efficiency in order to apply to a wider temperature range.

2. Higher order damping model

As discussed in the damping mechanism section, Biot can be seen as the first-order of GHM damping model. The Biot damping model has less accuracy with the same order of damping constants in the curve-fitting process compared to a second-order mini-oscillator damping model. Thus the second-order mini-oscillator model can be used for a wider temperature range. It would be interesting to investigate and develop the other dynamic equations with GHM or similar damping models [108].

3. Optimization 
Determine an optimization algorithm on the basis of vibro-acoustical responses;

particularly maximize the loss while trying to minimize the peak value of sound

pressure by determining the optimal parameters such as number of layers, sizing, placement, etc.

4. Application to Complicated/3D structures

Extend the FE model from a simple beam structure to an irregular shape structure $[38,109,110]$ for the industrial usage. For a 3D geometry, the Biot damping model requires further manipulation to incorporate a more complicated form of Biot constants. This is because viscoelastic material behaves in a non linear manner in 3D situations, and the complex shear modulus needs to be considered as having multi-directional parameters.

\section{Testing}

Fabricate multiple-layer sandwich damping beams with different damping materials and design the vibro-acoustical experimentation to validate the simulation results. From the $2 \mathrm{D}$ BEM result, the most challenging task is the measurement of low-frequency sound signal. It is also needed to lower the 
background noise due to the exceptional acoustical-attenuation performance of

high-damping structures.

\section{Control}

ACL (Active Control Layer) treatment [64, 111-113] is still an active research in recent years. It is necessary to develop the control strategy for a multiple-layer damping structure and investigate the relationship between control parameters and damping factors as well as vibro-acoustical responses. 


\section{Bibliography}

1 Lienard, P. Etude d'une Methode de Mesure du Frottement Interieur de Revetements Plastiques Travaillant en Flexion. La Recherche Aeronautique, 1951, 20, $11-22$.

2 Oberst, H., and Frankenfeld, K. Uber Die Dampfung der Biegeschwingungen Dunner Bleche Durch Fest Haftende Belage I. Acustica, 1952, 2, 181-194.

3 Oberst, H., Becker, G. W., and Frankenfeld, K. Uber Die Dampfung Der Biegeschwingungen Dunner Bleche Durch Fest Haftende Belage II. Acustica, 1954, $4,433-444$.

4 Oberst, H. Werkstoffe Mit Extrem Hoher Innerer Dampfung. Acustica(Akustiche Beihefte), 1956, 6, 144-153.

5 Plass, H.J. Damping of Vibrations in Elastic Rods and Sandwich Structures by Incorporation of Additional Viscoelastic Material. In 3rd Midwestern Conference on Solid Mechanics, 1957, 48-71.

6 Kerwin, E.M. Damping of Flexural Waves by a Constrained Visco-Elastic Layer. Journal of the Acoustical Society of America, 1959, 31, 952-962.

7 Kerwin, E.M., and Ross, D. A Comparison of the Effectiveness of 
Homogeneous Layers and Constrained Layers of Viscoelastic Material in Damping

Flexural Waves in Plates. 3rd International Congress on Acoustics, 1959, 410-412.

8 Ross, D., Ungar, E. E., and Kerwin, E. M. Damping of Plate Flexural

Vibration by means of Viscoelastic Laminae. Structural Damping-a colloquium on structural damping held at the ASME annual meeting, 1959, 49-87.

9 Ditaranto, R.A. Thery of Vibratory Bending for Elastic and Viscoelastic Layered Finite-Length Beams. Journal of Applied Mechanics, 1965, 32, 881-886.

10 Mead, D.J., and Markus, S. The Forced Vibration of a Three-Layer, Damped Sandwich Beam with Arbitrary Boundary Conditions. Journal of Sound and Vibration, 1969, 10(2), 163-175.

11 Rao, M.D. Recent Applications of Viscoelastic Damping for Noise Control in Automobiles and Commercial Airplanes. Journal of Sound and Vibration, 2003, 262(3), 457-474.

12 Cottle, E.T. Damping of Layered Beams with Mixed Boundary Conditions. Master Thesis, Air Force Institute of Technology, Dec, 1990.

13 Torvik, P.J. The Analysis and Design of Constrained Layer Damping Treatments. In Proceedings ASME Winter Annual Meeting - Damping Applications for Vibration Control, pp. 85-112 (ASME, 1980).

14 Mead, D.J. A Comparison of Some Equations for the Flexural Vibration of 
Damped Sandwich Beams. Journal of Sound and Vibration, 1982, 83(3), 363-377.

15 Nakra, B.C. Vibration Control with Viscoelastic Materials. Shock and Vibration Digest, 1976, 8(6), 3-12.

16 Nakra, B.C. Vibration Control with Viscoelastic Materials II. Shock and Vibration Digest, 1981, 13(1), 17-20.

17 Nakra, B.C. Vibration Control with Viscoelastic Materials III. Shock and Vibration Digest, 1984, 16(5), 17-22.

18 Douglas, B.E., and Yang, J. C. S. Transverse Compressional Damping in the Vibratory Response of Elastic-Viscoelastic-Elastic Beams. AIAA Journal, 1978, 16(9), 925-930.

19 Ma, B.A. Loss Factor and Natural Frequency of Five-Layer Viscoelastic Damping Beam. Journal of Mechanics in Engineering, 1982, 1, 39-43.

20 Hao, M., and Rao, M. D. Vibration and Damping Analysis of a Sandwich Beam Containing a Viscoelastic Constraining Layer. Journal of Composite Materials, 2005, 39(18).

21 Teng, T., and Hu, N. K. Analysis Of Damping Characteristics For Viscoelastic Laminated Beams. Comput. Methods Appl. Mech. Engrg., 2001, 190, 3881-3892.

22 Falugi, M. Analysis of a Five-Layer, Viscoelastic, Constrained-Layer Beam. Proceedings of Damping Workshop Paper, 1991, CCB. 
23 Parin, M., Rogers, L. C., and Falugi, M. Practical Stand Off Damping Treatment for Sheet Metal. Proceedings of Damping Workshop Paper, 1989, IBA.

24 Rogers, L.C., and Parin, M. Experimental Results for Stand-off passive vibration damping treatment. Proceedings of SPIE- the International Society for Optical Engineering, 1995, 2445, 374-383.

25 Yellin, J.M., Shen, I. Y., and Reinhall, P. G. An Experimental Investigation of a Passive Stand-Off Layer Damping Treatment Applied to an Euler-Bernoulli Beam. Proceedings of SPIE, 1999, 3672, 228-233.

26 Yellin, J.M., Shen, I. Y., and Reinhall, P. G. An Analytical and Experimental Analysis for a One-Dimensional Passive Stand-Off Layer Damping Treatment. Transactions of ASME, Oct, 2000, 122, 440-447.

27 Yellin, J.M., and S., I. Y. An Analytical Model for a Passive Stand-Off Layer Damping Treatment Applied to an Euler-Bernoulli Beam. Proceedings of SPIE, 1998, 3327, 349-356.

28 Yang, B., and T., C. A. The Transfer Functions of One Dimensional Distributed Parameter Systems. Journal of Applied Mechanics, 1959, 116, 341-349.

29 Vibration Damping 1984 Workshop Proceeding. (Air Force Wright Aeronautical Laboratories, 1984).

30 Damping 1986 Proceedings. (Air Force Wright Aeronautical Laboratories, 
1986).

31 Proceedings of Damping '89. (Air Force Wright Aeronautical Laboratories, 1989).

32 Proceedings of Damping '91. (Air Force Wright Aeronautical Laboratories, 1991).

33 Proceedings of Damping '93. (Air Force Wright Aeronautical Laboratories, 1993).

34 Johnson, C.D., Kienholz, D. A., and Rogers, L. C. Finite Element Prediction of Damping in Beams with Constrained Viscoelastic Layers. The Shock and Vibration Bulletin, 1981, 51(1), 71-81.

35 Ungar, E.E., and Kerwin, E. M. Loss Factors of Viscoelastic Systems in terms of Energy Concepts. Journal of the Acoustical Society of America, 1962, 34, 954-957.

36 Lesieutre, G.A., and Lee, U. A Finite Element For Beams Having Segmented Active Constrained Layers With Frequency. Smart Material Structure, 1996, 5, 615-627.

37 Chen, Q., and Chan, Y. W. Integral Finite Element Method for Dynamical Analysis of Elastic-Viscoelastic Composite Structures. Computers and Structures, 2000, 72, 51-64. 
38 Kung, S.W., and Singh, R. Development Of Approximate Methods For The Analysis Of Patch Damping. Journal of Sound and Vibration, 1999, 219(5), 785-812.

39 Zhang, Q.J., and Sainsbury, M. G. The Galerkin Element Method Applied to The Vibration of Rectangular Damped. Computers and Structures, 2000, 74, 717-730.

40 Zhang, Y., Zhang, Z. Y., Tong, Z. P. and Hua, H. X. Finite Element Modeling of A Fluid Filled Cylindrical Shell. Journal of Shanghai Jiaotong University(Science), 2005, E10(4), 449-455.

41 Balmes, E., and Germes,S. Tools for Viscoelastic Damping Treatment Design, Application to An Automotive Floor Panel. ISMA, 2002.

42 SIMULIA. ABAQUS 6.7 Online Documentation. Dassault Systèmes, 2007.

43 Mayergoyz, I.D. Mathematical Models of Hysteresis IEEE Transactions on Magnetics, 1986.

44 Soussou, J.E., Moavenzadeh, F., Gradowczyk, M. H. Application of Prony Series to Linear Viscoelasticity. Transactions of the Society of Rheology, 1970, 14(4), 573-584.

45 Bagley, R.L., and Torvik, P. J. Fractional Calculus-a Different Approach To The Analysis of Viscoelastically Damped Structures. AIAA Journal, 1983, 21(5). 
46 Lesieutre, G.A., and Mingori, D. L. Finite Element Modeling of

Frequency-dependent Material Damping Using Augmenting Thermodynamic Fields.

Journal of Guidance Control and Dynamics, 1990, 13(6), 1040-1050.

47 Lesieutre, G.A., and Bianchini, E. Finite Element Method of One-Dimensional

Viscoelastic Structures Using Anelastic Displacement Fields. Journal of Guidance

Control and Dynamics, 1996, 19(3).

48 Biot, M.A. Variational Principles in Irreversible Thermodynamic with

Application to Viscoelasticity. Physical Review, 1955, 97(6), 1463-1469.

49 Golla, D., and H., P. Dynamics of Viscoelastic Structures - A Time Domain Finite Element Formulation. Journal of Applied Mechanics, 1985, 52, 897-906.

50 McTavish, D.J. Shock Response of a Damped Linear Structure Using GHM Finite Element. AIAA Journal, 2003, 1591.

51 Yiu, Y.C. Finite Element Analysis of Structures with Classical Viscoelastic Materials. in 34th AIAA/ASME/ASCE/AHS/ASC Structures, Structural Dynamics, and Materials Conference, 1993(2110-2119).

52 Adhikari, S. Damping Models for Structural Vibration. Cambridge University Engineering Department (September,2000).

53 Zhang, J., and Zheng, G. T. The Biot Model and Its Application in Viscoelastic Composite Structures. Journal of Vibration and Acoustics, 2007, 129, 533-540. 
54 Zhang, L., Du, H. P., Shi, Y. M. and Shi, X. Z. Parametric Determination for GHM of ZN-1 Viscoelastic Material. Raremetal Materials and Engineering, 2002, 31(2), 92-95.

55 Park, C.H., Inman, D. J., and Lam, M. J. Model Reduction of Viscoelastic Finite Element Models. Journal of Sound and Vibration, 1999, 219(4), 619-637.

56 Plump, J.M., and Hubbard Jr., J.E. Modeling of an Active Constrained Layer Damper. in 12th International Congress on Acoustics, Toronto, Canada, 1986.

57 Lagnese, T.J., and Jones, D. I. G. Complex Modulus Behavior of a Viscoelastic Adhesive Measured at Harmonic Strain Amplitudes of $10^{-10}$. In Vibration Damping 1984 Workshop Proceedings, pp. E1-E17, Long Beach, CA, (1984).

58 Austin, E.M., Flora, T., and Gooding, J. Low-Deflection Loss and Hysteresis Measurements on a Spacecraft Test Joint. In Damping '91 (San Diego, CA), 1991, III, IBC1-IBC19.

59 Liao, W.H., and Wang, K. W. On the Active-Passive Hybrid Control of Structures with Active Constrained Layer Treatments. Journal of Vibration and Acoustics, 1997, 119(4), 563-572.

60 Shen, I.Y. Hybrid Damping Through Intelligent Constrained Layer Treatments. Journal of Vibration and Acoustics, 1994, 116(3), 341-349. 
61 Shen, I.Y. Stability and Controllability of Euler-Bernoulli Beams with Intelligent Constrained Layer Treatments. Journal of Vibration and Acoustics, 1996, $118(1), 70-77$.

62 Varadan, V.V., Lim, Y.H., and Varadan, V.K. Closed Loop Finite-Element Modeling of Active/Passive Damping in Structural Vibration Control. Smart Materials and Structures, 1996, 5(5), 685-694.

63 Baz, A. Optimization of Energy Dissipation Characteristics of Active Constrained Layer Damping. Smart Materials and Structures, 1997, 6(3), 360-368.

64 Trindade, M.A., Benjeddou,A., and Ohayon, R. Modeling of Frequency-dependent Viscoelastic Materials for Active-passive Vibration. Journal of Vibration and Acoustics, 2000, 112, 169-174.

65 Agnes, G.S., and N., K. Active Constrained Layer Viscoelastic Damping. in 34th AIAA/ASME/ASCE/AHS/ASC Structures, Structural Dynamics, and Materials Conference, AIAA, 1993, 3499-3506.

66 Yellin, J.M, and S., I.Y. A Self-Sensing Active Constrained Layer Damping Treatment for a Euler-Bernoulli Beam. 1996.

67 Huang, S.C., Inman, D.J., and Austin, E.M. Some Design Considerations for Active and Passive Constrained Layer Damping Treatments. Smart Materials and Structures, 1996, 5(3), 301-313. 
68 Dosch, J.J., Inman, D.J., and Garcia, E. A Self-Sensing Piezoelectric Actuator for Collocated Control. Journal of Intelligent Material Systems and Structures, 1992, 3(1), 167-185.

69 Baz, A., and Ro, J. Optimum Design and Control of Active Constrained Layer Damping. JOurnal of Mechanical Design and Journal of Vibration and Acoustics, 1995, 117(B), 135-144.

70 Baz, A., and R., J. Partial Treatment of Flexible Beams with Active Constrained Layer Damping. in Recent Developments in Stability, Vibration and Control of Structural Systems, 1993, AMD(167), 61-80.

71 Baz, A. Robust Control of Active Constrained Layer Damping. Journal of Sound and Vibration, 1998, 211(3), 467-480.

72 Trindade, M.A., and Benjeddou, A. Hybrid Active-Passive Damping Treatments Using Visco-elastic and Piezoelectric Materials. Journal of Vibration and Control, 2002, 8, 699-745.

73 Kim, S.Y., and Lee, D. H. Identification of Fractional-Derivative Model Parameters of Viscoelastic materials from measured FRFs Journal of Sound and Vibration, 2009, 325(3-5), 570-586.

74 Plunkett, R., and Lee, C. T. Length Optimization for Constrained Viscoelastic Layer Damping. Journal of the Acoustical Society of America, 1970, 48(1), 150-161. 
75 Lall, A.K., Nakra, B. C., and Asnani, N. T. Optimum Design of Viscoelastically Damped Sandwich Panels. Engineering Optimization, 1983, 6, 197-205.

76 Lifshitz, J.M., and Leibowitz, M. Optimal Sandwich Beam Design for Maximum Viscoelastic Damping. International Journal of Solids and Structures, 1987, 23(7), 1027-1034.

77 Marcelin, J.L., Trompette, P., and Smatic, A. Optimal Constrained Layer Damping with Partial Coverage. Finite Elements in Analysis and Design, 1992, 12, 273-280.

78 Baz, A., and Ro, J. Optimum Design and Control of Active Constrained Layer Damping. Journal of Mechanical Design, 1995, 117(Special Issue), 135-144.

79 Nakra, B.C. Vibration Control in Machines and Structures Using Viscoelastic Damping. Journal of Sound and Vibration, 1998, 211(3), 449-465.

80 Zheng, H., Cai, C., and Tan, X. M. Optimization of Partial Constrained Layer Damping Treatment for Vibrational Energy Minimization of Vibrating Beams. Computers and Structures, 2004, 82, 2493-2507.

81 Kim, T.W., and Kim, J. H. Eigensensitivity Based Optimal Distribution of a Viscoelastic Damping Layer for a Flexible Beam. Journal of Sound and Vibration, 2004, 273, 201-218. 
82 Lee, D.H., and Hwang, W. S. Layout Optimization of Unconstrained Viscoelastic Layer on Beams Using Fractional Derivative Model. AIAA Journal, 2004, 42(10), 2167-2170.

83 Kruger, D.H., and Mann, J. A. Minimizing the Sound Power Radiated by a Cube as a Function of the Size of Constrained Layer Damping Patches. Journal of the Acoustical Society of America, 1999, 105(3), 1714-1724.

84 Azzouz, M.S., and Ro, J. Control of Sound Radiation of an Active Constrained Layer Damping Plate/Cavity System Using the Structural Intensity Approach. Journal of Vibration and Control, 2002, 8(6), 903-918.

85 Gibbs, G.P., and Cabell, R. H. Active/Passive Control of Sound Radiation from Panels Using Constrained Layer Damping. AIAA Journal, 2003, 1813.

86 Zheng, H., and Cai, C. Minimization of Sound Radiation from Baffled Beams Through Optimization of Partial Constrained Layer Damping Treatment. Applied Acoustics, 2004, 65, 501-520.

87 Hao, M., and Rao, M. D. Optimum Design of Multiple-Constraint Layered Systems for Vibration Control. AIAA Journal, 2004, 42(12), 2448-2461.

88 Lee, D.H. Optimal Placement of a Constrained-layer Damping For Reduction of Interior Noise. AIAA Journal, 2008, 42(12), 75-83.

89 Drozdov, A.D. Mechanics of Viscoelastic Solids. John Wiley \& Sons, 1998, 
0-471-97512.

90 Chandrupatla, T.R., and Belequndu, A. D. Introduction to Finite Elements in Engineering. Prentice Hall, 2002, 3rd Edition.

91 Trindade, M.A., and Benjeddou, A. Reduced-Order Finite Element Models of Viscoelastically Damped Beams through Internal Variables Projection. Journal of Vibration and Acoustics, 2006, 128(4), 501-508.

92 Lam, M.J., Inman, D. J. and Saunders, W. R. Hybrid Damping Models Using The Golla-Hughes-McTavish Method With Internally Balanced Model Reduction And Output Feedback. Smart Material Structure, 2000, 9, 362-371.

93 3M. Product Dcoumentation of $3 \mathrm{M}^{\mathrm{TM}}$ Viscoelastic Damping Polymers (online).

94 Hao, M. Vibration Analysis of Constrained Layered Beams with Multiple Damping Layers. Michigan Tech, 2005, Ph.D Dissertation.

95 Chen, L.H., and S., D. G. Sound Radiation From an Arbitrary Body. The Journal of Acoustical Society of America, 1963, 35, 1626-1632.

96 Chertock, G. Sound Radiation from Vibrating Surfaces. The Journal of Acoustical Society of America, 1964, 36, 1305-1313.

97 Copley, L.G. Integral Equation Method for Radiation from Vibrating Bodies. The Journal of Acoustical Society of America, 1967, 41, 807-816.

98 Schenck, H.A. Improved Integral Formulation for Acoustic Radiation Problem. 
The Journal of Acoustical Society of America, 1968, 44, 41-58.

99 Waterman, P.C. New Formulation of Acoustic Scattering. The Journal of Acoustical Society of America, 1969, 45, 1417-1429.

100 Burton, A.J., and Miller, G. F. The Application of Integral Equation Method on the Numerical Solutions of Some Exterior Boundary Value Problem. Proceedings of Royal Society of London, 1971, A323, 201-210.

101 Ursell, F. On the Exterior Problems of Acoustics. Proceedings of Cambridge Philosophy Society, 1973, 74, 117-125.

102 Hamdi, M.A. Une Formulation Variationnelle par Equations Integrales Pour le Resolution de l'equation de Helmholtz avec des Conditions aux Limites Mixtes. Comtes Rendus Acad. Sc. Paris, 1981, 292(Ser. II), 17-20.

103 Mariem, J.B.and H., M. A. A New Boundary Finite Element Method for Fluid-Structure Interaction Problems. International Journal of Numerical Methods in Engineering, 1987, 24, 1251-1267.

104 Wu, X., F., Pierce, A. D. and Ginsberg, J. H. Variational Method for Computing Surface Acoustic Pressure on Vibrating Bodies, Applied to Transversely Oscillating Disks. IEEE Journal of Ocenic Engineering, 1987, OE(12), 412-418. 105 ANSYS. Dynamics Module 3-Harmonic Dynamic. ANSYS Standard Training, 2005. 
106 Hong, K. LMS Virtual.Lab BEM Acoustics-Valve Cover. LMS Virtual.Lab Training, 2006.

107 Phillips, C.L., and Harbor, R. D. Feedback Control Systems (4th Edition) (Prentice Hall, 1999).

108 Johnson, A.R. Modeling Viscoelastic Material Using Internal Variables. The Shock and Vibration Digest, 1999, 31(91).

109 Wang, G., Veeramani, S. and Wereley, N. M. Analysis Of Sandwich Plates With Isotropic Face Plates And A Viscoelastic Core. Journal of Vibration and Acoustics, 2000, 122, 305-312.

110 Hammaml, L., Zghal, B., Fakhfakh, T., and Haddar, M. Characterization Of Modal Damping Of Sandwich Plates. Journal of Vibration and Acoustics, 2005, 127, 431-440.

111 Liao, W.H., and Wang, K. W. On The Analysis of Viscoelastic Materials For Active Constrained Layer Damping Treatments. Journal of Sound and Vibration, 1997, 207(3), 319-334.

112 Xie, S.L., Zhang, X. N., Zhang,J. H., and Yu, L. Robust Vibration Control of a Thin Plate Covered With a Controllable Constraned Damping. Journal of Vibration and Control, 2004, 10(115).

113 Balamurugan, V. Finite Element Formulation And Active Vibration Control 
Study On Beams Using Smart Control. Journal of Sound and Vibration, 2002, 249(2), 227-250. 


\section{Appendix A-Note of Matlab Code}

(The code can be acquired from author per request)

\begin{tabular}{|c|c|}
\hline Filename & Explanation \\
\hline Mainentry_1type.m & Main program, calculating loss/freq for $1 \mathrm{vem}$ \\
\hline Mainentry_2type.m & Main program, calculating loss/freq for $2 \mathrm{vems}$ \\
\hline Mainentry_comparefreq.m & Main program, plotting loss/freq versus temp \\
\hline Mainentry_2type_acoust.m & Main program, plotting SPL versus temp \\
\hline FE_7layer.m & Subroutine, FE mode-seven layers beam \\
\hline FE_7layer_2type.m & Subroutine, FE mode-seven layers beam with 2 VEMs \\
\hline Combodel.m & Function of FE_7layer;helping apply the BC \\
\hline Delrowcol.m & Function of FE_7layer \\
\hline Biotpara.m & Subroutine, Provide Biot parameters \\
\hline Biotmodel1_zeroeig_cal.m & Subroutine, construct the Biot dynamic equation for 1vem \\
\hline Biotmodel2_zeroeig_cal.m & Subroutine, construct the Biot dynamic equation for 2 vems \\
\hline Decoupling.m & Subroutine, implement decoupling transformation \\
\hline Noise.m & Subroutine, interface with Fortran Program-DBEM2D.exe \\
\hline Writetxt.m & Function of noise.m, reading txt to workspace \\
\hline Readtxt.m & Function of noise.m, reading workspace to txt \\
\hline ISD3M.m & Original complex shear modulus based on Arrhenius Equation \\
\hline Finallumda.m & Function, calculating loss factor \\
\hline
\end{tabular}




\section{Appendix B-Curve fitting Datasheet for 3M-ISD110/112}

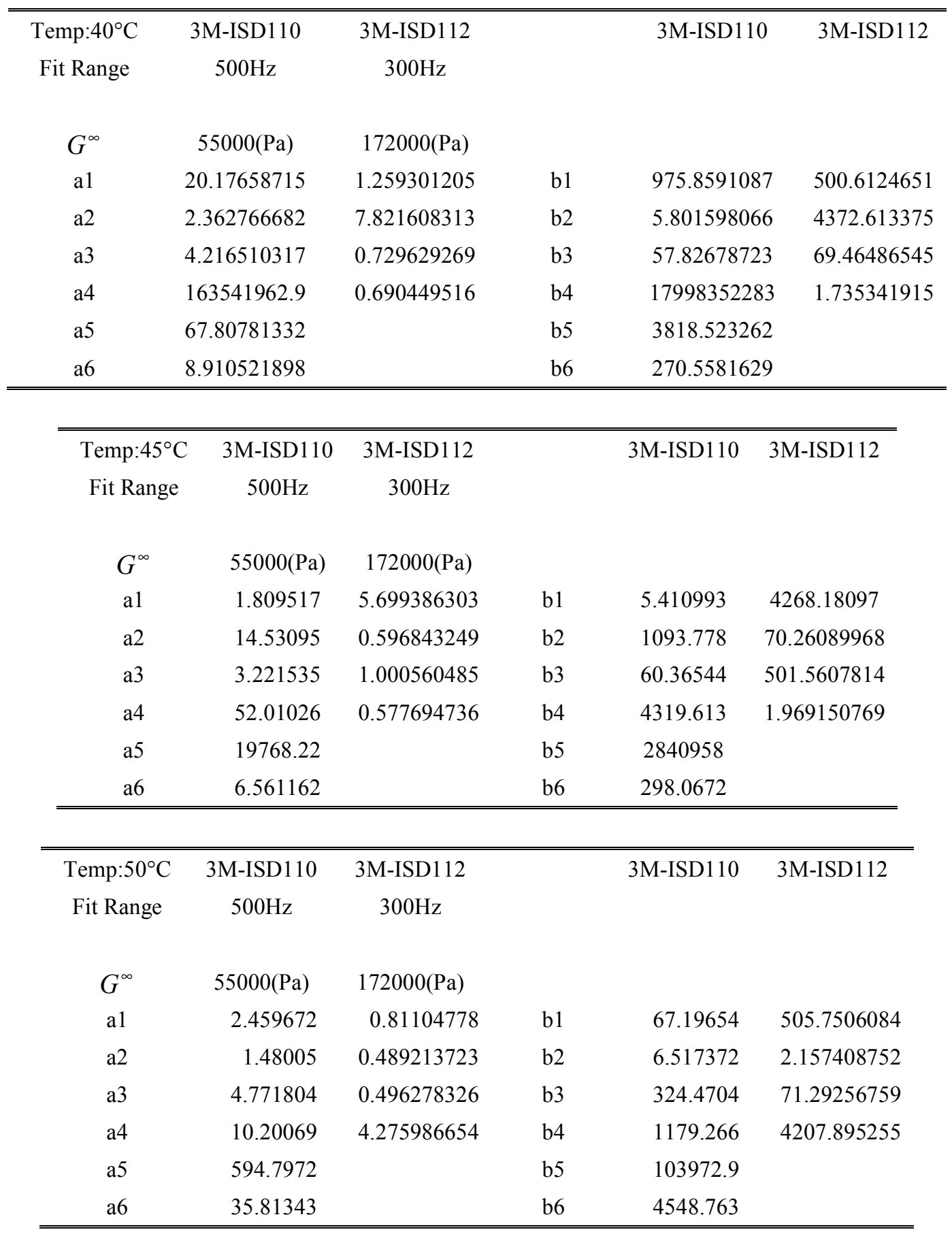




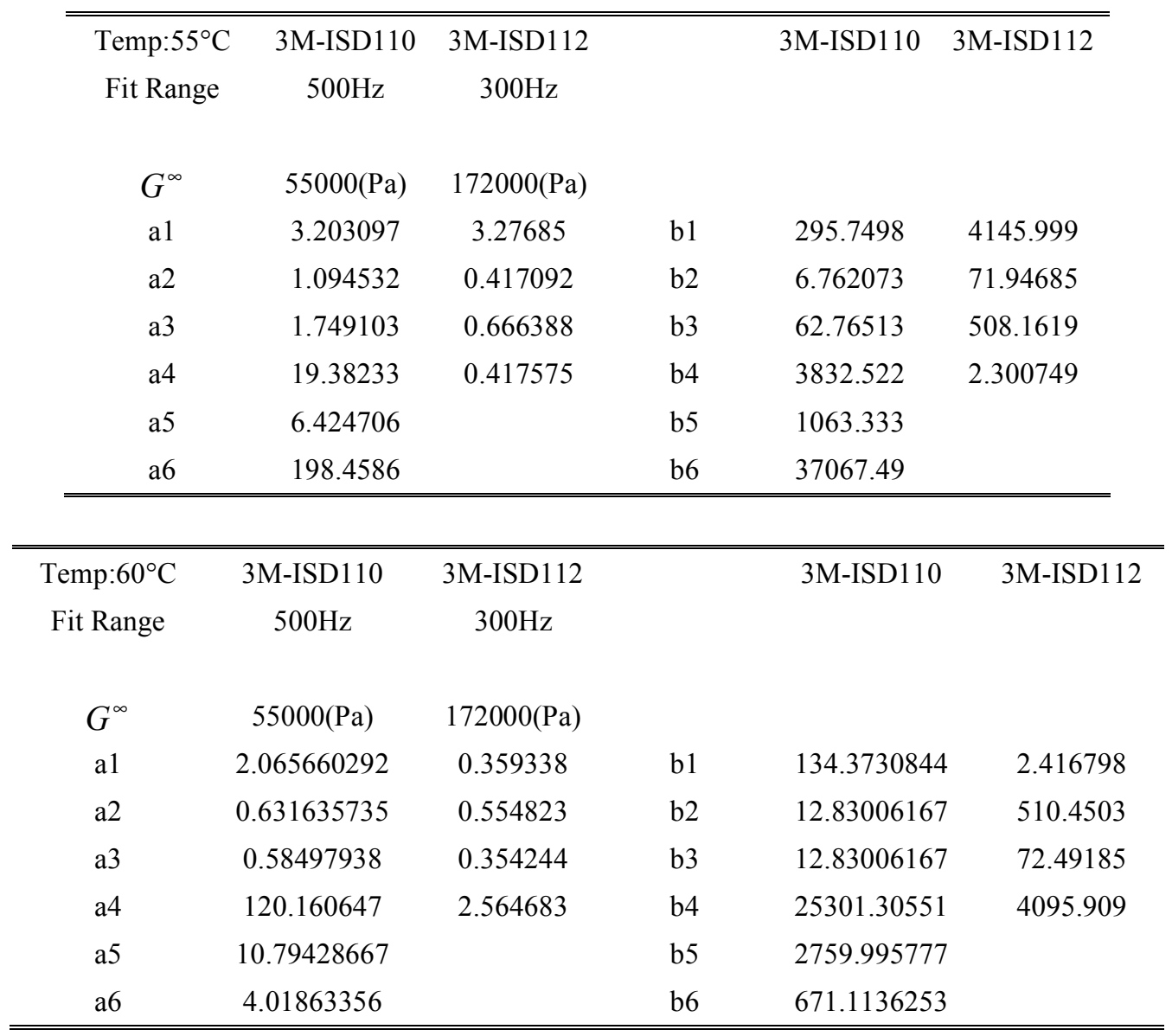




\section{Appendix C- Hankel Function}

Recalling $\boldsymbol{\Psi}=-\frac{i}{4} \mathbf{H}_{0}^{(2)}(k r)$ as the general solution of Helmholtz equation (5.1), Henkel function $\left(2^{\text {nd }}\right.$ kind $)$ are defined as:

$$
\mathbf{H}_{\alpha}^{(2)}(x)=J_{\alpha}(x)-i Y_{\alpha}(x)
$$

Where $J_{\alpha}(x)$ is the Bessel function of first kind and $Y_{\alpha}(x)$ is the Bessel function of second kind. Practically, the Hankel function of second kind can be implemented as:

$$
\mathbf{H}_{\alpha}^{(2)}(x)=\frac{J_{-\alpha}(x)-e^{-\alpha \pi i} J_{\alpha}(x)}{-i \sin (\alpha \pi)}
$$

Due to $\alpha$ is equal to zero in above equation causing the denominator equal to zero, the limit value is necessary to be calculated for equation on RHS.

$J_{\alpha}(x)$-Bessel function of first kind- can be represented with an integral form when alpha is the integral number:

$$
J_{\alpha}(x)=\frac{1}{2 \pi} \int_{o}^{2 \pi} \cos (\alpha \tau-x \sin \tau) d \tau=\frac{1}{2 \pi} \int_{-\pi}^{\pi} e^{\alpha \tau-x \sin \tau} d \tau
$$

